\title{
TEACHER COLLABORATION AND INSTRUCTIONAL PROGRAM COHERENCE ACROSS THE TRANSITION TO HIGH SCHOOL
}

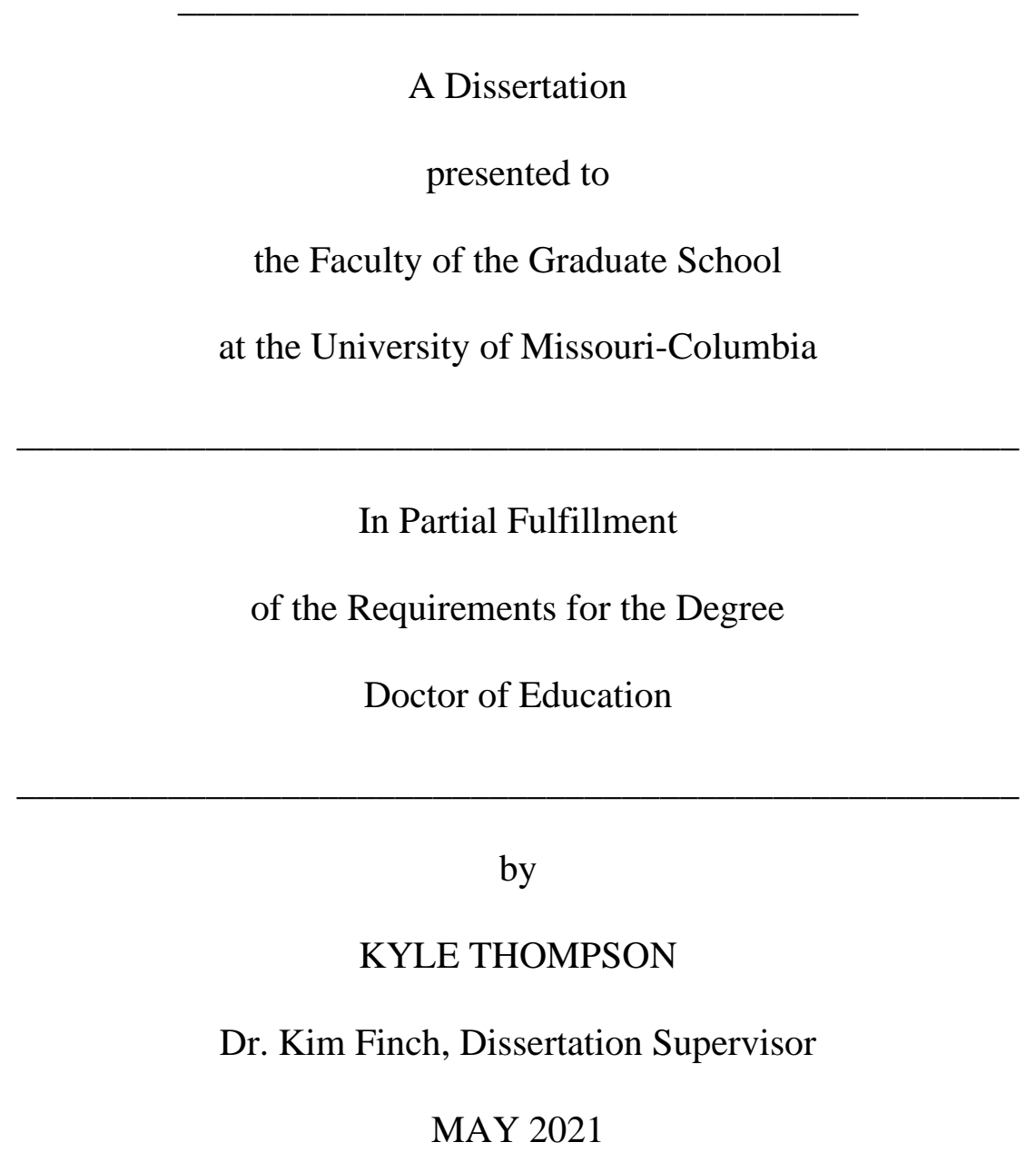


The undersigned, appointed by the dean of the Graduate School, have examined the dissertation entitled

\section{TEACHER COLLABORATION AND INSTRUCTIONAL PROGRAM COHERENCE ACROSS THE TRANSITION TO HIGH SCHOOL}

presented by Kyle Thompson

a candidate for the degree of doctor of educational leadership and policy analysis, and hereby certify that, in their opinion, it is worthy of acceptance.

\begin{tabular}{c}
\hline Dr. Kim Finch \\
\hline Dr. Cynthia MacGregor \\
\hline Dr. Kennedy Ongaga \\
\hline
\end{tabular}

Dr. William Agnew 


\section{DEDICATION}

I dedicate my dissertation work to my parents and my heroes, James and Mary Chris Thompson for being my example and showing me the true meaning of selflessness. You risked so much to always put the needs of us kids before your own. Everything good in my life I attribute to you and I am truly fortunate. I could not have and more importantly would not have done any of this without you.

I also dedicate this to Ma and $\mathrm{Pa}$. Your inspiration both professionally and personally led me on this path. You have been with me throughout, more than you realize.

To Dr. Julie and Dr. Mike, thank you for being the best two summer friends a person could ask for.

To Sonya Deterding, the best teacher I have ever had the honor of working beside. Without your help as a colleague, but more importantly, a friend, this would not have been possible. Thank you.

To Dr. Allen Grymes, from the bottom of my heart thank you for everything.

Finally, to my entire family, I also dedicate this dissertation to you. We have sacrificed valuable time together so I could pursue this goal and you have been nothing but patient and supportive. I have watched from afar and have been so proud to see how our family has grown. I miss you and love you all. To Alicia, there was never a shadow cast as you have always been a source of sunlight that I will forever be thankful has been shining in my life since the beginning. 


\section{ACKNOWLEDGEMENTS}

A heartfelt thank you must first go to Dr. Finch. In a time of great adversity, you have been unbelievably selfless. You were a constant voice of support and knowledge that kept me moving forward when I didn't think I could. I honestly do not know how you did it all. You are truly an amazing person.

Thank you to my committee members Dr. Macgregor, Dr. Ongaga, and Dr. Agnew for

helping me through this process. I'd also like to thank Dr. Macgregor and Dr. Ongaga for being two of the best educators I have had the good fortune of learning from.

To my cohort, you were and always will be family. Thanks for making this journey one of the most amazing experiences I have ever had and all the help along the way. 


\section{TABLE OF CONTENTS}

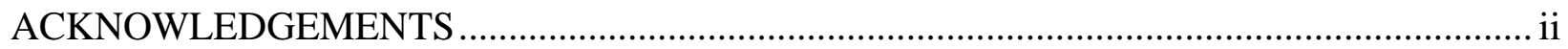

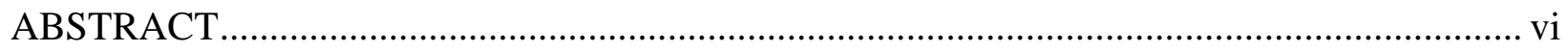

SECTION ONE: INTRODUCTION TO DISSERTATION ................................................. 1

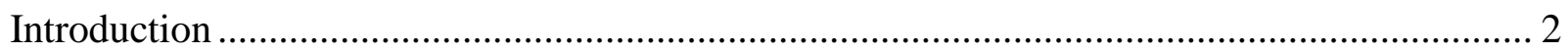

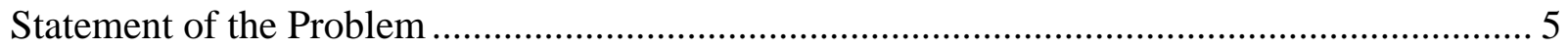

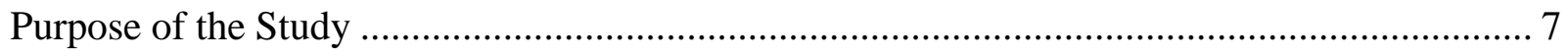

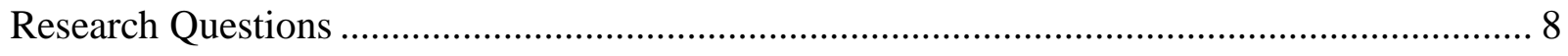

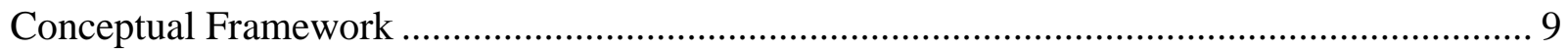

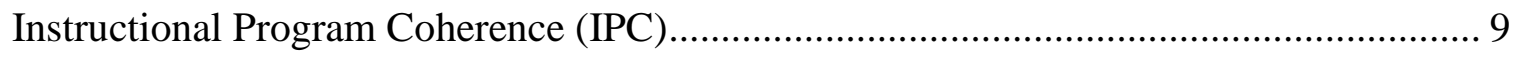

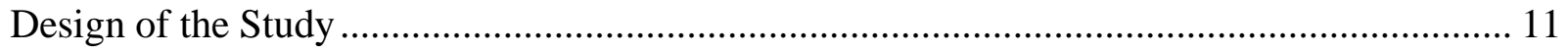

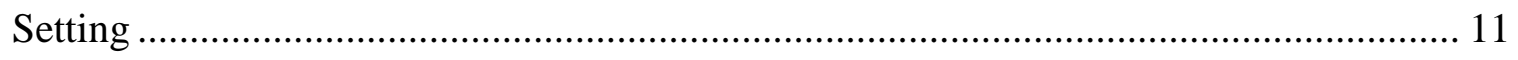

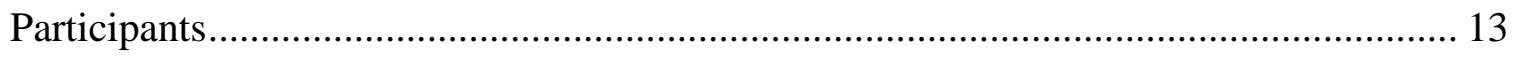

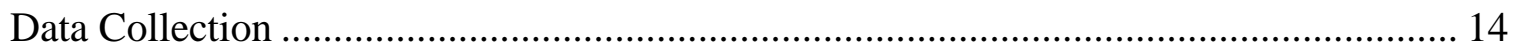

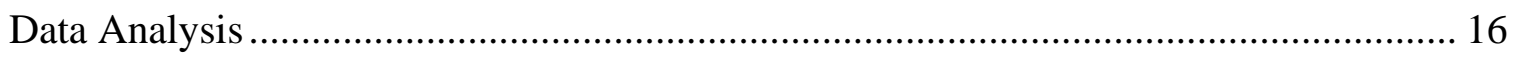

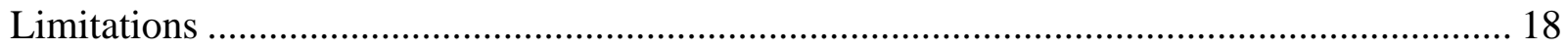

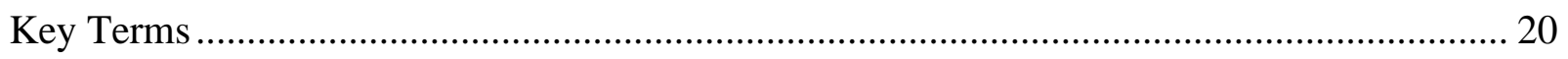

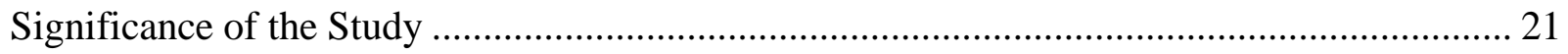

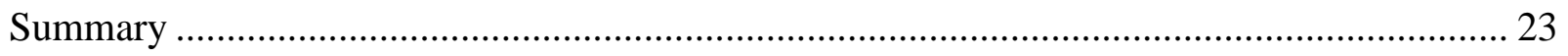

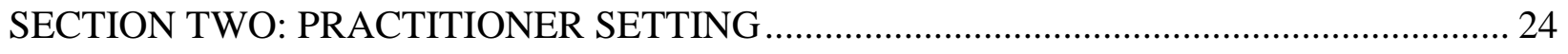

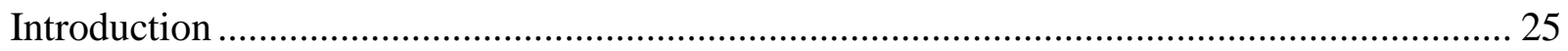

History and Background of the Organization ............................................................. 25

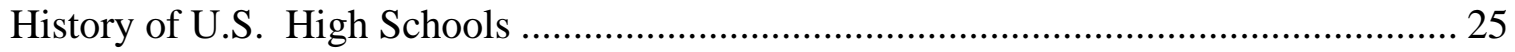


The State of Missouri Educational Structure ................................................................... 30

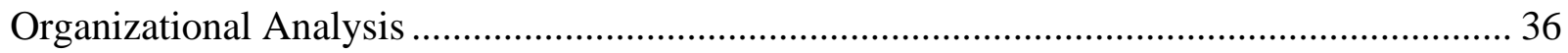

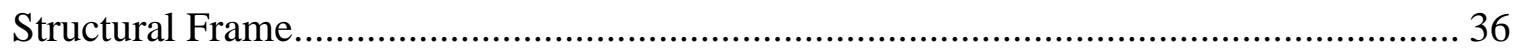

Leadership Analysis ........................................................................................................ 43

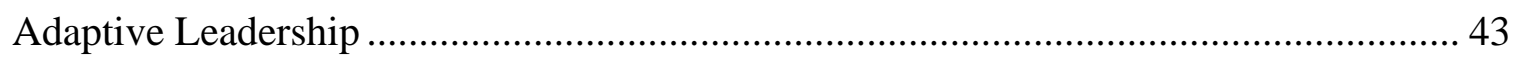

Implications for Research in the Practitioner Setting............................................................... 48

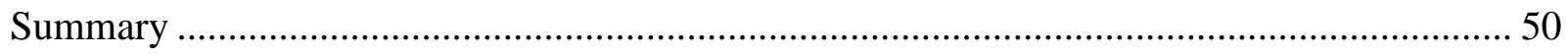

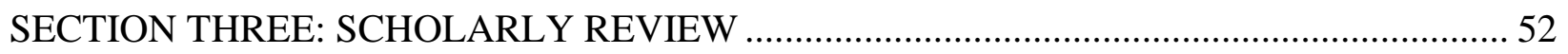

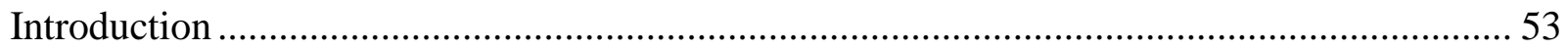

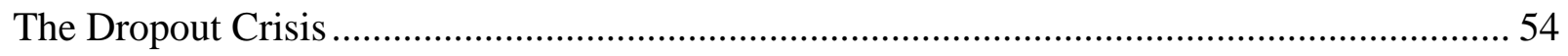

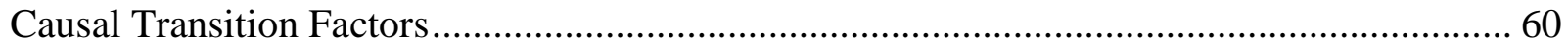

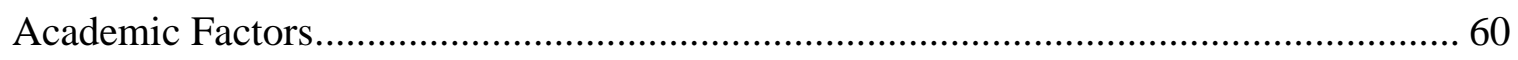

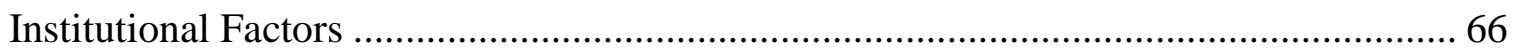

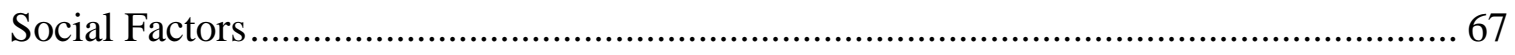

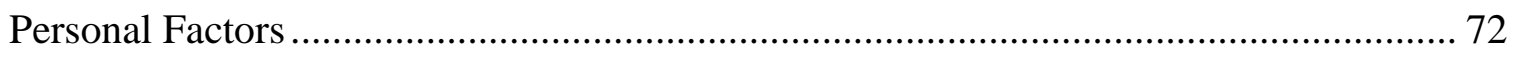

Consequences of Transitional Factors............................................................................... 74

Psychological Consequences …………………………........................................... 75

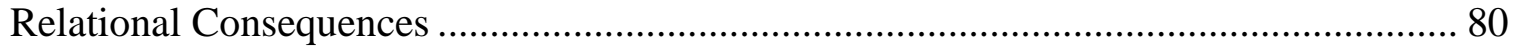

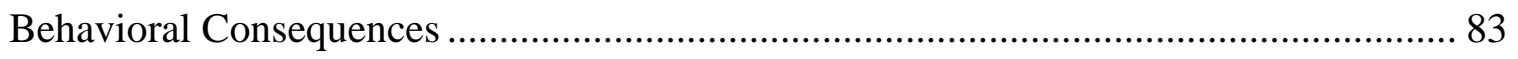

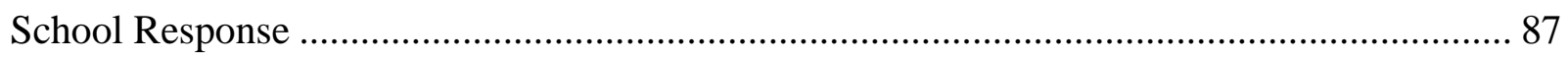

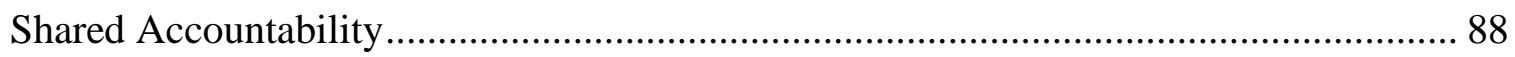

Small Learning Communities and the Freshman Academy ........................................... 91

Supplemental Freshman Transition Supports …………................................................ 93 
Successful Freshmen Transition Programs ........................................................................ 96

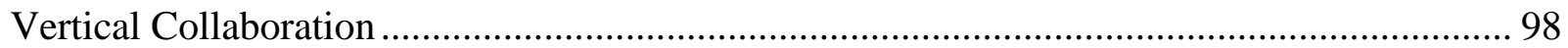

Professional Learning Communities and Teacher Collaboration....................................... 98

Collaboration Focus and Instructional Program Coherence .......................................... 104

Vertical Collaboration and the Transition to High School ............................................. 108

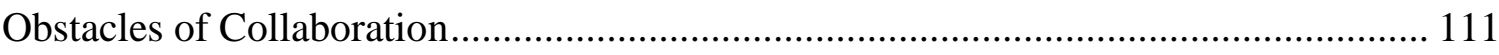

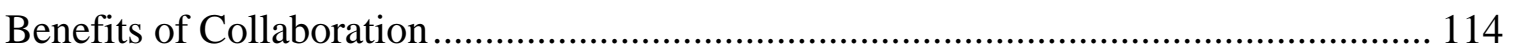

SECTION FOUR: CONTRIBUTION TO PRACTICE …………....................................... 117

Plan for Dissemination of the Practitioner Contribution....................................................... 118

SECTION FIVE: CONTRIBUTION TO SCHOLARSHIP …………………….................... 127

SECTION SIX: SCHOLARLY PRACTITIONER REFLECTION .......................................... 180

Dissertation Influence on Being an Educational Leader ....................................................... 181

Dissertation Influence on Being a Scholar......................................................................... 187

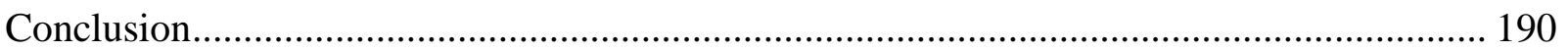

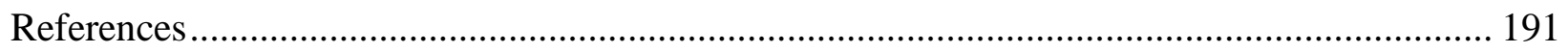

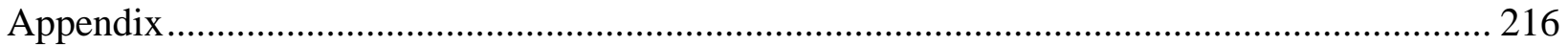

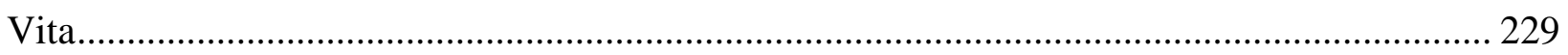




\title{
TEACHER COLLABORATION AND INSTRUCTIONAL PROGRAM COHERENCE ACROSS THE TRANSITION TO HIGH SCHOOL
}

\author{
Kyle Thompson
}

\author{
Dr. Kim Finch, Dissertation Supervisor
}

\begin{abstract}
This study explores to what extent principals perceive instructional program coherence (IPC) and vertical collaboration occur in high schools and their mid-level feeder schools. It also measures IPC and its five components' association with different measures of student achievement. The study took place in the state of Missouri, and 312 principals of high schools with separate feeder middle schools were invited to participate. Electronic and paper surveys as well as public data were collected and analyzed using descriptive statistics, bivariate correlations, and linear regression. The findings show that principals perceive their school's IPC to be fairly strong, but their vertical collaboration needs improvement. The findings also indicate that only particular IPC components such as curriculum, instruction positively influenced graduation rates, academic climate positively influenced the EOC Algebra 1 scores, and vertical collaboration positively associated with higher average freshman GPA's. A major limitation to this study was the low response rate.
\end{abstract}

Key Words: instructional program coherence, vertical collaboration, vertical teaming, collaboration 
SECTION ONE: INTRODUCTION TO DISSERTATION 


\section{Introduction}

The life course consists of transitions that introduce new proximal contexts that can cause a shock to the development of individuals and deflect their long-term life trajectories (Benner, 2011). Educational patterns show the advancement from a mid-level feeder school to high school is arguably the most impactful. High schools start with the freshman "bulge" due to transfer students and retentions, but hemorrhage students to dropping out, largely by the end of the freshman year (Cohen \& Smerdon, 2009; Emmet \& McGee, 2012). Approximately 25\% of students entering the ninth grade, and exceeding 50\% in certain communities, will fail to receive a diploma (Casillas et al., 2012), contributing to the current dropout crisis plaguing society and the economy in the United States. The National Center for Education Statistics (2020) stated that in 2019 "high school dropouts are costly to society, with an estimated lifetime cost close to $\$ 276,000$ per dropout resulting from lower tax contributions, higher reliance on public assistance, and higher incidence of criminal activity" (NCES, 2020, para. 2).

The increase in dropout rates stems from the deficient academic progress many students make by the end of their freshman year (McCallumore \& Sparapani, 2010), especially in urban areas (Neild, Stoner-Eby \& Furstenberg, 2008). Students face credit accumulation requirements for the first time in their educational experience, and course failure results in many freshmen at risk of entering a credit recovery track (Uvaas \& McKevitt, 2013). Neild (2009) stated, "at a minimum, because failed courses must be retaken, the graduation date will be deferred unless the student redoubles his efforts to earn the missing credits in time to graduate" (p. 55). Negative academic outcomes can stem from the consequent struggles roughly $40 \%$ of students' experience due to the transition to high school (Akos \& Galassi, 2004). Research shows, in addition to the higher number of class failures, the change can produce decreased engagement (Benner, 2011), 
negative behaviors (Oakes, 2009; Smith, 2006a), lower attendance (Benner \& Wang, 2014), and declining test scores (Neild, 2009).

A volatile firestorm of interrelated factors contributes to making the entrance to high school so disorienting. Unlike mid-level feeder schools, successful academic achievement is required for grade advancement (Nield, 2009). Coinciding with higher stakes is the increase in both academic rigor (Brown \& Seely, 2010) and autonomy (Chapman \& Saywer, 2001). This combination can cause a damaging disruption to a pupil's learning process, especially with deficient preparation. In addition to the academic change, students face contextual and social disruptions. They must navigate through usually larger unfamiliar buildings and adapt to new procedures and obligations (Gentile-Genitty, 2009). Social convoys change from mid-level feeder schools due to a larger and more diverse population (Langenkamp, 2009). Transitional difficulties may also cause psychological hardships that students must simultaneously navigate (Benner, 2011; Uvaas \& McKevitt, 2013). The life trajectory of each adolescent may depend on their ability to overcome the distress that these and many other factors cause.

Recognizing this challenge, districts have responded using methods ranging from deploying comprehensive school reform models to freshman transition programs (Benner, 2011; Emmett \& McGee, 2012; Kennelly \& Monrad, 2007; McIntosh \& White, 2006; Roybal, Thornton \& Usinger, 2014). Many schools have turned to small learning communities called freshman academies to provide a unified effort of cross-categorical teachers to monitor a smaller proportion of students making the transition into high school (Cauley \& Jovanovich, 2006).

Schools have accommodated these reforms by restructuring the entire high school schedule and staff to produce shared planning time, collaborative intervention, and classes with more familiar classmates (Kennelly \& Monrad, 2007). Some schools have gone as far as restructuring their 
buildings to provide a dedicated geographic area strictly for freshman (Smith, 2007) to simplify their acclimation to the building (McCallumore \& Sparapani, 2010) and alleviate intimidation brought on by intermingling with older students (Kennelly \& Monrad, 2007). Other school responses have included orientations and open houses (Dorman, 2012), student mentoring (Mizelle, 2005), and transitional summer school (Fulk, 2003). Many essential areas need to be addressed for students to successfully transition to high school that a comprehensive program is recommended (Butts \& Cruziero, 2005). Districts are aware of how impactful the transition can be and in response allocate inordinate amounts of time and resources.

Even with extensive dedication to interventions, the academic incoherence between middle and high school teachers could derail a student's educational and life trajectory and could impair the effectiveness of the district's efforts. Teachers are often overlooked in transition program planning (Morgan \& Hertzog, 2001) and the discontinuity in teaching and learning remains prevalent. Educators could compensate with a deliberate effort to build Newmann, Smith, Allensworth and Bryk's (2001a, 2001b) concept of instructional program coherence (IPC), defined as "a set of interrelated programs for students and staff that are guided by a common framework for curriculum, instruction, assessment, and learning climate and are pursued over a sustained period" (p. 299). Within this concept, schools allocate resources to wide-range whole school reform of shared academic vision and consistent means to achieve it (Newmann et al., 2001). In order to increase IPC, cross-grade collaboration or vertical teaming (VT) between teachers is imperative.

Research shows horizontal collaboration, both departmental and interdisciplinary, has a positive effect on student achievement (Ronfeldt, Farmer, McQueen, \& Grissom, 2015;

Moolenaar, Sleegers, \& Daly, 2012). However, vertical collaboration is very challenging due to 
the constraints of time, geography, and resources (Bergman \& Calzada, Lapointe, Lee \& Sullivan, 1998; Berry, Daughtrey \& Wieder, 2009; Vangrieken, Dochy, Raes, \& Kyndt, 2014). Being in separate buildings makes finding innovative and efficient collaborative systems a necessity. Preferably, an IPC framework would already be embedded in the district structure, with a component for vertical collaboration that provides convenient access for teachers to increase the probability of their participation (Berry et al., 2009; Gagda \& Koliba, 2008; Vangrieken et al., 2014). Bottom line, without vertical collaboration, greater IPC becomes more difficult to achieve.

\section{Statement of the Problem}

According to Chapman, Laird, and Kewal-Ramani (2011), "Depending on how and where dropout rates are measured, it is expected that between 9.3 and $43.7 \%$ of freshman will fail to graduate with a regular diploma within 4 years of entering high school” (p. 289). A partial contribution to this outcome is a chain reaction spurred by students' transition from mid-level feeder schools to high school. This transition creates unique but damaging barriers to student success. Comprehensive research on those barriers spans across academic, structural, social, and personal domains (for summary see Benner, 2011). Reactionary research has focused on schools' responses to these findings with various transitional programs (Emmett \& McGee, 2012; Smith, 2006b) primarily at the student and organizational levels. The teacher focus within this literature is mainly limited to suggesting building stronger student-teacher relationships (Wentzel, Tomback, Williams \& McNeish, 2019) and horizontally collaborating with interdisciplinary colleagues to lessen academic and behavioral issues (Emmett \& McGee, 2012).

Converging with the research on the student transition to high school is the concept of IPC in schools. While it is evident that increased cohesion has an overall positive effect on 
student achievement (Madda, Halverson \& Gomez, 2007; Newmann et al., 2001), the pattern in student achievement suggests that IPC suffers the same disruption from the transition from middle to high school that students do. The IPC framework's aim for entire school cohesion (Newmann et al., 2001) aligns with the ideal of many all-inclusive school reform programs that cater to incoming freshman. However, variables exist in the transition to high school that make its impact unique to the rest of the educational progression. The universal nature of IPC framework then becomes a shortcoming because its exclusivity to specific areas of need, like the transition from middle to high school. Newman et al. (2001) recognized that different areas of need require specific attention and may vary accordingly; however, this should adhere coherently with the rest of the institution.

Vertical teacher collaboration to increase IPC across such a unique impactful disruption in a student's educational progression provides the problem of focus of this study. A lack of research exists that demonstrates if districts are using teacher-to-teacher collaboration vertically between separate buildings and if this collaboration impacts student achievement. Also, while studies suggest that mid-level feeder school and high school teachers collaborate (Berry et al., 2009; Dorman, 2012), researchers do not elaborate on the recommended focus of the collaboration, nor what these collaborative practices should entail. In fact, a study by Bergman et al. (1998) showed there was no statistical significance between vertical alignment alone and student achievement. However:

The data suggest that there is a need for qualitative research regarding vertical alignment and collaboration. It is very possible that there was no correlation between alignment and collaboration and scores or growth because the districts that responded to the survey did not share a uniform definition of vertical alignment and collaboration. (p. 28) 
The ambiguity in the definition of vertical alignment and collaboration could be cleared up using the IPC framework. But before specifying what teachers prioritize in collaboration, research needs to be done to determine if it is happening and how it affects students' initial success in high school. Due to separate locations of teachers and the high demand of their time, the efficiency of collaboration is imperative. Knowing how to achieve effective vertical collaboration between interschool teachers would be valuable but is missing in current research.

\section{Purpose of the Study}

The differences between the middle and high school settings along with unforeseen challenges converge to contribute to the distress of students making the transition into high school. Incoming freshmen must reframe their previous understanding and overcome these trials at a time where they are allowed more independence from their parents than ever before (Neild, 2009). The inability to overcome these challenges can lead to consequences that can derail students' life trajectory. These impacts leave school districts scrambling to decide what solutions to implement to ease the transition for students entering high school.

Schools have offered specific transitional programs to aid students in this change. The success of these transitional programs requires collaboration between each building's personnel (Smith, 2006b). Unfortunately, the disjoint structure and relationships across this transition hinder academic coherence between staff. School personnel across the divide need to devote specific attention to create IPC to ease the academic disruption. However, while IPC is

positively associated with student achievement (Newmann et al., 2001), its conceptual framework does not articulate the practical means to produce it. Without effective collaborative strategies in this demanding environment, these teachers will remain incoherent to what 
academic alignment is needed to alleviate the academic disruption for students during their transition to high school.

There were two purposes of this study. The first was to determine to what extent high schools were practicing purposeful vertical teaming with their mid-level feeder schools from the perspective of high school principals. This includes how schools prioritized the four components of IPC within their collaboration at both levels. The second purpose will be to identify if this collaboration had an impact on high school student achievement in the form of freshman course success rate and Missouri's End-of-Course Exam scores in the four core areas. From the previous question, the varying components of school collaboration was measured across student achievement.

\section{Research Questions}

The research questions (RQ) guiding this study were:

1. As perceived by high school principals, to what extent is IPC utilized in Missouri secondary schools?

2. What is the relationship between IPC (as perceived by high school principals) among mid-level feeder school and high school teachers and student achievement as measured by:
a. Graduation rate?
b. Missouri End-of-Course Exams?
c. Freshman grade point average (GPA)?
d. Freshman attendance? 
3. What is the relationship between IPC (as perceived by high school principals) and high school student failure as measured by:
a. Dropout rate?
b. Freshman course failure rate?
c. Freshman out-of-school (OSS) suspension rate?

\section{Conceptual Framework}

While the Life Course Theory is associated in research with the transition from mid-level feeder schools to high school (Benner, 2011), it is from the perspective of the students making the transition. This study examined if and how teachers across the transition academically aligned to ease the disruption for students in such uniquely separated environments. Therefore, IPC was the conceptual framework guiding this study.

\section{Instructional Program Coherence (IPC)}

The concept of IPC was pioneered by Newmann et al. (2001) as, "a set of interrelated programs for students and staff that are guided by a common framework for curriculum, instruction, assessment, and learning climate and that are pursued over a sustained period" (p. 297). The framework consists of four major domains. Curriculum relates to the sequential alignment of academic content that, when properly executed, can improve student achievement (Squires, 2012). Instructional coherence applies to aligning the pedagogical means of achieving a united academic goal (Madda et al., 2007). Common summative and formative assessment allows teachers to analyze results across classrooms and collaboratively learn how to improve their instruction or intervention based on their findings (Jacobson, 2010). Finally, it is well documented that a misalignment of expectations and rigor exists between mid-level feeder school and high school staff (Chapman \& Sawyer, 2001; Cohen \& Smerdon, 2009; 
McCallumore \& Sparapani, 2010; Smith, 2006). Creating a mutual consensus of these learning expectations would be considered a way to improve academic climate.

Three prevailing conditions are evident in IPC schools (Newmann et al., 2001). The first is that leadership and work on the four components progresses toward a common framework. Two goals of collaboration are to increase both democratic unity and teacher efficacy (Stosich, Bocala, \& Forman, 2018). Achieving these would be implementing activities that create a mutual understanding of IPC requirements at both the eighth and ninth grade levels to mitigate the academic disruption students experience from the transition (Mizelle \& Irvin, 2000). The second condition is that ensuring working conditions are conducive toward implementation of the framework (Newmann et al., 2001). Disparate organizational structures provide barriers to collaboration that schools need to bridge through innovative strategic management and common planning time (Smith, Akos, Lim, \& Wiley, 2008). The third condition is to provide resources that are appropriately applied to attain and advance that framework, pertains to the technology tools and training that can be used to improve coherence (Newmann et al., 2001). Research shows that schools are allocating resources toward transitional interventions (DeLamar \& Brown, 2016; Herlihy \& Kennelly, 2007; Kennelly \& Monrad, 2007) and selectively positioning the right teachers within these grade levels (Ellerbrock \& Kiefer, 2014; McIntosh \& White, 2006). It is those teachers' obligation to provide academic alignment to the best of their abilities to help freshmen reach optimal levels academic achievement.

A limitation of IPC was that it is institutionally focused while this study will focus on the transition from mid-level feeder schools to high school. However, Newman et al. (2001), who pioneered the IPC concept cautioned, "The pursuit of greater program coherence must respond to appropriate forms of differentiation and be receptive to new or altered programming for staff and 
for students when clearly necessary" (p. 313). The entrance to high school is a disruption that requires specific interventions to ensure a successful transition for students.

\section{Design of the Study}

To address the research questions, a quantitative study was conducted (Creswell, 2014; Merriam \& Tisdell, 2016). The following section summarizes the study by outlining the setting, participants, data collection methods and tools, and the means of data analysis.

\section{Setting}

The setting of this study took place in public high schools in the state of Missouri. In the 2019-2020 school year, statistics showed that the total number of students enrolled in Missouri schools in the fall was 879,845 . Of those, 612,318 were in kindergarten through eighth grade while the remaining 267,527 were students in grades nine through twelve (DESE, 2020a). The diversity breakdown at the beginning of the 2019-2020 school year for the state of Missouri was as follows: $70.7 \%$ of students were White; $15.7 \%$ were Black; $6.7 \%$ were Hispanic; $4.3 \%$ were Multi-racial; 2.1\% were Asian; and the remaining 0.5\% identified as American Indian or Pacific Islander (DESE, 2020a). Students with limited English Language proficiency made up 3.9\% of the student population. Other student demographics can affect student achievement such as socio-economic status, homelessness, and students that qualify for special education. A little over half of the students in the state of Missouri qualified for free or reduced lunch in the 20192020 school year and $2.4 \%$ of all students were identified as homeless (DESE, 2020a). Finally, $13.5 \%$ of students were classified as special education students (DESE, 2020a).

The Missouri Department of Elementary and Secondary Education (DESE) requires high school students to take two types of standardized assessments based on age: End-of Course Exams (EOC) and the ACT (DESE, 2020b). In the 2018-2019 school year, the percentage of 
students who scored proficient or advanced in English Language Arts II was 59\%; in Algebra I it was 45.3\%; Algebra II had 50\%; and Biology had 39.2\% (DESE, 2020a). The most recent data showed that in 2018, $65.5 \%$ of students scored proficient or advanced in Government, as the data were not available for the 2018-2019 school year (DESE, 2020a). Missouri uses the ACT as a comparative test nationally. In the 2018-2019 school year, Missouri was the 27th ranked state with an average composite ACT score of 20.8, which was slightly above the national average of 20.7 (Statista, n.d.). Missouri's 4-year graduation rate in 2019 was 89.58\% (DESE, 2020a), meaning an estimated 27,783 high school students in the state of Missouri would not graduate within four years. In response, many Missouri schools are adopting freshman transition programs (Biermann, 2010; Springfield Public Schools, n.d.) to reduce the amount of credit recovery taken later in high school.

Enrollment plays a significant role in determining the structure, hiring, and funding for school districts. The standard maximum class size for the state is 33 students per class in grades 7-12. However, the desired target is 25 students per class (DESE, 2020b). This standard, along with the total school enrollment, contributes to the budgeted number of full-time teachers in each district. In the fall of 2019, there were 69,849 classroom teachers broken down by school level in the following manner: 20,377 high school teachers, 1,957 junior high school teachers, 10,364 middle teachers, 33,139 elementary teachers, 1,547 early childhood teachers, and the remaining 2,466 teachers were classified as "other" (DESE, 2020a). The overall K-12 student-to-staff ratio in the 2019-2020 school year was approximately 17:1 (DESE, 2020a). The average experience of teachers was approximately 12.9 years, which is a decrease due to the decline of teacher retention rates of those with at least five years of teaching experience (DESE, 2020a). 
The educational pathway of students consists of a continuous progression that builds upon itself yet is disrupted by the transition to separate schools as students are promoted. In the state of Missouri, school districts follow different structural patterns based on geography, enrollment, and resources. Currently, Missouri houses 518 school districts, and high schools are classified by size (DESE, 2020a). Larger school districts tend to have one or more separate feeder middle or junior high schools. In certain instances, smaller school districts consist of one location in which all students from pre-kindergarten through the 12th grade are housed. In the 2019-2020 school year, Missouri schools consisted of 568 high schools, 49 junior high schools, 295 middle schools, and 1,221 elementary schools (DESE, 2020a). Like other demographics, these different district structures, and students' transitions between them, have unique impacts on educational experience and achievement (Alspaugh, 1998).

\section{Participants}

The participants in this study were all the principals in Missouri public high schools whose structure houses grades 9 through 12 within their building and have separate geographically located mid-level feeder schools. These principals must have held an administrative position within their current high school for more than one year. Currently there are 563 high school principals in the state of Missouri. To find the specific principals that fit the required criteria, the state school directory from DESE was utilized. A filtration system determined which high schools consisted of the appropriate grades. From that list, the researcher went through and removed all non-traditional high schools, such as technical schools and career academies. Finally, the remaining schools were analyzed to see if they had mid-level feeder schools in separate geographic locations. Overall, 312 high schools qualified to create the population for this study and the principals of those schools were invited to participate. 
An requirement added to the study that mandated participating principals to have over one year of administrative experience in their building. This was done for two reasons. The first is that new principals may not yet have an accurate portrayal of the IPC of their school. The second was that due to the Covid-19 pandemic in 2020, this study required data from the 20182019 school year. To omit first year principals, section one in the survey (see Appendix) consisted of a qualifying question to find the principal's years of experience. If it was a year or less, they were redirected to the end of the survey and were void from participant pool. As recommended by Fink (2017), technology was applied to cover the large geographic area and large population of this study. Email addresses were obtained from the DESE directory and a precursory email was sent to all principals inviting them to participate in the study.

\section{Data Collection}

Electronic Survey: A survey was electronically sent to all high school principals using Qualtrics. A valid reason to conduct surveys is to collect data that helps plan and guide programs (Fink, 2017). One intention of this study was to provide data that help school districts implement and improve interventions that address the disruption caused by the transition to high school. The survey consisted 35 questions (27 ordinal, 7 numerical, and 1 short answer) broken down into three sections (see Appendix).

- Section 1: Qualifying Questions. This section consisted of an informed consent question and a question to eliminate participants with one year of experience or less within that school. Those principals were redirected to exit the survey.

- Section 2: School Data. This section gathered all freshmen data not obtainable by the researcher through DESE. It also included a request for contact information for 
someone within the district with this data in case it was not easily accessible by the principal.

- Section 3: Instructional Program Coherence. This section measured the IPC focus of high school collaboration. It was adapted from Newmann et al.’s (2001) Likert-scale survey by incorporating different IPC components: curriculum, instruction, assessment, academic climate, and vertical collaboration. Subscales of the five IPC components were created using the following questions:

○ Instruction: Q9, Q16, Q23, Q26, Q30

○ Curriculum: Q10, Q12, Q15, Q21

○ Assessment: Q11, Q13, Q17, Q22

○ Academic Climate: Q14, Q18, Q19, Q20, Q24, Q25

○ Vertical Collaboration: Q27, Q38, Q29, Q31, Q32, Q33, Q34, Q35.

The survey was conducted from December 1, 2020, to February 28, 2021. Respondents received email reminders to complete the survey through Qualtrics on average of every two weeks. The duration was extended in attempt to gain the amount of responses that validly represented the population (Field, 2018; Fink, 2017). With approximately 3 weeks remaining in the survey duration, phone message reminders were left to increase participation. Fifteen messages were left in the span of two evenings. After three days, zero of those participants responded so phone calls were ceased.

Paper Survey: At the halfway point, paper surveys identical to the electronic survey, were mailed to the 295 principals who had not yet responded to increase the response rate (see Appendix). They were four pages in length, and a self-addressed stamped envelope was included 
for their return. Ten surveys were returned of which six were usable in this study due to full completion and the participants and meeting the required criteria.

DESE Records: To decrease the length of the survey, dependent data was obtained by the researcher through online DESE records (DESE, 2020a). These records included graduation rates, dropout rates, freshman course failure rates, out-of-school suspension rates, attendance rates, and EOC scores. The researcher also used the free-and-reduced lunch rates and enrollment data from DESE in this study.

Ethical consideration. Principals agreed to an informed consent form before any form of participation. The researcher followed Seidman's (2013) Eight Major Parts of Informed Consent as well as the University of Missouri Institutional Review Board standards. Participants had full transparency and withdrawal rights throughout. The questionnaire from Fink (2017, p. 24) provided ethical technological guidelines for this study. By using school emails provided by DESE, the researcher was assured of their authentication. Qualtrics was used through the University of Missouri to ensure secure data storage and encryption.

\section{Data Analysis}

Statistical analyses were conducted for all three research questions using Statistical Package for the Social Sciences (SPSS). The objective of data and collection was to answer the research questions, and therefore, each research question and the corresponding analysis are shown in Table 1. Socioeconomic status was controlled throughout the analysis using the free or reduced lunch data from DESE. 
Table 1

Quantitative Analysis of Data to Address Research Questions

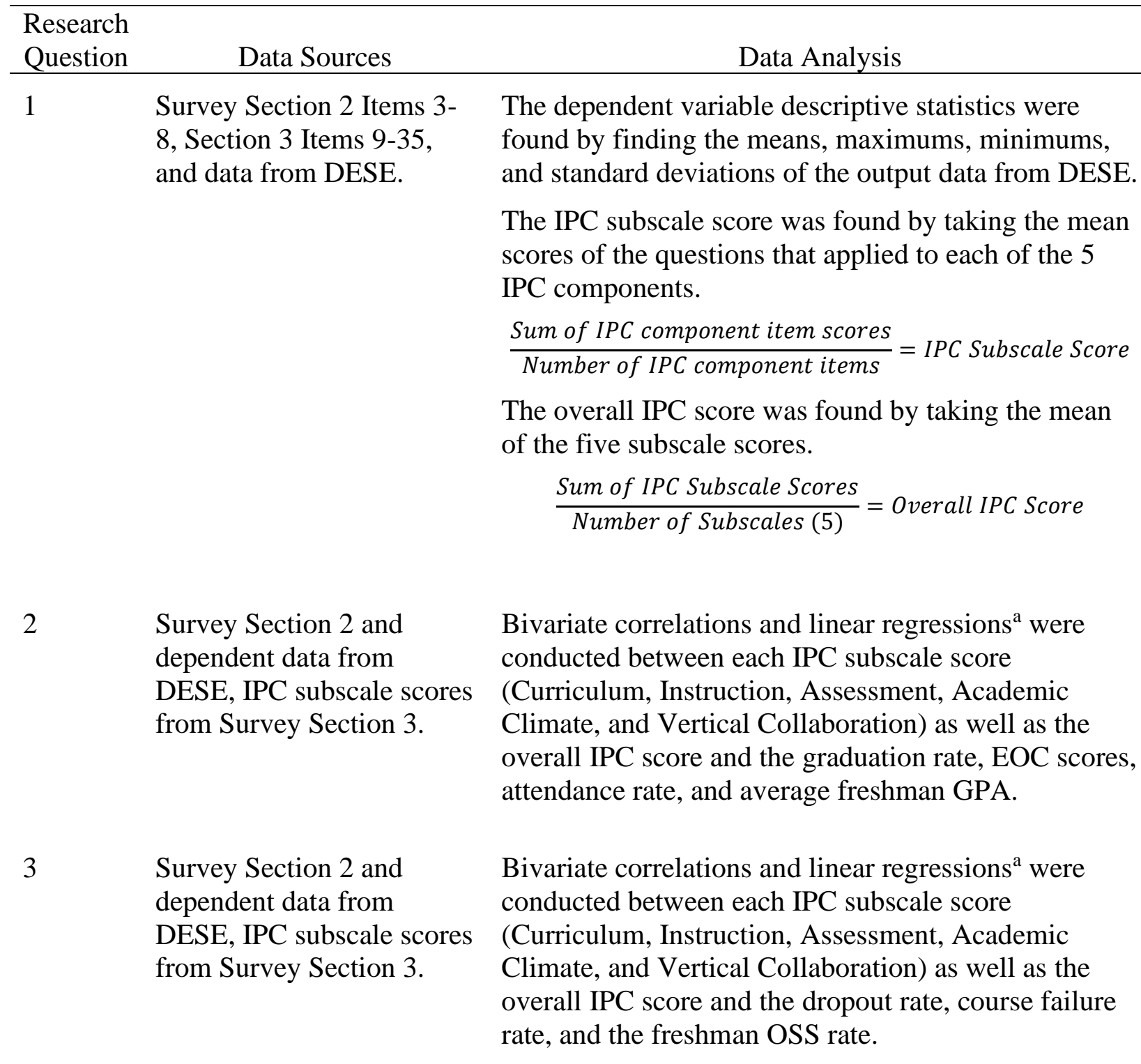

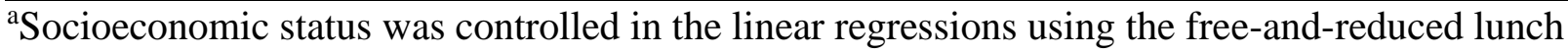
data for each school obtained from DESE (DESE, 2020a). 


\section{Limitations}

The most glaring limitation to this study is the low response rate. This can be attributed to many factors. In the spring of 2020, the Covid-19 pandemic hit the United States which forced schools to face unforeseen challenges. This carried over into the 2020-2021 school year where high school principals' focus was adapting their school structures and protocols to conduct school in the safest manner possible. The researcher received numerous emails where the sender apologized for their inability to participate in the study because of the unprecedented situation they were facing at their school due to the pandemic. Difficulties were compounded as the survey coincided with the transition from the fall to spring semester and winter break. Many schools in the state of Missouri were adjusting their operations based on data and observations from the first semester and health guidelines, which provided further attention from principals.

Also contributing to the low response rate was the low prioritization by principals of this topic as being one in need of focus. The lack of necessity can result from many factors. Schools operate under a district structure that has a curriculum position that is often attributed with the responsibility of ensuring learning continuity between building levels. Districts operate with an understanding that the state curriculum is aligned accordingly so that all content is covered. In addition, many high schools are at different points of implementing programs to ease the transition for incoming freshmen and may have seen some positive results or the results are pending. These programs often overlook the role IPC can play in the disruption and therefore do not incorporate the necessary vertical collaboration. Finally, very little research exists examining the specific dynamics of the relationship between mid-level feeder school teachers and high school teachers when pertaining to academics. With this being uncharted in research, it is 
unknown to principals and therefore not seen as a priority. Further research could expose a need for their future participation.

To try to increase the response rate, the researcher offered help in finding the data through contacts in the district, sent out paper surveys, made phone calls, and streamlined the survey to omit information that could be found through DESE. Accessibility to complete the survey was also expanded from the initially planned three weeks to an overall duration of three months with numerous attempts to garner increased participation. However, even with these modifications, the response rate remained below the recommended target and forced a change in the data analyzation.

Another limitation to this study is that it is a singular cross-sectional study as opposed to a longitudinal one. Due to the Covid-19 pandemic, the 2019-2020 End-of-Course (EOC) exams were cancelled by DESE and many school districts altered their grading scale to compensate. To respond, this study had participants pull student data from the 2018-2019 school year and required only principals who had been in their school's administrative cabinet for more than a year. Another reason is that collaborative programs tend to have better student achievement results the longer they are sustained (Jacobson, 2010). In a longitudinal study, the trends of student achievement growth as programs progress could be examined. Schools that recently implemented freshman transition interventions may not have seen the results yet, and that could alter the outcome of the analysis of the collaborative efforts relationship to student achievement.

The participants' subjective interpretation of the definition of intentional collaboration is another limitation. While it was defined by the researcher, principals may define it differently based on the context of their setting and staff. Also, collaboration may occur outside of professional development or common planning time that will not counted by the principal in this 
study yet may influence student success. In the reverse effect, principals may embellish the amount of collaboration or IPC level to obtain a more socially desirable impression of their school and their work. A further limitation on this study was the researcher's assumption that school districts are overwhelmingly not addressing the academic continuity between mid-level feeder school and high school teachers. This created a researcher bias, which was addressed throughout every portion of the study.

\section{Key Terms}

In studying collaboration between teachers across the mid-level feeder school and high school transition, many common terms appear throughout research. For clarity, the following is a list of key terminology guiding this study:

- Mid-level Feeder Schools consist of schools of any structure that feeds into a high school that starts at grade 9. Feeder school types, such as middle schools or junior high schools, can differ drastically in structure and operations. Junior high schools usually start at 7 th grade while middle schools start earlier at 5 th or 6th grade. Middle schools also tend to operate with teacher teams in more communal settings whereas junior high schools tend to follow a more bureaucratic system similar to high school. Mid-level feeder schools include all middle level schools, regardless of type, that end at grade 8 and are separated geographically from their receiving high school.

- Instructional Program Coherence (IPC) is when "a common instructional framework guides curriculum, teaching, assessment, and learning climate. This framework combines specific expectations for student learning with specific strategies and materials to guide teaching and assessment" (p. 299) and are pursued over a sustained period of time (Newmann et al., 2001). 
- Collaboration - "professional interaction with colleagues that focuses on refining and improving classroom instruction, curriculum, and supports for students" (Johnson \& Tsai, 2018, p.3)

- Vertical Collaboration - Planning, aligning, and implementing ideas and strategies pertaining to curriculum, instruction, assessment, academic climate, and student information sequentially from one grade level to the next from grades prekindergarten through grade 12 (Bergman et al., 1998; Berry et al., 2009).

- Vertical Teaming - people from different levels within an organization who are committed to a common purpose, performance goals, and approach for which they hold themselves mutually accountable to achieve the desired objective of improving student learning by developing the instructional skills and abilities of educators (Crawford, 2011).

\section{Significance of the Study}

A plethora of research suggests collaboration between mid-level feeder school and high school teachers to ease the disruptive assimilation process incoming freshmen face because of the transition (Brown \& Seely, 2010; Chapman \& Sawyer, 2001; McIntosh \& White, 2006, Oakes, 2009; Smith, 2006a). However, a gap exists if teacher collaboration between feeder and high school teachers is taking place, what the specific focuses of this collaboration are, and if different collaborative elements influence different measurements of student success. The IPC provides a conceptual model, but this study provided insight crucial in creating an adjustable guide that clarifies the realistic academic elements teachers and school administrators can adapt to stabilize the learning process for students entering high school. The demanding environment is requiring teachers to be more resourceful with their time and efforts. Teachers bordering the 
transition to high school must navigate the unique barriers and ambiguity it creates. This study could provide innovative, efficient, and effective vertical collaboration practices that schools can try to fit this setting. Future studies can measure which IPC and collaborative components can be measured in relation to other social, psychological, or structural factors found in research. For example, Anderson et al. (2019) showed that middle school students whose self-efficacy declines as they enter high school affects their academic outcomes. Further studies could illustrate how strategies found here could associate to building student success that improves that self-efficacy.

An evaluative measurement tool could be derived from this study to assess if departments within this setting are doing their part to build IPC, since it has been proven to improve student achievement (Newman et al., 2001). At a higher level, an evaluative tool may offer insight to policy makers to implement guidelines that build IPC between mid-level feeder schools and high schools. The subsequent discovery of the strategies that work could be institutionalized across the district. This also will prioritize how to allocate resources more effectively according to what IPC components produced higher student achievement results for incoming freshman. The researcher's current institution could increase continuity and awareness between middle schools and high schools by better informing the teachers of student performance ahead of time. This will allow for additional preventative measures by high school teachers to keep students on track with proper student placement in high school courses and more precise remedial instruction based on previous data. Proper IPC enables mid-level feeder school teachers to adjust the rigor and preparation techniques before their students enter the world of credit accumulation. With the framework provided, attention could be devoted to creating efficient embedded practices within the current constraints of our educational structure. 


\section{Summary}

An adjustment period occurs for every student entering high school. The difficulty of this transition associates with students' likelihood to drop out of school (Alspaugh, 1998). With these implications, schools have made concerted efforts to ease this transition for students. However, the efforts will be futile if the learning process between mid-level feeder schools and high schools remain too disjointed for students to overcome. Building IPC has been known to increase student achievement (Newmann et al., 2001). There remains ambiguity on what the most effective IPC components these cross-transitional teachers should focus on and the practical strategies necessary to ascertain improved IPC across the building boundaries. Guided by the IPC Framework, this mixed methods study quantitatively measured if vertical collaboration was occurring within the high school, between high schools and their mid-level feeder schools, and to what extent. The researcher identified if there was a relationship between the different IPC elements and student achievement as measured by a variety of barometers. Overall, this study provided clarity on the teachers' collaborative focus in alleviating the academic disparity between mid-level feeder schools and high schools to help ease the transition for students. 
SECTION TWO: PRACTITIONER SETTING 


\section{Introduction}

The setting of this study influences school districts' response to combat the disruptiveness created by the transition from mid-level feeder schools to high school. Schools and leaders function within a setting where many of their decisions are at the mercy of the system in which they are bound. In order to make progress in a virtually unchangeable environment, Missouri educational researchers and practitioners alike need to understand the setting in which they operate. This section examines that setting by first giving a brief history of high schools in the United States and outlining the current educational system in the state of Missouri. That will be followed by an organizational analysis framed through the structural lens (Bolman \& Deal, 2013) and a leadership analysis using the Adaptive Leadership Model (Heifetz \& Laurie, 2010; Northouse, 2016). It will conclude with research and practical implications for educators in the state of Missouri.

\section{History and Background of the Organization}

The system under which school districts operate contributes to the educational pathway students follow. In this section, the history of high schools in the U.S. and the current make-up of the state of Missouri Education System will be examined.

\section{History of U.S. High Schools}

The first public high school in the U.S. was enacted in Boston in 1821 (Goldin \& Katz, 2008b; Office of Vocational and Adult Education, OVAE, 2003). The early 20th Century a dramatic expansion of the number of high schools in the United States became what is now known as the "High School Movement" (Cohen \& Neufield, 1981; Goldin, 1998; Goldin \& Katz, 2008; 2011; OVAE, 2003). Led by John Dewey in the Progressive Era, secondary school enrollment grew from $18 \%$ in 1910 to $71 \%$ in 1940 (Goldin, 1981; Goldin \& Katz, 2011). 
Graduation rates in the United States grew from 9\% of Americans in 1910 to $40 \%$ in 1935 (Thattai, 2017) and another $11 \%$ in the following 5 years (Goldin, 1981; Goldin \& Katz, 2011). In fact, the approximate number of students enrolled in American high schools in 1910 doubled by 1920 (Thattai, 2017). High schools were being so rapidly built in cities around the country, that rural parents would move closer to a city so their child could attend (Thattai, 2017). In 1932, the federal government found there were 132,000 school districts in the nation in their first count and approximately $20 \%$ of them contained a high school (Goldin \& Katz; 2011). No other country had enrollment growth remotely close to that of the U.S. due to its installation of high schools (Goldin, 1981; Goldin \& Katz, 2011).

Before 1910, the common reason to attend high schools was they offered classical curriculum that was required by many colleges (Goldin, 1981). However, a shift occurred that changed the narrative of public high schools to one that became a terminal degree, preparing students for life in the workforce as well as college (Goldin, 1981; Goldin \& Katz, 2008). The new philosophy adopted by many states was high schools were preparation for adulthood, no matter where that path may lead upon graduation (Cohen \& Neufield, 1981, OVAE, 2003). Concurrently, child labor and truancy laws were being enacted that brought even more enrollment to public high schools (Goldin \& Katz, 2011; OVAE, 2003). Eventually the enrollment increased so dramatically, it created problems such as over-populated buildings, overwhelming transport costs, and a more diverse general curriculum (Goldin, 1981). Ironically, the solutions to remedy the problems created by the rapid "High School Movement" growth was to increase the "High School Movement" by investing more resources and making it more accessible. 
The push for public secondary education was also a grassroots effort, with only $16 \%$ of funding being produced federally in 1925 (Thattai, 2017; Goldin, 1981). The freedom from federal control allowed state and local agencies to provide post-elementary schooling that was flexible, general, and widely applicable skills that were not occupation specific, but could suit the needs of their geographical context (Thattai, 2017). However, the need for high school education to be made accessible to all was seen at the state level. State officials also recognized the need for the preparation of students to result in transferrable knowledge and skill. In response, states started coordinating secondary education at the district and state levels by implementing standards for what constituted a high school, and the requirements for promotion and graduation (Goldin \& Katz, 2011). It was during this period when the core structures and regulations of the modern education were created (Goldin \& Katz, 2008; OVAE, 2003). In fact Goldin (1998) stated, "the modern form evolved in the early 1900s and diffused so rapidly that the high school of the 1920s bears a far greater resemblance to that of the late twentieth century than it does to that of 1900" (p. 350).

While parallels can be drawn between contemporary secondary schools and those during the "High School Movement," many events led to changes in curriculum, structure, and enrollment that results in our high schools today. In 1892, the National Council of Education created a board of Ivy League professors known as the Committee of Ten constructed a collegetrajectory model of standards to be used in secondary schools (OVAE, 2003). These standards were modified with the paradigm shift caused by the "High School Movement." The National Education Association appointed a group called the Commission of Reorganization of Secondary Education, who initiated The Cardinal Principles of Secondary Education in 1918 (OVAE, 203). These principles emphasized the high school's role in custodial care of the students and the "life 
adjustment" education that carried American high schools through World War II; this role remains the foundation for many functions of education today.

The post-World War II era saw many changes in education. Charles Prosser, the first director of the Federal Board of Education, famously supported life-adjustment education in 1945 at a conference on the U.S. Office of Education in 1945 (OVAE, 2003). This embedded American high schools in the mind of the public as democratic and universally accessible agencies that produced standardized outputs with little outside effort (OVAE, 2003). This era also saw the attendance rates grow to over $75 \%$ of eligible students (Cohen \& Neufield, 1981). Similar to the 1920 's, the rate of college entrance was smaller; however, the college enrollment was actually larger due to the number of students who were now able to attend high school but did not pursue higher education (Goldin, 1981). Many factors contributed to this, such as newly qualified high school attendees being deemed not "college material." However, Cohen and Neufield (1981) contested it could be due to the emphasis on life-adjustment education making social promotion the means to advance students who were deficient academically. They stated that in the socially sheltered environment high schools morphed during this era; if students dropped out of high school, it was due to their nonattendance rather than their performance not warranting promotion (Cohen \& Neufield, 1981).

The decreasing level of performance expected from high school students that Cohen and Neufield referred to became evident in the 1950's and 1960's when comparing progress to other countries. Since the turn of the century, the "High School Movement" made the U.S. the leader in education. Due to the universal post-war reform implementing rigorous academic standards, U.S. students were now being challenged academically from countries in Europe and Asia (OVAE, 2003). This was symbolized with the Soviet Union's launch of Sputnik in 1957, which 
forced the U.S. to examine their education system (Cohen \& Neufield, 1981; OVAE, 2003). While graduation rates were increasing, math and science progress as measured by the National Assessment of Educational Progress, was decreasing (OVAE, 2003). In response, the U.S. Federal Government enacted the Elementary and Secondary Education Act of 1965, which pumped federal money into local school districts (Thattai, 2017). Along with it came more course requirements, stricter testing methods, better facilities, and educational tracks that separated gifted students from those they classified as not college bound (OVAE, 2003). Many of these requirements are still being utilized in today's high schools.

From the 1950's to today, major events and legislation have been passed that have also contributed to the contemporary high school landscape. The Civil Rights Movement brought on the Supreme Court case of Brown v. Board of Education (1954) that exposed the unconstitutional inequities of segregation in schools and practically abolished segregated schools in the U.S. by the 1970's (Thattai, 2017; Cohen \& Neufield, 1981; OVAE, 2003). While segregation in schools does not officially still occur, racial disparities still do. Currently, closing the achievement gap is a priority metric to which a school district's success is measured. Another metric used is to close the achievement gap for children with learning disabilities due to the Education of All Handicapped Children Act in 1975 (Thattai, 2017; OVAE, 2003). This law mandated full educational opportunities for all students with disabilities and was amended in 1986 to extend to younger children (Thattai, 2017). In 1990, The Individuals with Disabilities Education Act (IDEA) changed the label "handicap" to "disabilities" (Thattai, 2017).

The 1970's saw a push in private, often religious, schools from those who believed public high schools no longer held the character to educate (Cohen \& Neufield, 1981). In 1983, the government released a report called a Nation at Risk that was reminiscent of the Committee of 
Ten's ideals in the 1890's and prompted imposing higher academic standards for all students (OVAE, 1983). These standards emphasized cultural literacy that has remained in classroom practice, curriculum, and textbooks through the 21st Century (Thattai, 2017). However, progress has been slow and uneven since the report 1983. In 2001, the No Child Left Behind Act (NLCB) was implemented with the goal that $100 \%$ of students would be measured as proficient by the year 2014 (Thattai, 2017; OVAE, 2003). NLCB was eventually replaced because achieving $100 \%$ proficiency in universally imposed standards has proven to be unrealistic in education, especially when intervention for negative results included punishing school districts (Thattai, 2017). Overall, the virtues that historically have led to the expansion and enhancement of high schools in the U.S. still contribute to their success, but also their failure.

\section{The State of Missouri Educational Structure}

School districts in Missouri operate under a set of rules governed by the Missouri State Board of Education. This eight-person, governor appointed board is responsible for defining academic performance standards and assessment required for schools and setting policies for the Department of Elementary and Secondary Education (DESE). The Missouri DESE is the administrative arm of the Missourit State Board of Education and is the conduit of educational services between educators, government agencies and officials, and community leaders and citizens (DESE, 2020b). The Missouri State Board of Education also appoints the Commissioner of Education to lead DESE and oversee the management of its two divisions, Financial and Administrative Services and Learning Services (DESE, 2020b). Within these two divisions lie 11 subdivisions that have specific responsibilities (see Figure 2.1). The Office of College and Career Readiness provides assistance on implementation of the state mandated curriculum and assessment program which outlines the progression students follow for promotion, while the 


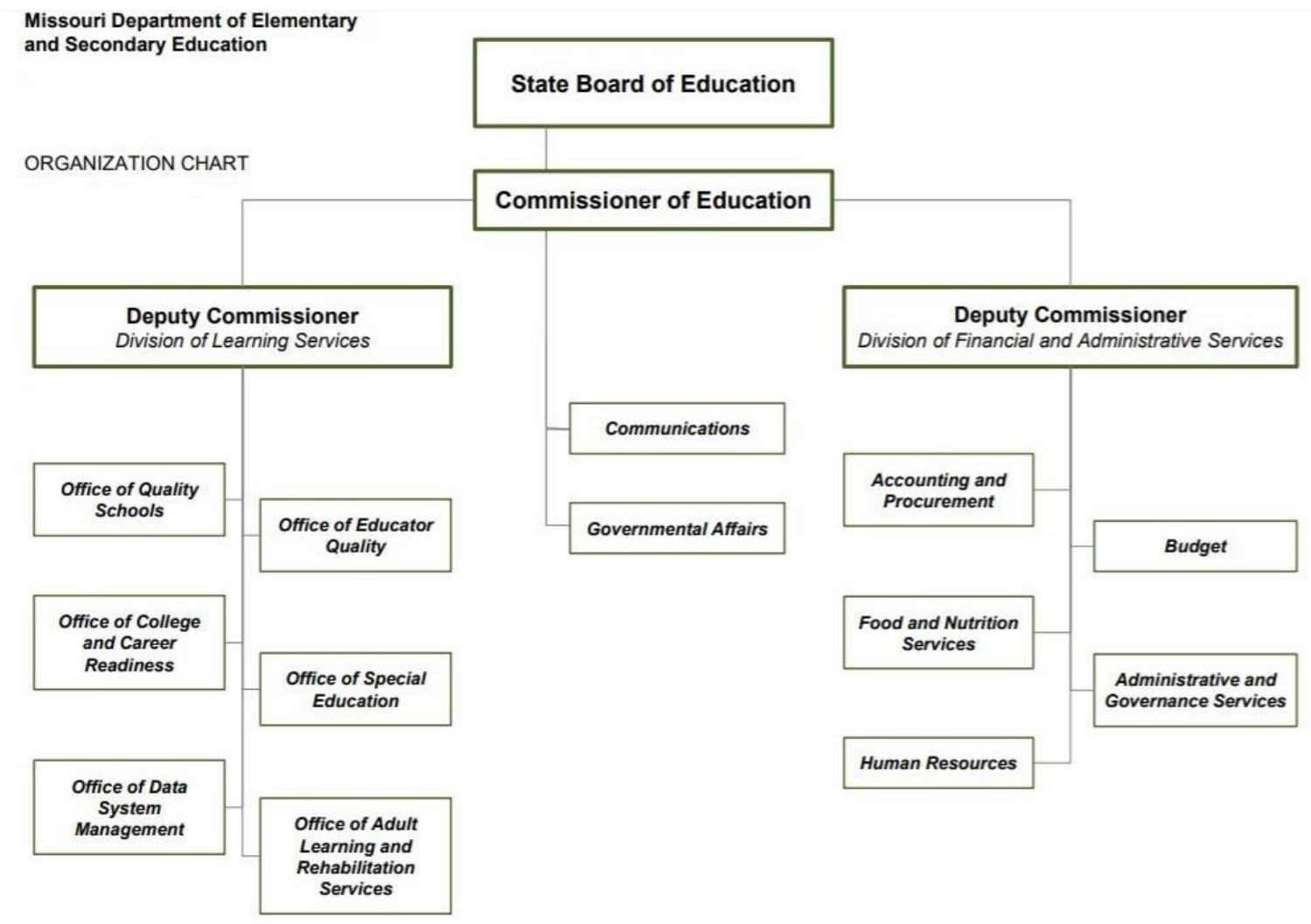

Figure 2.1. The two divisions and eleven subdivisions of the Missouri Department of Elementary and Secondary Education organizational structure. Reprinted from: "Department Offices," by Missouri Department of Elementary and Secondary Education, 2020 (https://dese.mo.gov/sites/default/files/DESE_orgchart.pdf).

Office of Quality schools manages the state's accreditation system and administers supplemental state and federally funded programs (DESE, 2020b).

The Missouri State Board of Education and DESE set certain required criteria for all schools in the state to follow. Since 2010, in order to graduate high school, all students are required to accumulate a minimum of 24 credits by successfully completing courses. The four traditionally core disciplines have their own requirements. Students need to accrue 4 English and language arts credits, 3 mathematics credits, 3 social studies credits, and 3 science credits (DESE, 2020b). The remaining credits consist of non-core required courses and electives. 
Students do not have a semester requirement when it comes to completing coursework as some can finish early or some can take more time. However, they are required by law to attend school until they are 17 years of age or have completed 16 credits toward graduation. The maximum age in which free education must be offered is 21 (NCES, 2020). While the state requirements are the minimum, districts can implement additional requirements or follow more rigorous guidelines based on higher education requirements.

The promotion requirements in high school are vastly different from those in mid-level feeder schools, which raises the rigor and pressure on students making the transition. Students enter a credit accumulation world for the first time (Neild et al., 2008; Emmett \& McGee, 2012) and Missouri is no different. Since 2010, The Missouri Consultants for Education (2020) helped implement a policy for mid-level feeder school retention that states:

1. A list of those students who are not meeting grade level objectives in reading, language arts or mathematics will be given to the building principal by the October, January and March reporting periods. With respect to any student who is reported as not meeting grade level objectives in reading, language arts or mathematics:

a. The teacher, principal and counselor will meet to review the student's academic record, current test scores and work samples.

b. The parent/guardian will be notified as soon after the review as possible that retention is being considered. A meeting with the parent/guardian will be scheduled. The parent/guardian will be informed that the student is not meeting grade level objectives and will be retained in the same grade unless there is strong and positive improvement in the student's work. 
c. A follow-up conference for the parent/guardian will be scheduled with the principal or the principal's designee to review the student's progress.

d. An academic program including remediation will be offered to the student.

2. In recommending promotion or retention, these factors will be considered:

a. Academic achievement in all subject areas, especially attainment of grade level objectives, as determined by tests, teacher assignment, and work samples.

b. Chronological age.

c. Study Habits.

d. Attendance.

e. Social and emotional maturity.

f. State-mandated retention requirements for primary/mid-level feeder school students.

3. The decision for retention will be made by the principal and the classroom teacher in accordance with the above-referenced factors, and written notification of retention will be sent to the parent/guardian. (para. 1)

Under this system, students can only be retained through these sets of criteria and can be promoted regardless of class performance until they reach high school.

In addition to setting the requirements for graduation, the Missouri State Board of Education also provides school districts with a curriculum to follow. In 1996, the state adopted the Show Me Standards (DESE, 2020b). The Missouri DESE (2020b) states, "The standards are built around the belief that success of Missouri's students depends on both a solid foundation of knowledge and skills, and the ability of students to apply their knowledge and skills to the kinds 
of problems and decisions they will likely encounter after they graduate" (para. 1). These standards are considered the blueprint for which curriculum should be built in order to achieve the following four goals of student success:

- Students in Missouri public schools will acquire the knowledge and skills to gather, analyze and apply information and ideas.

- Students in Missouri public schools will acquire the knowledge and skills to communicate effectively within and beyond the classroom.

- Students in Missouri public schools will acquire the knowledge and skills to recognize and solve problems.

- Students in Missouri public schools will acquire the knowledge and skills to make decisions and act as responsible members of society. (DESE, 2020, para 1.)

The latest iteration of curriculum built from these standards that should provide schools with a road map of learning expectations in each course is called the Missouri Learning Standards (DESE, 2020b). Currently, grade-level standards are provided for English language arts, mathematics, science, social studies, personal finance, computer science, fine arts, physical education and health, and world languages (DESE, 2020b).

In 1993, Missouri created a statewide assessment program designed by grade level and discipline that measured student and district performance across state learning standards (DESE, 2020b). This is known as the Missouri Assessment Program (MAP). Originally, the MAP tests were coordinated to only assess certain subjects at different grade levels from 3rd grade through 11th grade. In accordance with the enactment of the No Child Left Behind (NCLB) legislation in 2001, reading and mathematics tests were to be given in every grade from 3rd through 8 th grade and at least once in high school. Science was to be assessed at least once during 3rd 
through 5th grade, at least once during 6th through 9th grade, and at least once during 10th through 12th grade (DESE, 2020b). In the 2008-2009 school year, science tests were increased and administered to every grade level through the 8th grade. At the high school level beginning in the 2008-2009 school year, the MAP tests were replaced with the End-of-Course (EOC) assessments. Since 2014-2015, school districts were required to have every student complete the EOC assessments in Algebra I (or Algebra II if Algebra I was completed prior to high school), English II, biology, and government in order to graduate (DESE, 2020b). However, the state also offers EOC assessments in English I, geometry, American History, and physical science.

The local level of education includes the district and schools who are overseen by a governing board of education. This board consists of a varying number of elected members based on the size of the district they are representing. Beckham, Willis, and Weeks (2007) summarized the responsibilities of local school boards in relation to the state structure:

Sources of authority that influence the duties and responsibilities of the local school board include state and federal constitutions, legislative enactments, rules and regulations promulgated by the U.S. Department of Education and the state board of education, and legal interpretations by judges, attorneys general, and administrative agencies. A school board functions locally, within the confines of the state's delegation of power and the geographical boundaries of the district but is a legal agency of the state and thus derives its power from the state's constitution, laws, and judicial decisions. By state legislative enactment, school boards are delegated power and authority to develop policies, rules, and regulations to control the operation of the schools, including system organization, school site location, school finance, equipment purchase, staffing, attendance, curriculum, extracurricular activities, and other functions essential to the day-to-day 
operation of schools within the district's boundaries. Boards may also be authorized by the state legislature to levy taxes, invest resources, initiate eminent domain proceedings, acquire land, and assume bonded indebtedness. (para. 5)

While school districts act in accordance with state and federal structures and regulations, contextual factors such as enrollment, geography, community, and budgets make each district unique, and they should therefore operate differently to best suit student needs. The state binds local districts only to a core set of regulations, so schools should be encouraged to develop their own distinctive character and pursuit of shared goals (Hadderman, 1988).

\section{Organizational Analysis}

The Missouri high school education system is a hierarchy of smaller systems bound in an extremely large system. It is composed of extremely diverse school districts with their own set of unique students, staff, and communities. With these differences comes difficulties that are particular to each district that can also deter progress to the overarching goals of Missouri education. All levels of the Missouri education system converge to support and hinder the easing of transitional academic disruptions in a school district. The transition from mid-level feeder schools to high school has so many uniquely imposed external influences that school districts need to negotiate to ensure student success. This analysis will be conducted through Bolman and Deal's (2013) Structural Frame, supplemented by their Political Frame.

\section{Structural Frame}

The overarching goal of school districts is to promote students through graduation and prepare them college and career, so they are ready to make and act on informed decisions toward a successful future. Every decision and action of everyone involved should be aimed at achieving these goals for every student. This follows Bolman and Deal's (2013) structural 
assumption that "organizations exist to achieve goals and objectives" (p. 45). Education faces challenges such as preparing students for an ever-changing environment with limited resources. The complex configuration and competitive educational landscape make achieving the goal of student success a wicked problem for educators and administrators alike. Head and Alford (2015) defined a Type 2 wicked problem, "where the definition of the problem is clear, but the solution is not-typically because the relevant cause and-effect relationships are hard to discern - and therefore learning and discussion are required by both the governmental managers and the stakeholders they lead" (p. 717). The problem is clear, to get every student to succeed and graduate, yet seemingly unattainable. Everyone in the Missouri education system is theoretically unified under this goal, but the means in achieving it are incredibly difficult to find for many reasons. One of those reasons is due to the educational system structure.

Structure can simultaneously constrain and enhance an organization and is not easily changed (Bolman \& Deal, 2010; 2013). The organization of Missouri education is a series of smaller systems bound in larger systems full of internal and external constraints that can deter students from achieving these goals. The state level loosely follows Mintzberg's (2005) Divisionalized Form of organization (see Figure 2.2). At the top of the hierarchy in this model is the Missouri State Board of Education, and middle management and the technostructure are comprised of different departments of the DESE. The divisions that make up the operating core are the school districts in the state of Missouri to which communication can come down from every level. The idea behind the divisionalized form is to standardize the production of separate smaller organizations that have separate budgets, local control, regulations, and operations (Lunenberg, 2012; Mintzberg, 2005). In the case of Missouri education, the product is college and career ready graduates. 
At the local level, school districts loosely follow Mintzberg's (2005) model of Professional Bureaucracy (see Figure 2.3). This is a theoretical model that applies well to simple stable environments, including the one to which the Missouri education system was founded. Lunenburg (2012) provided an in-depth and simplistic adaptation of the professional bureaucracy model to contemporary school districts. In it, he described the five parts of an organization as shown in Figure 2.3, as follows:

The strategic apex (a) is top management and its support staff. In school districts, this is the superintendent of schools and the administrative cabinet. The operative core (b) are the workers who actually carry out the organization's tasks. Teachers constitute the operative core in school districts. The middle line (c) is middle- and lower-level management. Principals are the middle-level managers in school districts. The technostructure (d) are analysts such as engineers, accountants, planners, researchers, and personnel managers. In school districts, divisions such as instruction, business, personnel, public relations, research and development, and the like constitute the technostructure. The support staff (e) are the people who provide indirect services. In school districts, similar services include maintenance, clerical, food service, busing, legal counsel, and consulting to provide support. (Lunenberg, 2012, p. 2)

An argument can be made because separate schools operate in a district and follow the Divisional Form Model. However, what makes a professional bureaucracy is the staff at different schools have a shared responsibility to get as many students to graduate and become college and career ready as possible. Division of the operational core can be avoided with proper 
collaboration across building boundaries within a district, especially between feeding and receiving schools.

It is important to note that in addition to the structure of the Missouri educational system, schools are situated in an environment where many external factors inflict unique obstacles that can impede each student's success. In response, schools are becoming more of an outward agency in services they provide in order to ameliorate the outside issues that interfere with student learning. This has increased an already turbulent environment under which school districts operate. While the simple structure of the Professional Bureaucracy Model supports stable surroundings, more volatile landscapes require flexibility (Bolman \& Deal, 2013). In Missouri, schools are often positioned in simple organizational structures misaligned with their increasingly complex environments. Usually, traditional hierarchal forms of public administration are not optimal when dealing with wicked problems (Head \& Alford, 2015). In this structure, many initiatives come from the district, state or even federal levels but are carried out at the local level (Bolman \& Deal, 2010). Currently, the Missouri DESE (2020c) has a statewide initiative that supports the research-based findings of using PLC's. This is also conducive to studies that show schools that adapt external mandates to meet their contextual instead of simply conforming to them have more success (Bolman \& Deal, 2010). Schools and districts have the leeway to design a PLC structure to fit their circumstances and respond to changes in goals, technology and environment (Bolman \& Deal, 2013). Those in charge of implementing these PLC's need to be tactical in their coordination as lateral strategies need to supplement top-down regulations, protocols, and demands (Bolman \& Deal, 2013).

When conducting the PLC initiative at the local levels, leaders need to be cognizant of the needs of all the stakeholders involved. This is important because the undertaking of 


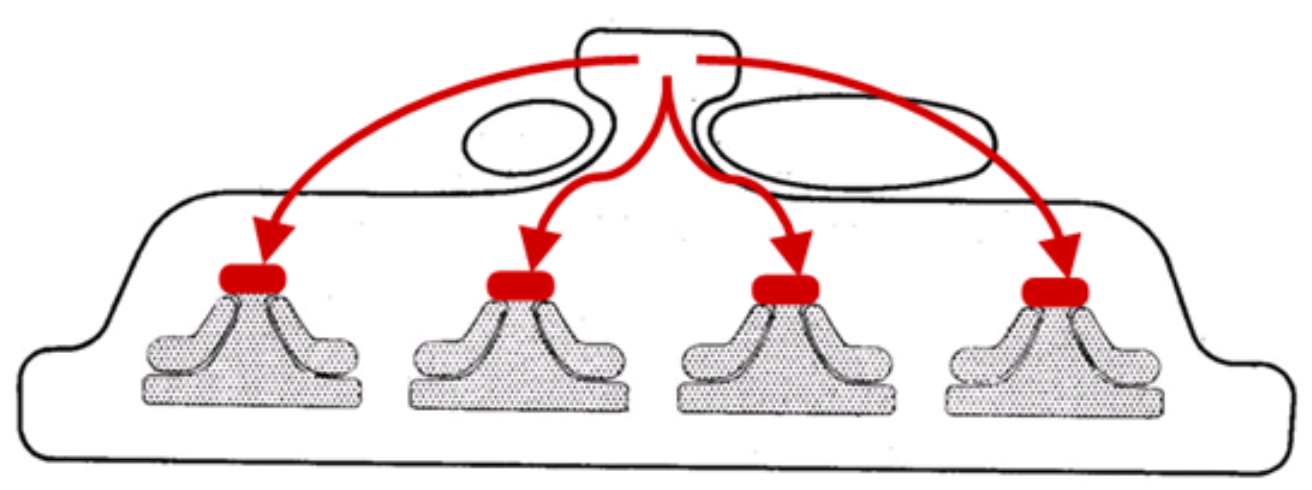

Figure 2.2. Mintzberg et al.'s (1995) Divisionalized Form Organizational Model applied to the state-level educational structure in Missouri. The arrows consist of how communication is transported throughout the state hierarchy to the administrative cabinet of each school district. Adapted from “The Divisionalized Form” by H. Mintzberg, J.B. Quinn, and J. Voyer, 1995, The Strategy Process: Collegiate Edition, 1995, p.157. Copyright 1995 by Prentice-Hall.

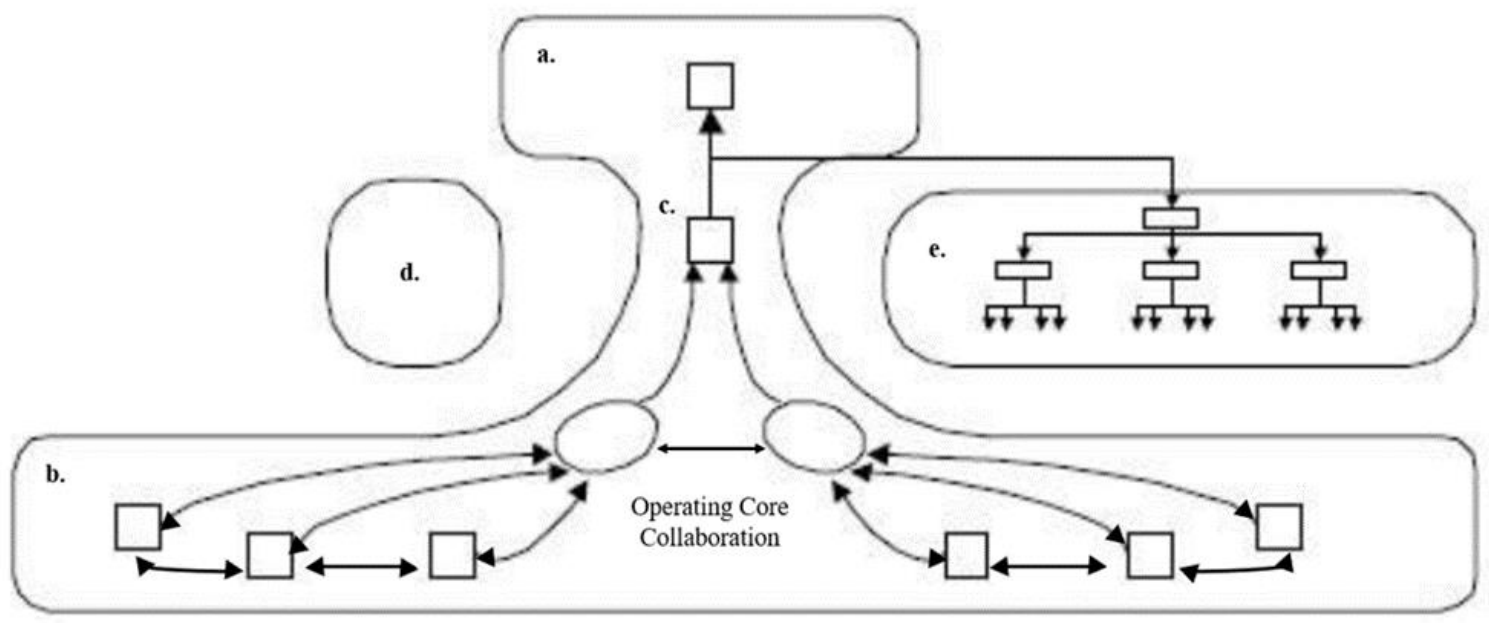

Figure 2.3. Mintzberg et al.'s (1995) Professional Bureaucracy Organizational Model applied to local school districts in the state of Missouri. The two-way arrows represent the collaboration of the teachers and other non-administrative staff in elementary and secondary schools. Adapted from "The Professional Bureaucracy" by H. Mintzberg, J.B. Quinn, and J. Voyer, 1995, The Strategy Process: Collegiate Edition, 1995, p.156. Copyright 1995 by Prentice-Hall. 
implementing PLC's is almost a wicked problem onto itself for some schools. Like striving for solutions to wicked problems, proper collaboration "entails at least some degree of shared understanding, agreed purposes, mutual trust, and usually an element of interdependence, which usually require time, effort, and skill to bring about” (Head \& Alford, 2015, p. 725). Public schools are vulnerable to external pressures due to limited resources (Bolman \& Deal, 2013) and policymakers hamstring efforts by overlaying new regulations over old ones instead of replacing them all together (Head \& Alford, 2015). Thus, it is useful to analyze them using Bolman and Deal's (2013) Political Frame along with its Structural Frame.

Districts not only enforce statewide initiatives have their own to improve their performance. That makes the environment a constant negotiation for resources such as money, people, and especially time (Bolman \& Deal, 2013). Information technologies and data sharing allow for organizations to become more efficient, flexible, and decentralized (Bolman \& Deal, 2013). However, information and data management systems are expensive to install and require time to properly train employees to be fluent in the technology, data interpretation, and communication (Datnow \& Park, 2014). Time is so limited that structurally, coordination must occur that ensures efficiency without overlapping agendas, but also exploits and rectifies gaps. Leaders also need to create coalitions of people to obtain resources and put the right people in place for objectives to be met. Unfortunately, these people can be rare in high demand situations, so others need to be recruited and trained in order to avoid an overload of responsibilities on so few (Bolman \& Deal, 2013). Since time is so insufficient for many in education, it is extremely valuable and needs to be maximized efficiently, clearly, and intentionally. 
To maintain focus and build capacity in increasingly complex organization of the statewide education system, the Missouri DESE (2020c) sustains focus of the PLC initiative universally by providing four guiding questions for participants to adhere to:

- What should students know and be able to do?

- How will the school determine students have learned the essential knowledge and skills?

- How will the school respond when students do not learn?

- How will the school respond when they already know it?

The PLC initiative and questions all align with the overarching goal of producing successful students, while its simplicity allows it to be adapted by each school district. The recommended strategy and focus also lends itself for schools to become self-sustaining learning organizations.

For schools to utilize the PLC model and become learning organizations, initial analyzation of the current structures and processes to find what works and what needs changing is imperative (Bolman \& Deal, 2013). Organizations should concurrently explore new ways to improve while exploiting methods that have already been proven to be effective and discarding those that are not (Bolman \& Deal, 2013), a process called strategic renewal (Crossan et al., 1999; Schmitt, Raisch \& Volberda, 2018). Arensman, van Waegeningh, and van Wessel (2018) suggested an idea of twinning the Theory of Change, that keeps the focus on constant change for improvement, with practices of change. Much like strategic renewal, practices of change are strategies that are carried out throughout the organization's learning process, while they continue to explore (Arensman et al., 2018). Schools cannot abandon their technical operations to focus strictly on change as it would harm current student learning. Administrators, teachers, and peripheral staff all need to maintain strategic management (Manning, 2013). Strategic management involves setting objectives, analyzing internal and external factors, evaluating 
strategies, and ensuring proper delegation of responsibility (Manning, 2013). Leaders should utilize and share data-driven tangible evidence of achieving these objectives (Bolman \& Deal, 2010), which should boost morale and motivate and reorient staff to pursue greater change.

\section{Leadership Analysis}

With the conflicting structures, unstable environment, and increasing demands on teachers and staff, leaders in the school system are integral in pursuing success in making all students college and career ready. Leaders at every level of the Missouri education system are bound by the structure and other entities when it comes to control and many major decisions are out of their hand. Their true importance could be in understanding, creating, and managing culture that produces lasting positive change from their unique position (Schein, 2005). Since educational leaders are often at the mercy of their circumstance and situations, they need to be adaptive to change and adjust their protocols and structures accordingly (Chatalalsingh \& Reeves, 2014). They also must have additional qualities that protect the well-being of their followers, empower others, and optimally manage the complex organization that engulfs them.

\section{Adaptive Leadership}

A successful learning organization has an embedded change culture that leaders need to manage. In order to do this, they need to become adaptive leaders that prepare those in their organization for change (Heifetz \& Laurie, 2010; Northouse, 2016). “Adaptive leaders engage in activities that mobilize, motivate, organize, orient and focus the attentions of others" (Northouse, 2016, p. 258). The change that Missouri educational leaders seek is performance improvement until $100 \%$ of students not only graduate but are prepared to contribute to society or pursue higher education if they so choose. This challenge is clearly defined, but within the current structure and environment, there is no direct viable solution to achieve it, and it will take the 
collective efforts of the leaders and followers to collaborate to continue in its pursuit (Heifetz \& Laurie, 2010; Northouse, 2016).

School and district leaders can become adaptive leaders by following six identified behaviors. First, educational leaders need to be able to step back and gain perspective about the direction of the school or district in which they lead, no matter how intense the struggles they may be experiencing (Heifetz \& Laurie, 2010; Northouse, 2016). Adaptive leaders can be team leaders that move fluidly between the role of authority to colleague when the situation requires it (Chatalalsingh \& Reeves, 2014); however, they share in the struggle. Leaders also need an opportunity to measure progress, analyzed data, forecast, and make informed decisions (Bolman \& Deal, 2013; Datnow \& Park, 2014). During this time, educational leaders must perform the second behavior, identify and discern adaptive challenges from technical ones (Heifetz \& Laurie, 2010; Northouse, 2016). Wicked and complex problems, like getting all students to graduate or dealing with student homelessness, are considered adaptive challenges. Technical challenges are clearly defined and can be handled with strategic management and known solutions (Manning, 2013, Northouse, 2016). For example, principals can streamline teacher efficiency by adopting new technology programs that are compatible (e.g. online gradebooks, learning platforms, email). A key element of the Path-Goal Theory is to remove obstacles (Northouse, 2016), which is not always possible with adaptive challenges. Even if principals can solve technical problems on their own, it should free up valuable time for teachers so they can focus their efforts towards adaptive challenges and change.

The third behavior of an adaptive leader is to reduce distress throughout the organization (Head \& Alford, 2015; Heifetz \& Laurie, 2010; Northouse, 2016). This is an extensive task as it takes unique skills to get others to accept change and buy into change. Leaders who try to 
change the behaviors of subordinates are often met with resistance (Schein, 2005), especially by those who are not fully invested in the shared purpose or those who feel their experience precedes the need for change and are therefore exempt from it. The democratic nature of the PLC initiative may not be well received by teachers who have individualism ingrained in their practice and philosophy (Gajda \& Koliba, 2008; Johnston \& Tsai, 2018). It also takes incredible skill for adaptive leaders to serve their followers by diagnosing and prescribing possible solutions to their problems (Northouse, 2016).

Utilizing Servant Leadership (Northouse, 2016), these leaders could also empower their people to diagnose and overcome their problems on their own through supportive training. Regardless, school administrators need to realize that those they are leading have chosen a career of service for others and suggestive change can be challenging, disorienting, and misperceived as insulting. Adaptive leaders need to tow the delicate line between getting others to challenge their beliefs by exposing them to the need of change and not overwhelming them to the point where they become unproductive (Head \& Alford, 2015; Heifetz \& Laurie, 2010; Northouse, 2016). Preceding this and all other actions of adaptive leaders is the Relational Transparency of the Authentic Leadership Model (Northouse, 2016). Teachers are unlikely to follow or trust administrators they feel are not practicing transparency to their fullest ability, whether they want to hear it or not.

Adaptive leaders also work often with teams. This is certainly true in the Missouri's PLC Initiative, as the collaboration is a key element of its implementation. However, there is a growing number of schools where creating self-managing teams are the norm to create flexibility with leadership and scheduling. In high schools, these teams may come in the form of gradelevel, departmental, academy or site-leadership teams. With self-managing teams, the external 
leader becomes a coach who gives guidance, goals, resources, and information and intervenes when it is necessary to re-orient teams (Chatalalsingh \& Reeves, 2014; Levi, 2017). Northouse (2016), stated, "Adaptive leaders understand that people need a supportive environment and adapt more successfully when they face difficult problems directly, learn to distinguish between fantasy and reality, resolve internal conflicts, and learn new attitudes or behaviors” (p. 260). They create a holding environment that is a comfortable atmosphere where people can interact honestly and the leader can regulate intensity levels (Heifetz \& Laurie, 2010; Levi, 2017; Northouse, 2016). Other responsibilities for adaptive leaders in a collaborative setting is to provide direction by reframing key questions and issues, challenge current roles on the team without changing them, and shape norms that break unproductive habits of the participants (Heifetz \& Laurie, 2010; Northouse, 2016).

Another behavior of an adaptive leader is to maintain disciplined attention (Heifetz \& Laurie, 2010; Northouse, 2016). They can reduce avoidance behaviors of participants on the team by installing activities, directions, and norms that require full participation from everyone (Levi, 2017). Another way to approach maintaining focus is to create a data driven culture. Educational leaders need to become data analysts and stewards (Datnow \& Park, 2014). As data managers, leaders need to be deciding on the problems they want solved, and the data collection and analytics should be done to fit that intention (Zettelmeyer, 2015). Educational leaders may not be experts in interpreting data, so they need to know and be able to defer data to others for analyzation when necessary. Regardless, data analysis needs to be made a priority for decisionmakers in organizations and needs to be translated appropriately to the audience it is intended (Datnow \& Park, 2014; Zettelmeyer, 2015). 
Data can help maintain focus by letting it guide collaboration and decision-making of teams (Zettelmeyer \& Bolling, 2014). The data used in education are extensive. In Missouri high schools, major statistics such as graduation rates, student grades, attendance rates, course failure rates, discipline rates, EOC and ACT scores, and formative and summative classroom and district assessments define student success. Administrators also need to make sure collaborative efforts are worthwhile. Therefore, it is imperative that a role is assigned within the PLC's to keep records, and for everyone to provide feedback on their experimentation with practice. Those statistics can make holding teachers and staff accountable to contribute and make pedagogical changes to improve student performance. However, data can also help aid against resistance by using it to create a compelling vision, earn buy-in, invest in data analytics training, and embed analytics into the daily structure of educators (Zettelmeyer \& Bolling, 2014). By being intentionally selective of data that aligns to goals and objectives, people within the school or district will have an easier time maintaining focus.

Adaptive leadership is follower centered (Northouse, 2016). Adaptive leaders empower the people within the organization by giving the work back to them, which is the fifth leadership behavior (Heifetz \& Laurie, 2010; Northouse, 2016). PLC leaders in schools need to be aware of what type and how much work teams of teachers and staff can excel at and handle (Heifetz \& Laurie, 2010). One purpose of the Missouri DESE PLC Initiative is to increase building capacity in schools (DESE, 2020c). Schools only achieve this by giving the work back to teachers for them to complete through collaboration. Collective efficacy is maximized when every participant fully invests their efforts toward the shared purpose and commits to change, organizational learning, and improvement. This leads to the final adaptive leader behavior, to protect the leadership voices in the operating core (Heifetz \& Laurie, 2010; Northouse, 2016). 
Getting input from members who are considered outsiders in a group that do not normally contribute can wreck the equilibrium of even the highest performing teams. However, the more participation a PLC has, the more knowledge, perspective, and productivity will be contributed, and school capacity will grow. The seven behaviors of adaptive leaders provide a blueprint for educational leaders that can invite the change and learning necessary to improve their school system's performance.

\section{Implications for Research in the Practitioner Setting}

Many of the educational issues facing the Missouri Education System, especially that of the high school setting are universal to every state. The dropout crisis exists in every state and Missouri is not immune. In fact, the $10.42 \%$ of students in 2019 who did not graduate in four years can potentially be extremely costly to the state's economy. Following the NCES's (2020) 2019 algorithm that stated each dropout could potentially cost society $\$ 276,000$ over the span of their lives, the estimated 27,783 potential dropouts in 2019 would drain the economy of close to 7.7 billion dollars in their lifetime. This is money that could be better allocated toward improving the schools where many of them did not successfully achieve graduation. With the wide-reaching impact of the dropout crisis, and the well-documented negative impact an unsuccessful initiation to high school can have on a student's potential to graduate, it is imperative that all districts in the state of Missouri invest serious attention toward this transition.

Schools in Missouri also face the same universal issues of scarce resources and teachers' limited time. Yet school districts recognize the impact the freshman transition can have on students and are dedicating time and resources toward implementing interventions and support programs in response. For example, Lindberg High School (Lindberg High School, 2020), St. John Vainney High School (Saint John Vainney High School, 2020), and Timberland High 
School (Timberland High School, 2020) employ the LINK Crew program to pair up freshman with upper classmen. Clayton High School (Clayton High School, 2020) and Fort Zumwalt East High School (Fort Zumwalt East High School, 2020) conduct a Freshman Transition Day. Glendale and Hillcrest High Schools of the Springfield School District (Springfield Public Schools, n.d.), the Independence School District (Independence School District, 2020), the Belton School District (Belton School District \#124, 2020), and Smith-Cotton High School (Sedalia School District 200, 2020) have all implemented the Freshman Academy Model. All these schools and districts also have a webpage designed specifically for freshman. It is almost certain that many more schools in Missouri have carried out plans to respond to the transition to high school but have not communicated it online. It is also possible that schools in Missouri have yet to intervene to ease the transition to high school for students.

The previous examples illustrate the investments of limited time, money, and resources school districts put toward responding to the freshman transition. All these efforts can be derailed by the academic inequity between feeder and receiving schools. While overwhelming research suggests to conduct collaboration between teachers across these divides (Akos \& Galassi, 2004; Chapman \& Sawyer, 2001; Dorman, 2012; Egodawatte, McDougall, \& Stoilescu, 2011; Fulk, 2003; Hazel, Pfaff, Albanes, \& Gallagher, 2014; Kowal, 2002; McCallumore \& Sparapani, 2010; Mizelle, 2005; Morgan \& Hertzog, 2001; Neild, Balfanz, \& Herzog, 2007; Oakes, 2009; Parrish et al., 2011; Schlosser, 2014; Smith, 2006a; Smith et al., 2008), none exists that identifies if districts throughout Missouri are undertaking this, nor if their efforts effect student achievement. Furthermore, the Missouri DESE's (2020) PLC Initiative make this an optimal time for this collaboration to occur. School districts now have the leeway within the 
state's PLC framework to create a community that links the teachers at each level together to focus on IPC.

Further research in this area could be extensive and extremely beneficial for school districts in the state of Missouri, especially those districts whose feeder patterns create further impediments to their students' academic progression. Schools with complex feeder systems tend to reside in heavily populated and often urban areas where supports are needed the most. Ideally, an adaptable framework that consists of effective collaborative methods across the divide can provide guidance for districts regardless of their structural make-up.

In addition, by comparing the different IPC elements effects on student achievement schools can maximize the time of their PLC's by identifying what focuses should be prioritized. A comprehensive framework of both the means and prioritized focuses of collaboration across transitions can provide guidance, clarity, and tangible solutions for leaders in charge of its coordination. They can apply the appropriate resources with assurance of its value and can hire and put the correct people in place to deal with such a unique circumstance. For any of this to occur, a discussion needs to be started between Missouri educators to build the state's capacity in dealing with the freshman transition. Through this discussion, schools can stop acting in isolation of each other and can work together to find solutions that are generalizable and adaptable to greater populations.

\section{Summary}

The academic disruption caused by the transition to high school and all its externally imposed obstacles makes it an adaptive challenge. Many events and movements throughout history have led to the current structure, regulations, and protocols used in the Missouri Education System, which contributes to the unique structural difficulties created by transitions 
from one school to another. By framing the education system at each level through Bolman and Deal's (2013) Structural and Political Frames, school districts can effectively fit their organization to their current circumstances and negotiate for the necessary resources to create change. The current PLC Initiative (DESE, 2020c) allows for the power of change to come from the operational level of schools and allows their leadership to dictate the direction they feel is best for school improvement. Leaders in these positions are best suited to adhere to the Adaptive Leadership Theory, while emphasizing organizational learning, proper data-driven guidance, and effective teamwork. School districts must make a concerted unified effort to build the state's response capacity toward the ameliorating the academic continuity through the transition to high school. By achieving this, they can turn many of the adaptive challenges created by this unique educational disruption into technical problems with achievable solutions. 
SECTION THREE: SCHOLARLY REVIEW 


\section{Introduction}

A student's academic career consists of intertwined cognitive, behavioral and relational trajectories that do not always proceed in identical directions (Benner, 2011). The transition from mid-level feeder schools to high school is a significant point in each student's career as a unique combination of those trajectories meet and can be influenced by their personal characteristics and experiences (Cohen \& Smerdon, 2009). The inability of these students to overcome this transition correlates to an increased likelihood of dropping out of high school (Alspaugh, 1998; Neild et al., 2008). Research shows the number of students who cannot adjust has contributed to a dropout crisis (Balfanz, Herzog \& Mac Iver, 2007; Chapman \& Sawyer, 2001; Ellerbrock \& Kiefer, 2014; Maynard, Salas-Wright, \& Vaughn, 2015; Neild et al., 2008). Schools have responded by targeting resources at transitional programs (McIntosh \& White, 2006; Mizelle \& Irvin, 2000; 2014; Smith, 2006a, Uvaas \& McKevitt, 2013) and school reform (Cohen \& Smerdon, 2009; Ellerbrock \& Kiefer, 2014; Emmett \& McGee, 2012; Neild, 2009). Building instructional program coherence (IPC; Newmann et al., 2001) and cross-grade collaboration is a common suggestion in research (Akos \& Galassi, 2004; Brown \& Seely, 2010; Oakes, 2009; Thayer et al., 2018) to promote academic continuity through the disruptive nature of the transition to high school. However, the research lacks if this vertical teaming between mid-level feeder schools and high schools is taking place and subsequent evidence that shows if vertical teaming influences student achievement. Finally, research fails to convey specific areas teachers should focus on during this collaboration.

The purpose of this study is to address the academic coherence between mid-level feeder school and high school teachers that will help curtail the negative effects the transition has on 
students. This review will examine the current dropout crisis and analyze the four causal domains (academic, institutional, relational, and personal) unique to the freshman transition that create insurmountable obstacles for some students to succeed. It will then connect these factors to the dropout crisis with the consequences many students face when they are unable to successfully adjust to high school. Finally, the lengths that school districts have gone to in order to compensate for this transition were examined, along with the role of collaboration and vertical teaming in creating student success in academic achievement.

\section{The Dropout Crisis}

High schools are facing a dropout crisis. Chapman et al. (2011) estimated that, depending on how and where it is measured, between 9.3 and $43.7 \%$ of freshman will fail to graduate within four years. Horwitz and Snipes (2008) found the totals to be higher at closer to $50 \%$ in their study. While some of these students still graduate, many studies have shown that approximately one-third of all freshmen will drop out of high school all together (Ellerbrock \& Kiefer, 2014; Horwitz \& Snipes, 2008). These numbers have been shown to be an underestimate for populations in certain demographics or geographic areas. In urban areas, the dropout rates of high school students have grown to approximately 50\% (Neild et al, 2008; Maynard et al., 2015) resulting in thousands. A disparity is also evident in the 4-year graduation rates for minority students as compared to their Caucasian counterparts. Johnson, Simon, and Mun (2014) found from the Alliance for Excellent Education in 2007 that $22 \%$ of white high school students did not graduate within four years. That percentage grew to $42 \%$ of Latino students, $45 \%$ of African American students, and 49\% of Native American students (Johnson et al., 2014). Socioeconomic status (SES) also contributes to high school completion rates as students who come from the bottom $25 \%$ of SES distribution are seven times more likely to drop out than 
those from the top 25\% (Johnson et al., 2014). These alarming drop out statistics have high schools focusing a lot of time and resources to pinpoint causes and come up with viable solutions to increase student success and graduation rates.

Dropping out of high school has destructive personal impacts well beyond the high school setting for students. One area is financial as dropouts are more likely to have decreased human capital (Campbell, 2015; Chapman et al., 2011; Maynard et al., 2015, Neild et al., 2008). High school becomes a crisis point for students who do not graduate as failure to receive a diploma carries substantial occupational and economic disadvantages for young adults (Neild et al., 2008, Rumberger, 2013). Compared to high school graduates, individuals who drop out are less likely to find a job with a decent wage and live above the poverty line without government assistance (Campbell, 2015; Chapman et al., 2011; McKee \& Caldarella, 2016; Rumberger 2013). For example, The National Center for Education Statistics (2018) found that:

The median earnings for full-time workers ages 25 through 34 who had not completed high school $(\$ 26,000)$ were lower than those of workers whose highest education level was high school completion $(\$ 32,000)$, an associate's degree $(\$ 39,000)$, or a bachelor's or higher degree $(\$ 55,000)$. Among 25 - to 34-year-olds in the labor force, the unemployment rate for high school dropouts (13 percent) was higher than the unemployment rate of those whose highest level of educational attainment was a high school credential (7 percent). (NCES, 2018)

Campbell (2105) argued that based off statistics, diplomas are more impactful for those who are the least likely to graduate because they have greater societal gains from receiving a diploma than those who are more likely to graduate. 
Consequences of not completing high school are not exclusive to financial and occupational gains. Those who drop out experience greater physical and mental health problems later in life than do high school graduates (Maynard et al., 2015; McKee \& Caldarella, 2016; Rumberger, 2013). In fact, graduates are about half as likely to attempt suicide in their lifetime than their dropout counterparts (Maynard et al., 2015). Yet consistent research shows that the most non-financial area of impact for dropouts is the association with incarceration rates (Bjerk, 2012; Campbell, 2015; Maynard et al., 2015; McKee \& Caldarella, 2016; Western \& Pettit, 2002). After controlling variables of race, gender, employment and income, dropout arrests were close to three times more likely than those who graduated high school (Maynard et al., 2015). Western and Pettit (2002) conducted a study that showed in 2000, black male high school dropouts more likely to be incarcerated than employed.

The dropout crisis has an expensive impact on society as well. Due to lower tax contributions, higher reliance on government assistance programs, and higher criminal incidence rates, each dropout has an additional lifelong cost to the economy of approximately $\$ 276,000$ (NCES, 2020). In fact, following the same criteria, Maynard et al. (2015) calculated the dropout respondents in their study (approximately 1,931 emerging adults) would amass a cost of over $\$ 500$ million in their lifetime to society. U.S. taxpayers would recoup an estimated $\$ 45$ billion annually if the dropout rate was reduced by 50\% (Cavendish, 2013; Levin et al., 2007). However, there is a variation in the success and contributions of different types of high school dropouts. The idea of Push Theory states students can be "pushed out" due to academic, behavioral and school related contextual causes, or "pulled out" due to reasons like taking care of family or health reasons (Bjerk, 2012; Campbell, 2015). Students who are "pulled out" have similar outcomes regarding the labor market and criminal activity as their graduate counterparts, 
mainly due to immediately joining the workforce once they leave school (Bjerk, 2012). In contrast, students who are "pushed out" tend to remain idle after leaving and therefore perform substantially worse in gaining human capital and avoiding incarceration than their graduate counterparts (Bjerk, 2012). The contrast illustrates why schools need to determine their responsibility in the graduation rate, and what they can do to increase it.

Benner (2011) used the Life Course Theory to convey that while dropping out of school is a measurable event, it is caused by a pattern that unfolds over time. The pathways for many dropouts show a persistent pattern in grade retention, absenteeism, and behavior problems all the way back to kindergarten (Hickman, Bartholomew, Mathwig, \& Heinrich, 2008). This is evident by the number of dropout predictors found from mid-level feeder schools (Akos \& Galassi, 2004; Casillas et al., 2012; McKee \& Caldarella, 2016; Neild, 2009; Neild et al., 2007). These predictors include poor grades in core subjects, low attendance, failure to be promoted to the next grade, disengagement in the classroom, and behavioral problems (Kennelly \& Monrad, 2007). The sixth grade is the focus of many studies as it is the first major indicative predictor of dropping out and is the natural transition for many students to a new school (Balfanz, 2011; McCallumore \& Sparapani, 2010; Neild et al., 2007). Studies in Philadelphia show that sixth graders had a $75 \%$ greater risk of dropping out if they had failed math or English, had attendance below $80 \%$, or at least one negative behavior incident during the year (McCallumore \& Sparapani 2010; Neild et al., 2007). Balfanz et al. (2007) added that from sixth grade through eighth grade, students from high-poverty urban areas that identify with more than one of those same indicators in the same year have a $29 \%$ graduation rate.

While all grades contribute to students dropping out, there is significant association with ninth grade course failure (Allensworth \& Easton, 2007; Alspaugh, 1998; Hazel et al., 2014; 
Roybal et al., 2014) regardless of demographics, previous performance, or school attitude and ambition (Neild et al., 2008). Academic success throughout the first year of high school increases the probability that students graduate, even for those who transfer or leave school for an extended period (Rosen, Warkentien, \& Rotermund, 2019). However, extensive findings show adolescents' achievement level consistently declines across this transition (Alspaugh, 1998; Benner, 2011; Cohen \& Smerdon, 2009; Kennelly \& Monrad, 2007; Neild et al., 2008; Smith et al., 2008; Somers, Owens, \& Piliawasky, 2009). Students' first year grade point average (GPA) in high school is a predictor of the rigor level of their high school course path as well as their overall cumulative GPA (Langenkamp, 2009). Course failures, absenteeism, and behavioral problems increase and test scores decline more severely in the ninth grade than any other grade level (Chapman \& Sawyer, 2001; Cohen \& Smerdon, 2006; Smith, 2006; Smith et al., 2008).

An overwhelming amount of high schools have their highest enrollment in the ninth grade due to transfers failure of promotion to the tenth grade (Cohen \& Smerdon, 2009; Emmet \& McGee, 2012). However, these schools tend to hemorrhage students to drop out, largely by the end of the freshman year (Cohen \& Smerdon, 2009; Emmet \& McGee, 2012). Approximately 25\% students entering the ninth grade, and exceeding 50\% in certain communities, will fail to receive a diploma (Casillas et al., 2012). Approximately $6 \%$ of all dropouts leave before they begin their sophomore year (Ellerbrock \& Kiefer, 2014; Mizelle, 1999). There is a statistically significant moderate negative relationship between poverty and graduation rate of students (Baydu, Kaplan, \& Bayar, 2013), which contributes to higher dropout rates in urban areas. Neild et al.' s (2008) study in urban high schools illustrated the consequence of this as $60 \%$ of their participants who were not promoted within their first year of 
high school dropped out within 6 years, while just $8 \%$ graduated (Neild et al., 2008). Bornsheuer et al. (2018) found that students in a Southeast Texas high school retained in the ninth grade were approximately six times less likely to graduate on time (p. 14). Echoing this finding was a study done by Pharris-Ciurej, Hirschman, and Willhoft (2012), who found that failure numbers in high school due to retention and attrition, almost mirrored the numbers of those who graduated high school within four years of entrance.

Disruptions in the patterns of a student's academic progression can also make them vulnerable to dropping out of high school. In fact, Dupéré et al. (2018) found that approximately $40 \%$ of students who dropped out did so when they were recently exposed to at least one event considered moderately or severely stressful. The transition to high school could be considered a stressful event, as it is a key point when academic, contextual, social, and personal trajectories meet and make it difficult for students to overcome its trials and emotional strain (Cohen \& Smerdon; 2009; Gentle-Genitty, 2009). Youngman (1986) stated that in 1986, 10\% of British students suffer serious problems after they enter high school. By comparison, in the United States in 2004, that number had increased to $40 \%$ (Akos \& Galassi, 2004). Students' selfperception and confidence in their completion of high school is imperative in dealing with these stressors (Rosen et al., 2019). This is especially true since it is during the transition to high school that the student narrative starts to associate perceived life chances with school success (Chapman \& Sawyer, 2001). For students who previously struggled and feel that success is unattainable, the connectedness to school is already threatened before they even really begin high school (Chapman \& Sawyer, 2001). This troubling trend has researchers examining what academic, institutional, social, and personal factors all contribute to an incoming freshman's 
first-year success or failure, as it can set the tone for the rest of their academic path and beyond (McIntosh \& White, 2006).

\section{Causal Transition Factors}

The risk ninth grade students face throughout their transition from mid-level feeder schools is well established (Somers et al., 2009). While individual experiences vary through the transition, common educational, institutional, social, and personal factors contribute to create stress and obstacles that put these students in academic jeopardy (Galla, Tsukayama, Park, Yu. \& Duckworth, 2020). Students' ability to overcome these factors can lead to success, while failure can result in an increased likelihood of dropping out of school. The following sections outline those causal academic, institutional, social, and personal factors that converge to make the freshman experience so unique.

\section{Academic Factors}

As previously determined, the disruption of the transition to high school has an almost universal negative effect on student academic achievement (Alspaugh, 1998; Benner, 2011; Cohen \& Smerdon, 2009; Kennelly \& Monrad, 2007; Neild et al., 2008; Smith et al., 2008). The academic factors most associated with this decline are the credit accumulating requirements coupled with the increased rigor students experience when entering high school.

Credit Accumulation. An immediate stressor for students entering high school is that, because the goal is graduation, they start to attribute future opportunities outside of school to academic success in school (Chapman \& Sawyer, 2001). Part of this is because state-mandated high school requirements differ from those in mid-level feeder schools and include accrual of successful course completion for graduation (McCallumore \& Sparapani, 2010). Therefore, 
students enter a credit accumulation requirement system, where for the first time in their academic careers they must earn passing grades in core courses to advance (Emmett \& McGee, 2012; Fulk, 2003; McCallumore \& Sparapani, 2010; Neild et al., 2008, Smith et al., 2008). Many students are unaware of the newly required course success as it is different from the promotion methods in their mid-level feeder school where they learned a system to just "get by" (Neild, 2009). Distracted with acclimating to the environment and adjusting to the higher rigor, they usually realize the importance of passing their freshman courses after that opportunity is gone (McCallumore \& Sparapani, 2010; Neild et al. 2008; Smith et al., 2008). What makes it more difficult is it can take a year for students to recover to the achievement level they were at before the transition (Akos \& Galassi, 2004; Alspaugh, 2001; Somers et al., 2009; Uvaas \& McKevitt, 2013). For those students, their reality becomes that pursuing their chosen career path is much more difficult because necessary course completion, skills, and knowledge are much harder to obtain (Cooper \& Liou, 2007). This is not due to a lack of intellect or motivation, but rather their inability to successfully navigate and negotiate the educational system (Cooper \& Liou, 2007). Not realizing the importance of classroom success early enough is one reason why course failure and dropout rates are higher in the ninth grade than any other grade (Smith et al., 2008).

When students do not earn enough credits for promotion in the allotted time, they go into credit recovery mode, or they are "off track" (Neild, 2009). Failed courses must be retaken, and promotion to the next grade, and ultimately graduation, will be deferred until credit requirements are met (Neild, 2009; Mizelle \& Irvin, 2000; Smith et al., 2008; Uvaas \& McKevitt, 2013). That is why course failure is a better indicator of dropping out than low test scores from mid-level feeder schools (Balfanz et al., 2007), and why it is magnified during the freshman year (McKee 
\& Caldarella, 2016; Roybal et al., 2014). Deficient credit accrual creates an enrollment bulge in ninth grade followed by a bottleneck before the tenth grade as students either dropout or remain freshman until they earn enough credits (Cohen \& Smerdon, 2009; Emmett \& McGee, 2012). Data show that once students successfully reach tenth grade, they are likely to graduate high school (McCallumore \& Sparapani, 2010). Allensworth and Easton (2005) found that $81 \%$ of Chicago Public School students that ended their freshman year on track graduated within four years with an additional $4 \%$ finishing in their fifth. Seeing that this relationship did not change when additional factors such as race, gender, SES, and prior achievement were considered, the correlation between being on track at the end of the first year of high school and graduation rates is very strong (Allensworth \& Easton, 2005). Ultimately, success or failure in the ninth grade sets the tone for the rest of high school (McIntosh \& White, 2006).

Increased high school rigor. A noticeable disparity in rigor exists between high school and mid-level feeder school classes for both high and low achieving students (McCallumore \& Sparapani, 2010). Three out of four freshmen report their classes are much more difficult than their mid-level feeder school classes (Chapman \& Sawyer, 2001). Parents and students are aware of the rigor increase during both middle and high school as they state academic success and increased demands (e.g. homework) as a top concern (Akos \& Galassi, 2004; Uvaas \& McKevitt, 2013; Wentzel, et al., 2019). Ninth graders report they are held to higher standards and expectations by their teachers through larger workloads and increased demand for their time to be spent on independent studying and learning (Galla et al., 2020; Mizelle, 2005). They also report more severe consequences for not completing work in high school than in mid-level feeder school (Ellerbrock \& Kiefer, 2013). 
In addition to the rigorous content, the class structure is more difficult for students as they go from a communal setting in their mid-level feeder school to a more demanding bureaucratic environment in high school (Ellerbrock \& Kiefer, 2013; Neild et al. 2008; Neild, 2009). In this change, subjects are seen by students to be more disconnected and discrete than they were in earlier grade levels (Ganeson \& Enrich, 2009). Overall, the inequity in rigor along with the well documented decline in achievement scores across the transition to high school does not allow students the access to educational opportunities due to their academic stumbles as freshmen (Alspaugh, 1998; Benner, 2011; Cohen \& Smerdon, 2009). This often leads to an examination of the preparation students receive in mid-level feeder schools to be successful in high school.

Credit accumulation makes students' perceptions of goals in high school more performance oriented and less mastery oriented than it was in their mid-level feeder school (Anderman \& Midgley, 2004). Unfortunately, students who earned decent subjective grades but were often unchallenged and those who were at-risk of failure, regularly come to high school unprepared and their lack of skills and mastery knowledge finally catches up with them (Neild et al., 2007; Neild, 2009). As Emmet and McGee (2012) pointed out, "Without the foundational skills, access to academic content becomes limited and the credit accrual system that drives the process of high school graduation becomes a difficult obstacle to overcome" (p. 75). According to the research of Cooney and Bottoms (2003), 51\% of high school teachers feel incoming students do not possess the necessary background knowledge when they enter high school. Struggling freshmen often come to high school with weak math and reading skills yet are expected to pass college preparatory classes they see for the first time (Brown \& Seely, 2010; Neild \& Balfanz, 2006; Neild, 2009). 
Neild (2009) illustrated the lack of preparation problem through the 2008 National Mathematics Advisory Panel recommendation that Algebra students need to have previously acquired mastery of fractions, decimals, and integers; however, many do not. Students without those skills may not be able to acquire them from their Algebra course due to the already limited time to complete the required curriculum (Cohen \& Smerdon, 2009), and school teachers may also lack the expertise to teach content for the earlier grades (Neild, 2009). The predicament for high school teachers is that new reforms repeatedly call for more rigor (Cohen \& Smerdon, 2009). But Corcoran and Silander (2009) contended, "at the same time that high schools are being asked to offer more rigorous preparation for larger numbers of students, they are also being asked to ensure that all or almost all students meet rising academic standards and that dropout rates decline" (p. 158). This demonstrates why higher eighth grade achievement scores were predictive in freshmen success (McKee \& Caldarella, 2016; Neild, 2009), graduating on time (Lovelace et al., 2017), and college readiness (Huerta, Watt \& Butcher, 2013).

A wide-spread suggestion in research is for mid-level feeder schools to increase the rigor in their curriculum and incorporate classroom practices that will prepare students for the learning environment they will experience in high school (Chapman \& Sawyer, 2001; Cohen \& Smerdon, 2009; Huerta et al., 2013; McCallumore \& Sparapani, 2010; Smith, 2006). Neild (2009) agreed and stated, "the problem lies with students' weak preparation for high-school work or misconceptions of the diligence required to earn a high school diploma, or both" (pp. 59-60). Academic press is major predictive concept of student effort and academic achievement as academic and behavioral problems students have in mid-level feeder schools do not self-correct, especially in high-poverty populations (Balfanz et al., 2007). Mid-level feeder schools should 
prepare both struggling students (Parrish et al., 2011) and those set for advanced courses in ninth grade (Cooney \& Bottoms, 2003) the same way, by providing extra help and adult mentoring.

When preparing students to be successful in high school, teachers at the lower grade levels need to shoulder some of the load. Neild (2009) went as far as saying that students' weak preparation in mid-level feeder schools is the primary culprit for ninth grade difficulty as evident by the number of inconsistencies of the scores for both high and low achieving middle and high school students. Mid-level feeder schools should provide a challenging and rigorous curriculum that provides the necessary mastery knowledge for students to successfully build upon in high school even beyond the ninth grade (Cohen \& Smerdon, 2009; Mizelle, 1995; Nelson, Fairchild, Grossenbacher, \& Landers, 2007; Parrish et al., 2011; Smith, 2006a). However, content should not be the only focus of mid-level feeder schools in preparing students for high school and could be detrimental to many students (Dedmond, Brown \& LaFauci, 2006).

Effective mid-level feeder school teachers are intentional and take great pride in promoting well-equipped students to high school by setting and enforcing high classroom standards and expectations that promote independence and responsibility of students in their learning (Cooney \& Bottoms, 2003; Mizelle, 2005; Parrish et al., 2011). Additional avenues of high school preparation include ample communication that provides both students and parents with information and awareness of both high school and college expectations (Parrish et al., 2011), and implementing intervention programs that support and build students confidence in their meta-learning (Cohen \& Smerdon, 2009). Cauley and Jovanovich (2006) reported that ninth graders agreed stating, "they would have been better prepared for high school had their middle school teachers challenged them more, held them responsible for their learning, and taught them strategies to learn on their own" (pp. 17-18). The bottom line is that it is essential 
for mid-level feeder school teachers to prepare students for the rigors of high school in every academic facet they can (McCallumore \& Sparapani, 2010).

\section{Institutional Factors}

For many students, the educational process consists of differing contextual and geographic settings in their educational institution as they advance. Their unfamiliarity with these settings contributes to the disruptions they face in their academic progression. These institutional factors include both district pathways and the structure and context of their schools.

District pathways. The sequential path students take can affect their academic performance. The more transitions a student makes from elementary negatively associates with achievement, resulting in a higher dropout rate (Alspaugh, 2001; Felmee, McMillan, Inara Rodis, \& Osgood, 2018). Also, students going through a multiple feeder system without a transition program in place exhibit significantly lower levels of social integration than their single feeder counterparts (Felmee et al., 2018). Students in multiple-feeder districts have the greatest odds of having the largest decrease in achievement upon entering high school. This is averse to singlefeeder districts who show the opposite pattern and the odds for success are higher after the transition (Felmee et al., 2018). Langenkamp (2009) contended that mixed social integration throughout the pathway yields higher student achievement than uniform social integration that occurs in single-feeder systems. However, too much divergence in a transition can cause isolation for students and achievement may be threatened unless offered additional school support (Langenkamp, 2009).

Structure and context. Upon arriving to high school students must navigate a larger school with a new layout and a greater, more diverse population (Felmee et al., 2018, GentleGenitty, 2009, Oakes, 2009, Uvaas \& McKevitt, 2013). In addition, they must adapt to new 
schedules, class expectations and procedures through developing skills like organization and time management (Uvaas \& McKevitt, 2013). High school is also more competitive and goal oriented, as the number of curricular and non-curricular opportunities increase (Mizelle \& Irvin, 2000). However, the new landscape may not fit the students' social, emotional or intellectual needs (Wentzel et al., 2018). The larger size and hurried climate of the school can make a student feel overwhelmed, anonymous and alienated, resulting in having insufficient time with teachers and other support sources (Ellerbrock \& Kiefer, 2013, Neild, 2009, Uvaas \& McKevitt, 2013). Organizational and procedural changes were among the top high school concerns for both students and parents (Akos \& Galassi, 2004). The sooner freshmen adjust to the structure, the sooner they can improve academically and socially (Akos \& Galassi, 2004, Langenkamp, 2009).

\section{Social Factors}

While students are acclimating to a new environment, different procedures, required credit accumulation, and the daunting rigor of high school, they are simultaneously reorienting their social world as well. Social convoys and prominent mentors can be extremely influential on how students adjust to high school. The following outlines the role peers and teachers play throughout the transition.

Peers. A top attraction for incoming freshmen is making new friends (Akos \& Galassi, 2004). By doing so they can replenish their reputations and distance themselves from detrimental peer groups (Langenkamp, 2009). Social convoys are broken with the transition (Benner, 2011; Cohen \& Smerdon, 2009; Neild, 2009), and students have to adjust to new friends, classmates, and the school's social expectations (Uvaas \& McKevitt, 2013). The decline in regular contact with former friends can make it difficult to assimilate, and the busy 
schedule may not permit enough time for new peers to evolve into perceived friends (Felmee et al., 2018). Losing friends from previous school levels is often unanticipated and disappointing to students (Ganeson \& Enrich, 2009) and can have detrimental consequences. While even having one supportive friend makes the freshman experience and high school seem survivable (Ganeson \& Enrich, 2009), perceived declining classmate support stimulates decreased mental health (De Wit et al., 2011). As Oakes (2009) stated, "Without the proper information and support, incoming ninth graders can perceive high school as an impersonal and unsupportive place and turn to unconstructive behaviors to find fulfillment" (p. 1). Forging or maintaining positive peer relationships eases the emotional burden assimilating to the new school engenders (Benner, Boyle \& Bakhtiari, 2017).

Guiding social relationships through the transition can have varying influence on academic achievement (Langenkamp, 2009, 2011; Roybal et al., 2014). Mid-level feeder school popularity has a positive association with social integration, course placement, and achievement after the transition to high school (Benner, 2011; Langenkamp, 2011). The converse of this is true suggesting that a social and academic decline create a downward reciprocal dynamic that can linger through the rest of high school (Felmee et al., 2018). When academic values and beliefs are challenged by struggles, students are more susceptible to have their needs gratified by peers and social desirability through this transition. (Benner, 2011, Gentle-Genitty, 2009).

Teachers. The teacher-student relationship is a pivotal influence on a student' $\mathrm{s}$ ability to adapt to high school. Mid-level feeder school students with positive teacher relationships tend to earn higher grades after entering high school (Benner, 2011; Langenkamp 2009, 2011). The transition provides students in a feeder program without those teacher relationships a fresh start by potentially breaking away from a seemingly negative reputation (Langenkamp, 2009), but 
also breaks the social bond they had with their former teachers (Neild, 2009). High school teachers can provide a bridging sense of belonging to students (Roybal et al., 2014). This is a skill that should be specifically emphasized by ninth grade teachers recognizing all the challenges outside of academics plaguing newly enrolled students (Ganeson \& Ehrich, 2009; Newman et al., 2007). These teachers need to find a special way to instill higher academic standards unforeseen to most of their students, while connecting school to their future (De Wit et al., 2011; Neild, 2009) and showing an emotional investment to the students' lives in a more peripheral way (Newman et al., 2007). They can start by not imposing unnecessary authoritative control (Newman et al., 2007), being empathetic, thorough, and approachable (Butts \& Cruzeiro, 2005), and not rushing the students' adjustment to high school (Ganeson \& Ehrich, 2009). In recognition, schools have gone as far as to identify teachers who excel in these skills and characteristics and restructuring their staff around them (Parrish et al., 2011).

Even with the efforts made by high schools to forge meaningful and effective studentteacher relationships, an unfamiliar setting increases the degree of difficulty that they occur. Due to the disjoint, time-constricted, non-communal structure that is foreign to mid-level feeder schools, high school teachers can often be perceived by freshman as inaccessible (Ganeson \& Ehrich, 2009; Mizelle, 2005). Yet in that new environment, having an adult figure at school playing an adaptive role by promoting both academic competency and emotional well-being throughout the transition can substantially ease its disruptiveness and improve student success (Allensworth \& Easton, 2007; Hazel et al., 2014; Wentzel et al., 2019). Teachers offering more individual attention to help with personal problems, along with emphasizing expectations for a greater work ethic and higher future aspirations resulted in fewer course failures for students (Neild, 2009). Paradoxical to this idea is De Wit et al.' s (2011) findings that showed a positive 
association between teacher support and social anxiety. However, further findings suggested this was due to teachers generating students' awareness for the need to make sense of their world, overcome their fears, and start planning for their future and current needs (De Wit et al., 2011).

Teachers fulfill specific needs of transitioning students that supportive peers, parents, and other staff simply cannot (DeWit et al., 2011). Trust in a teacher is built when they are shown as being a resource beyond classroom content (Brake, 2019). The sheer interaction time alone with teachers forces students, especially for learning disabled students (Letrello \& Miles, 2003), to rely on them the most to help adjust. One way to capitalize on that interaction is to frequently provide crucial information to freshmen that will help them properly adjust (Cooper \& Liou, 2007). Much of the time, that responsibility is disproportionately put on a small number of school counselors, which, due to their limited interaction with much of the student body, creates a constraint to high stakes information to which freshmen need access to (Cooper \& Liou, 2007).

Another way teachers can utilize their time to help freshman is through producing an effective academic climate in their classes. An effective academic climate requires teachers to practice emotional intelligence on how their instruction, activities, assignments, and interactions are perceived by students (Brake, 2019). In Butts and Cruzeiro's (2005) study, the ninth graders they surveyed stated the greatest positive influence on their transition to high school was having class engagement that made them want to attend. Student engagement has been known to have a correlation with academic performance (Newman et al., 2007) and creating trust in the teacher (Brake, 2019). Additional teacher techniques that have positive impacts on transitioning students are to communicate and hold students accountable to high academic standards (Allensworth \& Easton, 2007) and offer focused intentional remediation for students when they struggle (Parrish 
et al., 2011). Perceived academic negligence on the teacher' s part can derail any potential relationship or avenue of support for students.

Teachers are viewed by students as institutional agents whose positive relationships can support the most change (Langenkamp, 2009). This makes teacher efficacy important. Eccles and Roeser (2011) broadened previous research of teacher efficacy to include teacher optimism, defining it as the, "confidence in one' s ability to teach the students and in students' ability to learn and master demanding material and trust of both students and parents" (p. 227). Under this definition, it is teacher optimism that drives effective teaching and effective teacher-student relationships (Eccles \& Roeser, 2011). Optimism can also be influenced positively and negatively by school characteristics (Eccles \& Roeser, 2011). Larger high schools offer students positive teacher-relationship building opportunities with a broader selection of teachers to connect with through multiple curricular and extra-curricular activities, coupled with consistent collective adult monitoring of their progress (Eccles \& Roeser, 2011). However, the proportion of teachers with positive self-efficacy steadily declines from elementary through high school due to the increasingly demanding environments (Eccles \& Roeser, 2011). Teaching a rigorous curriculum to more students in a shorter allotted time makes it more difficult for high school teachers to connect with more students in the classroom setting. In high school, students can perceive teachers are less attentive to their needs due to seeing a greater number of teachers with diverse teaching styles (Wentzel, et al., 2019). This can leave students feeling as though they are interacting with authority instead of connecting with support (Chapman \& Sawyer, 2001).

Like peer and parent relationships, negatively perceived relationships with teachers often leads to more disruptions for students across the high school transition (Benner, 2011). However, the disruption and likelihood of depression decrease across the transition for students 
who view their teachers as supportive (Benner, 2011; Wentzel et al., 2019). Due to the positive effect on academic engagement, and performance during adolescence, Wentzel et al. (2019) suggested the primary goal for high school teachers should be establishing meaningful relationships with their students.

\section{Personal Factors}

While students face a lot of external influences that effect their transition to high school, they also have personal characteristics that do as well. Some of these characteristics are demographic traits that are inherent, and some are brought on by the changes that occur during adolescence. Each student's personal composition and growth contributes to his or her unique transition experience and ability to adjust.

Demographics. Overall, the transition to high school has negative consequences on all populations but is magnified across all demographic subpopulations (Neild, 2009; Uvaas \& McKevitt, 2013). The effects of the transition mirror those of dropout rates and academic deficiencies shown in urban areas (Finn, 2006). The findings from a survey administered by Benner and Graham (2009) to a diverse sample of 2000 transitioning students showed an increase in anxiety, loneliness, and declining academic performance consistent across all ethnicities. Yet, research shows significant differential effects on students based on gender, race, nativity, and socioeconomic status (Cohen \& Smerdon, 2009). For example, a student' s SES associates with dropping out (Neild et al., 2008; Neild 2009), and up to $40 \%$ of students drop out after ninth grade in schools largely populated by low-income students (Cohen \& Smerdon, 2009). As for gender, boys experience increased depressive symptoms across the transition, 
while girls experience an inverse pattern (Benner et al., 2017). The paradoxical pattern was paralleled for students classified based on birthplace. A study done by Benner et al. (2017) showed the loneliness of foreign-born citizens decreased while entering high school, while USborn students' loneliness increased. The opposite pattern emerged with school engagement.

Pertaining to race, the effects of the achievement gap along with individual, peer family, community, and school risk factors, make low-income African American students the most susceptible to transitional problems (Gentle-Genitty, 2009), especially for those with parents having a lower educational background (Langenkamp, 2009). These problems vary among different racial subgroups as well. In a comprehensive comparative study between race, gender, and previous performance, Sutton, Langenkamp, Muller, and Schiller (2018) found that only top performing white female students improved through the transition. By striking comparison, the two groups whose achievement was negatively impacted the most were high-achieving young black men and high-achieving Latina students (Sutton et al., 2018). The troubling achievement gap growth for at-risk populations found in research has school districts perplexed at finding solutions. Defying that trend at the time was Davis, Chang, Andrzejewski, \& Poirier (2014), who found interdisciplinary teaming in small learning communities was closing the gaps in relationship quality across the divide for at-risk populations. While some progress has been made, the growing achievement gap for diverse subpopulations across the transition to high school remains a substantial and prevalent problem in education today.

Age. Coinciding with the transition to high school are the personal life-course changes that occur for adolescents at this age (Neild, 2009). Many students are hitting puberty and experiencing the physical, psychological, and cognitive changes it manifests (Gentle-Genitty, 2009). Potential stress and anxiety can stem from a student's emerging cognitive capacity when 
they struggle to adjust to high school (Cohen \& Smerdon, 2009; Wentzel et al., 2019).

Demanding school experiences and decline in academic performance can lead to students increasingly questioning their competence (Cohen \& Smerdon, 2009; Wentzel et al., 2019).

These transitional elements also converge at an age where most students experience increased autonomy (Cohen \& Smerdon, 2009; Chapman \& Sawyer, 2001; Neild, 2009). Their roles become more confusing as they are still dependent on parents and teachers but have increased independence in many of their decisions (Gentle-Genitty, 2009). While students appreciate the increased freedom, they do not always realize the gravity of their academic (Cohen \& Smerdon, 2009; Smith et al., 2008) or behavioral choices (Cohen \& Smerdon, 2009; Neild et al., 2008). As adolescents reprioritize the influence of parents and peers, they exhibit increased risk-taking behaviors (e.g., skipping class) and a consequent decline in academic performance (Neild et al., 2008; Neild, 2009). While positive external support can be very beneficial in guiding students through the transition to high school (Newman et al. 2007), failure to properly navigate their increased independence can instantly derail their progress.

\section{Consequences of Transitional Factors}

The transition into high school is comprised of many different academic, structural, social and personal factors that converge to make the assimilation process difficult for students to overcome. Failing to properly integrate to the high school setting can create psychological, relational, and behavioral consequences that can alter a student's trajectory and increase the likelihood they drop out of high school. The resulting effects are often intermingled and unique to each student, requiring complex responses from schools to keep students on track for graduation. The following summarizes the consequences from the freshman transition factors that can ultimately determine if a student graduates or not. 


\section{Psychological Consequences}

When students reach high school, many factors converge to create a disorienting and often negative atmosphere they have trouble overcoming. Their lack of ability to assimilate to their new environment often has psychological consequences that can lead to even worse outcomes. The following outlines the research on the two main psychological effects the freshman transition can have on students: anxiety and depression, and self-perception issues.

Anxiety and depression. While the Life Course Theory suggests that high school dropouts result in a repeated pattern of events over an extended period of time (Benner, 2011), there exists a "9th grade shock" that effects each student in different ways (Pharris-Ciurej et al., 2012). The uncertainty caused by transitions can impose a disorienting effect that drains students' psychological assets as they try to reacclimate (Benner, 2011). During this time, as they try to reorient their lives, students become increasing susceptible to anxiety (Cauley \& Jovanovich, 2006) and depression (Newman et al., 2007). One reason students have difficulty coping is the number of stressors they encounter their first year of high school. The severity of these stressors can directly correlate to students dropping out (Dupéré et al., 2018). Any high school student exposed to severe acute stressors is vulnerable to leaving school regardless of previous performance (Dupéré et al., 2018). One way these stressors can lead to anxiety or depression is due to their effect on academics.

Part of the shock most students experience upon entering high school is the unexpected decline in grades and performance (Pharris-Ciurej et al., 2012; Suldo et al., 2009; Suldo \& Shaunessy-Dedrick, 2013; Uvaas \& McKevitt, 2013). Students GPA declines with increased exposure to stressors (Uvaas \& McKevitt, 2013), which could compound dropout risk at an earlier age for those already at risk due to previous trauma or academic and behavioral issues 
(Dupéré et al., 2018). Increased daily stressors have been shown to result in students having lower academic standards and showing less effort in independent work and school preparation (Uvaas \& McKevitt, 2013). Teachers in this context should foster perseverance when students are triggered by academic challenges and subsequent anxiety (Martin, 2011). Students in that situation often develop attentional biases towards school-related cues that they consider threatening, which is a significant predictor of not completing high school (Hickman et al., 2017). Stressors usually reach their peak for most student around the middle of their freshman year but decrease beyond that point (Roybal et al., 2014). Dupéré et al. (2018) agreed but found that the fourth quarter was the only quarter of the year significantly associated with increased dropout rates for students exposed to their highest amount of moderate or severe stressors. This could be attributed to students who realize too late they are deficient in credit accumulation to be promoted. These students, floundering academically and questioning their ability to graduate, succumb to more overt negative external actions that lead to dropping out (Neild, 2009).

However, it is not just students at grade-level or at risk that experience stressors; students in accelerated courses experience a substantial increase in stress when entering high school (Suldo et al., 2009; Suldo \& Shaunessy-Dedrick, 2013). While their general education peers have more of a variety of influences responsible for the stressors they encounter, accelerated students tend to focus on academic pressure (Suldo et al., 2009; Suldo \& Shaunessy-Dedrick, 2013). Generally, these accelerated students overcome these stressors and adapt quicker for two reasons. The first reason is this academic pressure creates a eustress that provides motivation and facilitates effective action for them to succeed (Suldo et al., 2009). The second reason is these students often have another method of coping through a social support system consisting of parents and peers. 
As students enter high school perceived loss of external support from friends and parents can lead to a lack of a sense of belonging and subsequent depression (Benner \& Graham, 2009, Benner et al., 2011, Newman et al., 2007). Research has illustrated that transition results in an increase in loneliness in students (Benner et al., 2011; Chapman \& Sawyer, 2001; GentleGenitty, 2009) that continues to increase for minority subgroups in their first two years of high school (Benner \& Graham, 2009). Newman et al. (2007) illustrated that when gender, academic achievement, and other life stressors were controlled, peer support, parent support, and school belonging were significantly associated with depressive symptoms. Parent support was shown to be most crucial as it positively associated the student's self-image, self-esteem, self-confidence, mental health, and academic success (Newman et al., 2007). De Wit et al. (2011) echoed these results, finding a statistically significant positive association between self-esteem and social support, and a statistically significant negative association between social support and both depression and social anxiety. In other words, "when support from parents or friends increased, stressors decreased and school membership increased" (Roybal et al., 2014, p. 475).

Schools and staff need to implement timely and strategic interventions for students experiencing anxiety and depression to help them cope and avoid abandoning school all together (Dupéré et al., 2018). Adolescents who develop a sufficient capacity to negotiate difficult obstacles using stable, nonreactive means are better equipped to cope with the overwhelming problems in their lives (Galla et al., 2020). They exhibit confidence which increases their responsibility, participation, and school belonging while reducing their self-handicapping and disengaging tendencies (Martin, 2011). When students feel confident, they are also able to successfully adapt to high school faster, which results in higher academic achievement (Ganeson \& Ehrich, 2009). They also exhibit courage to combat feelings of helplessness and avoidance 
behaviors (Martin, 2011). Overall, the research dictates that, "at a minimum, the transition to high school be given careful consideration when timing mindfulness-based interventions" (Galla et al., 2020, p. 360).

Self-perception. Students' beliefs about themselves fluctuate through the transition in a variety of ways. The identities adolescents create are heavily influenced by the external structures and social interaction they encounter (Eccles \& Roeser, 2011) and become an avenue of concern during this age (Wentzel et al., 2019). When disruptions interfere with those outside forces, they can carry heavy consequences in a student's emotional development and belief in one's self (Eccles \& Roeser, 2011). Self-perception and self-esteem have been shown to be associated with the educational disruption caused by intraschool transitions (Alspaugh, 2001). Events that forced students to evaluate their own dispositions, even if they were caused by outside forces, were associated with dropping out of high school (Dupéré et al., 2018). During the transition to high school, at a heightened time of newly acquired autonomy, students reflect on themselves due to the many social and academic changes they are vulnerable to (Wentzel et al., 2019). They question even the most specific aspects about themselves as they initially navigate high school, for example, their ability to protect their own physical safety (Wentzel, et al., 2019). However, no facet of their self-perception is scrutinized more than their academic ability and competence.

The transition to high school is a time-specific influence on students' academic selfperception and can force them to reevaluate their abilities in mediating other realms of their lives such as with social and self-regulatory issues (Wentzel et al., 2019). Due to this, the selfefficacy of a student can deeply determine how well they overcome the universal deterrents imposed on them when entering high school. Self-efficacy in students emphasizes potential 
performance ability rather than personal qualities such as reflections of self-esteem or selfconcept, which directly effects academic aspects such as motivation, engagement, and performance (Anderson et al., 2019). In fact, desiring to feel academically competent is a crucial element of motivation all the way through high school, not just upon entry (Wentzel et al., 2019). However, there exists a decline in self-perceptions of competency as students progress from elementary through high school, with the greatest decline in grades surrounding the transition from feeder schools, then followed by a plateau after the ninth grade (Davis et al., 2014). Silverthorn, Dubois, and Crombie (2005) found that achievement in one year was a greater predictor of self-perception of academic ability the next in grades eight through ten more than any other levels. This supports findings that efficacy beliefs are more impactful on the academic and behavioral outcomes of adolescents than those of younger students (Davis-Kean et al., 2008) Mixed research on self-perception through the transition suggests this is not always negative. Attitudes toward academics do not always suffer the same as academic behaviors during disruptions (Benner, 2011). For example, certain studies have shown a decrease in academic concerns from the eight to the ninth grade (Benner \& Graham, 2007; Smith et al., 2008). Others have shown that self-efficacy remains unchanged or even grew for students from middle to high school (Benner, 2011). During the ninth-grade, self-efficacy parallels academic performance patterns caused by the transition, as it declines during the middle of the year but recovers toward the end and improves going into the tenth grade for students successfully acclimating to high school (Benner, 2011; Davis et al., 2014). When students exhibit positive self-belief, they can also build resiliency to ameliorate against the disengagement many of them resort to when faced with difficulties they seemingly cannot overcome (Anderson et al., 2019; Rosen et al., 2019). Overall, when students in mid-level feeder schools and high school foster 
positive self-perceptions, particularly academic efficacy, they envision better outlooks toward their future prospects along with greater motivation to reach their potential (Dedmond, et al., 2006). Students finding value in school has been shown to improve attendance, engagement, achievement (Anderson et al., 2019). If students cannot adjust to the transition and continue to feel isolated, stressed, or incompetent, their self-perception deteriorates, which will lead to the external behaviors linked to dropping out (Mizelle, 2005). It is imperative that mid-level feeder school and high school teachers strive to promote positive self-perceptions in as many students as they can.

\section{Relational Consequences}

Transitional factors that cause stress to students can result in psychological consequences. Both transitional factors and psychological consequences can lead to relational issues as well for students. Relational consequences refer to how students connect to their surroundings as well as how engaged they are in their academics.

Connectedness and engagement. It has been well documented that academics decline significantly across the transition to high school (Benner et al., 2017). The opposite can be said for feeling lonely, isolated, and not belonging, as these significantly increase for students as they enter high school (Chapman \& Sawyer, 2001; Cooper \& Liou, 2007; Gentle-Genitty, 2009) and are also linked to depression (Benner et al., 2017; Newman et al., 2007). A student's socioemotional capital can be enhanced or damaged due to their level of school connectedness (Benner et al., 2017). Uvaas and McKevitt (2013) defined school connectedness as the combination of the level of belonging students feel with their school along with the quality of their peer and teacher relationships within that setting. Newman et al. (2007) illustrated its importance, stating, "Disruption of this sense of belonging or connectedness has implications for 
mental health, physical health, and academic achievement" (p. 18). Essentially, as students' stressors increase, so does the disconnect from their school (Roybal et al., 2014), which can have catastrophic results. The relationship between connectedness and the disruptiveness of the transition to high school is cylindrical in nature as each can cause the other. Students who feel more disconnected from their school experience a more difficult transition, while increased instability of the disruption for students can cause them to be more disconnected from school (Benner et al., 2017). It is common for students to feel a lack of connection to an unfamiliar environment (Newman, 2007; Roybal et al., 2014), especially if they have trouble adapting. Students experience significantly lower levels of connectedness and involvement in high school than in mid-level feeder schools (Uvaas \& McKevitt, 2013), with their greatest concern of belonging being making friends in a new environment (Wentzel et al., 2019). Adolescents have an innate need to belong and be socially accepted by peers (Roybal et al., 2014). Those who use the change to forge new social pathways and elevate their social status through school improve their socioemotional capabilities, which eases the transition (Benner, 2011). Newman et al. (2001) stated, "connectedness to family, peers, and school is viewed as a mesosystem that helps buffer the challenges of the high school transition and reduces the likelihood of depression and negative or risky health behaviors" (p.445). They exhibit less drug and alcohol abuse, sexual promiscuity, and absenteeism (Uvaas \& McKevitt, 2013). While connectedness can lead to social acceptance (Roybal et al., 2014), the benefits also spill over to academics.

School connectedness has a significant association to increased motivation and academic success (Roybal, et al., 2014; Uvaas \& McKevitt, 2013). Students who feel a sense of belonging to a school find it meaningful at all levels, while students experiencing exclusion reduce its value (Roybal et al., 2014). One way for teachers and staff to increase students' connectedness to 
school is through innovative, meaningful, and timely intervention in and beyond academics (Newman et al., 2007). Academically, when students identify with their teachers' practices and efforts, along with how a teacher responds to their struggles and worries, builds connection through trust (Brake, 2019). School intervention is necessary for students who lack trust in their teachers because that disconnect puts them at greater risk to dropout (Thayer et al., 2018; Uvaas \& McKevitt, 2013).

When schools show they care by being responsive to the students' basic and developmental needs, both intrinsic motivation and school connectedness grows in students (Eccles \& Roeser, 2011; Ellerbock \& Kiefer, 2013). During the transition to high school, this care is especially shown by dedicated assistance to help students cope with the unique challenges they are facing (Cauley \& Jovanovich, 2006; Newman et al, 2006). For example, Vera et al. (2016) found that attending a high school summer program between the eighth and ninth grade increased connection with personnel and to the environment and resulted in slightly elevated initial academic achievement to their freshman counterparts. No matter the means, intentional programs and activities that raise student belonging in a new setting can have extensive benefits that minimize the disruptiveness of the transition (Ganeson \& Ehrich, 2009).

Intervention does not have to be exclusive to the classroom to be beneficial for incoming freshman. Involvement in extra-curricular activities help new students become integrated in their school (Langenkamp, 2009, Parrish et al., 2011). Students entering high school underestimate the opportunities that come in the form of social clubs and activities they can participate in and the positive impact it can have on their intellect and academics (Smith et al., 2008). Even if they are not interested in school, being part of a community or system bigger than themselves that extra-curriculars provide, motivates them to achieve a certain level of success in 
the classroom (Parrish et al., 2011). When students perceive that schools want them to be involved and will help facilitate their participation, the likelihood they will succeed through high school increases, often at levels higher than they imagined (Cavendish, 2013).

Overall, student perceptions of school climate vary among students because of the high school transition. Increased disparity in connectedness across institutions has proven to be detrimental to students' development; and therefore, mid-level feeder schools and high schools should create consistent feelings for connection and belonging in both arenas (Benner, 2011).

\section{Behavioral Consequences}

Another form of consequence from the transition to high school consists of external actions that are usually reactions from psychological and relational consequences suffered through the disruption. These are measurable behaviors that often contribute and even predict if students graduate high school. The following conveys how the freshman transition relates to absenteeism and discipline issues in high school.

Absenteeism. It is well documented that attendance issues are significantly linked to higher dropout rates in schools (Balfanz et al., 2007; Hazel et al., 2014; Hickman et al., 2008; Lovelace et al., 2017; McKee \& Caldarella, 2016; Mizelle \& Irvin, 2000; Uvaas \& McKevitt, 2013). Students who attend school less than $90 \%$ of the time are four times as likely to dropout of high school (Lovelace et al., 2017). In their study, Allensworth and Easton (2007) found that $87 \%$ of students who missed less than one week of school per year graduated on time in comparison to $63 \%$ for those who missed more than one. Only $22 \%$ of students graduated when their attendance rate dropped below $80 \%$ (Neild \& Balfanz, 2006). However, these numbers are not exclusive to high school as low attendance in younger grades is often an accurate predictor of dropping out of school (Hazel et al., 2014; McKee \& Caldarella, 2016). 
Attendance patterns usually stay consistent from mid-level feeder schools to high school, but because of the disruption caused by the transition, if they do change it is in a negative direction (Benner \& Wang, 2014). A difficult transition often leads to higher absentee rates for freshman than any other level (Cohen \& Smerdon, 2009; Uvaas \& McKevitt, 2013) and contributes to cause freshmen more risk than any other age level of dropping out of school (McCallumore \& Sparapani, 2010). Mid-level feeder schools provide many predictors for ninth grade attendance, but none are stronger than student achievement (Lovelace et al., 2017). Lower mid-level feeder school GPA (Allensworth \& Easton, 2007) and low eighth grade ACT math scores (McKee \& Caldarella, 2016) were associated with increased absenteeism in the ninth grade. Hickman et al. (2008) stated, "The culmination of course performance grades and absenteeism over the academic career of dropouts and graduates seemed to solidify with entry into the ninth grade" (p.12). Roybal et al., (2014) found that attendance grew significantly at the end of eight grade and the beginning of ninth grade but dropped significantly throughout the rest of the year. This drop could be the result of the declining academic performance during the first semester of the freshman year (Hickman et al., 2008; McCallumore \& Sparapani, 2010), and the subsequent stress felt by students after (Roybal et al., 2014). Students performing poorly face fear and embarrassment in a time of a heightened need for social acceptance and in turn resort to disengagement and avoidance (Allensworth \& Easton, 2007).

Mid-level feeder schools are not always effective predictors as a disruptive transition alone can cause decreased attendance. In a study done in Philadelphia Public Schools, about $30 \%$ of dropouts entered high school having no previous failing grades or attendance issues (Neild, 2009). However, they earned so few credits under the new requirements and became disengaged that they attended less than $70 \%$ of the time (Nield, 2009). In urban schools this is 
more common and is the result of a combination of the disparity between students' academic and social abilities at each level and the contextual demands of the larger high schools they attend (Neild et al., 2008).

Freshman absenteeism caused by disengagement due to lack of success illustrates the need for mid-level feeder school teachers to better prepare students for high school and for all teachers across the transition to increase cohesion. High school teachers can also help by being better prepared, as disorganization by schools at the beginning of the year can also increase student absenteeism moving forward (Nield et al., 2008). Some schools have found success in consistent adherence to a district attendance policy and frequent communication of that policy to every child, especially those at risk (Smith et al., 2008).

Discipline. Rarely do attendance and behavioral issues happen in isolation of each other (McKee \& Caldarella, 2016). Like attendance issues, student discipline has widely been associated with higher rates of dropping out of school (Balfanz et al., 2007; Hazel et al., 2014; Hickman et al., 2008; Lovelace et al., 2017; McCallumore \& Sparapani, 2010; McIntosh et al., 2008; McKee \& Caldarella, 2016; Mizelle \& Irvin, 2000; Uvaas \& McKevitt, 2013). Many of the behavioral issues surrounding the transition parallel that of absenteeism. A difficult transition associates with increased behavior problems (Smith, 2006a) and happens at higher frequency than any other age level (McCallumore \& Sparapani, 2010; Smith et al., 2008). These behavior issues crop up in many different forms. One way is that with the greater independence they receive from parents and in the school structure, they tend to skip class more (Neild et al., 2008). A prominent behavioral issue that arises during the transition is cheating (Anderman \& Midgley 2004). Students practice this more after the transition because of their perception of an increased emphasis on performance goals versus the decrease in mastery goals imposed upon 
them by the credit accumulation requirements (Anderman \& Midgley, 2004). When that perception subsided, so did the self-reported cheating levels (Anderman \& Midgley, 2004).

Mid-level feeder school behavior can also be a predictor of student success, even as far as later in high school (Moore et al., 2016). Receiving at least one out-of-school suspension in midlevel feeder school is linked to higher dropout rates (Lovelace et al., 2017). Only 29\% of early mid-level feeder school students with negative behavior marks graduated high school within five years, and only $20 \%$ did with more severe or multiple infractions resulting in out-of-school suspension (Balfanz et al., 2007). A study conducted by McIntosh et al. (2008) found that problem behavior in eighth grade was a statistically significant predictor of academic achievement in ninth grade, and that the reversal, eighth grade academic performance significantly predicting ninth grade behavior, was also true. Furthermore, the percentage of students with challenges in academics but not behavior was four times of those with challenges in behavior who succeeded academically (McIntosh et al., 2008). Essentially, students with disciplinary issues were much more likely to have issues in both areas than those with academic issues and consequently reach a critical likelihood to dropout (McKee \& Caldarella, 2016).

One way to combat disciplinary issues is to have early intentional interventions specific to those students at risk. To implement this, these students need to be identified early enough that they can still benefit from the intervention before they succumb to the dropout path, which is what usually happens when waiting to address these issues until high school (McIntosh et al., 2008). Some high schools have had success with universal positive behavior systems and restorative justice for disciplinary infractions (Hazel et al., 2014). This involved having ninth grade staff on committees dedicated only to at-risk interventions and proactively collaborating plans to assist students in changing behaviors (Hazel et al., 2014). At both the mid-level and 
high school levels, another successful practice has been that teachers teach students how to behave according to their expectations instead of being strictly reactionary to student behaviors, especially since problem behavior directly forecasts academic success (McIntosh et al. 2008).

\section{School Response}

Substantial evidence has shown the many complexities and subsequent consequences facing students entering high school. The turbulence through the transition from mid-level feeder schools to high school results in increased stress, lower academic achievement, and a greater likelihood of dropping out for students unable to overcome it (Neild, 2009). Schools are fully aware of this issue and have gone to extensive lengths to lessen the negative effects for freshman by implementing transition programs and activities (Ganeson \& Ehrich, 2009). Schools see improved attendance, achievement, and retention when their programs are effective (Cauley \& Jovanovich). In fact, the more reform practices that are implemented by schools that specifically target the freshman transition results in greater student learning (Morgan \& Hertzog, 1998; Neild, 2009). When combined, these practices create a multi-faceted program that blend youth development with rigorous, enriched learning opportunities that display relevance to students' future and simultaneously offer personal, academic, and social support (Feller, 2003). In fact, Kennelly and Monrad (2007) found that schools with fully implemented programs have dropout rates one-third the size of those schools without these systems in place. An effective school response is imperative to combat the potential detriments of the transition from mid-level feeder schools to high school.

The following outlines the research on school response to the transition from mid-level feeder schools to high school. The outline starts with the shared accountability required to implement reform, moves into different programs and supports, such as small learning 
communities (SLC), that have been shown to be effective and finally concludes with success stories that have been documented from different school districts interventions.

\section{Shared Accountability}

While freshman transition interventions vary greatly, one way school districts can start to make progress is by understanding the shared accountability that is required to start and sustain them. Accountability appears in every community that contributes to the freshman transition. With that, the following describes the responsibilities of the systems outside of the high school, starting with mid-level feeder schools and moving outwards to more peripheral roles.

Mid-level feeder school intervention. The highest overarching priority of staff at every school level should be to have their students graduate and be prepared for their lives beyond (Nelson et al., 2007). It has been well established that there are multiple disconnects between lower level schools and high schools in easing the transition for students. The essential responsibility in mid-level feeder schools is to take pride in promoting well-prepared students into high school (Nelson et al., 2007). High school teachers agree, suggesting higher rigor and enrichment in earlier grades' curriculum (Akos \& Galassi, 2004). Yet strong evidence shows students come to school unprepared and become off track, and the high school teachers must "catch them up" academically (Neild, 2009). Mid-level feeder school teachers must realize that the struggles of freshmen are an extended responsibility that applies to them and not just to high school teachers (Mizelle, 2005). At middle levels, teachers need to not only respond with challenging curriculum and expectations, but also programs that specifically target preparation for the transition into high school (Ganeson \& Ehrich, 2009; Mizelle, 2005; Parrish et al., 2011).

One area where mid-level feeder schools can improve on the transition is the way they interact with their high school counterparts. Not only is the transitioning students' input 
overlooked in implementing and sustaining a transitional program, often mid-level feeder school and high school teachers are as well. According to Cooney and Bottoms (2002), most high school teachers in their study had never even met with their mid-level feeder school teachers. Feeder schools should create partnerships that focus on alignment of as many academic functions as possible (Parrish et al., 2011).

One responsibility that ties mid-level feeder schools to high school, that also involves contributions from outside of that relationship, is data sharing. Mid-level feeder school teachers need to conduct valid assessments that puts ownership on their students, interprets the results, utilizes the data to inform decisions and guides instruction (Balfanz, 2011; Neild, 2009; Nelson et al., 2007). These results, along with data from attendance rates and behavior monitoring, should also contribute for early identification of potential students at risk (Balfanz, 2011). The resulting information needs to be communicated throughout all levels, so timely and individualized supports can be carried out to keep students on track to succeed (Balfanz et al., 2007, Parrish et al., 2014). The early warning system needs to be teacher friendly and ideally feed into a comprehensive intervention system (Balfanz, 2011; Balfanz et al., 2007). An innovative method school districts are using are called machine learning algorithms, which is technology that uses internal and external data, to illustrate potential educational trajectories for student (Sorenson, 2019). Using this method can be costly and technologically demanding, but its capabilities of making accurate predictions using more comprehensive data and communicating it more efficiently may make it worth it (Sorenson, 2019). Regardless of the means, these data need to be communicated better throughout the age levels. Without proper teacher coordination, early indicators are underutilized or even completely ignored across the transition (Balfanz et al., 2007). 
Peripheral staff responsibilities. The responsibility of creating an optimal transition to high school for students does not solely fall on the classroom teachers (Nelson et al., 2007). Administrators, coaches, counselors, and community members must all contribute to ensure a smooth transition (Morgan \& Hertzog, 2001; Nelson et al., 2007). Therefore, peripheral staff in this case refers to all individuals considered stakeholders in the school response to the freshman transition that are not classroom teachers. Leaders at every level must strive for universal buy-in from all these entities (Nelson et al., 2007, Smith, 2007).

Peripheral staff must continuously work with mid-level feeder school and high school teachers to overcome challenges in implementing and sustaining an effective transition program. Parrish et al. (2011) outlined these challenges as (a) generating and properly managing the fiscal investment necessary to carry out the programs, (b) creating opportunities for vertical teaming and collaboration of staff in multiple locations, (c) mediating conflict of "blame" conveyed by teachers on others, (d) identifying and tracking students between middle and high school grades and the severity of each of their situations, (e) creating a new transparent culture of effective academic climate and high standards with staff and students, and (f) communicating externally the academic success, or lack thereof, to the right audiences. At every stage of the program implementation, peripheral staff, especially administrators, can carry out the suggested responsibilities to increase the chances of the program' s success.

Evaluation of a program is an essential component that should be continuously performed at every stage of the program's development (Morgan \& Hertzog, 2001). Stakeholders first need to be selected and, based off needs and possible gap analysis using comparative research of high performing schools' transitional practices, decide on a program to implement (Mathis, 2004). A criterion of success used for evaluation must then be developed for which the program 
should be measured by participation of students and parents in activities and programs, retention rates, standardized test scores, credit accumulation, and graduation rates (Morgan \& Hertzog, 2001). Data sharing processes need to be established and utilized to create cohort specific programs to account for the changes in students and contexts such as changes in state and district policy (Morgan \& Hertzog, 2001; Parrish et al., 2011).

Administrators may have to make structural changes, such as rearranging teachers and schedules based on their varying abilities to teach, connect, and motivate students (Hazel et al., 2014) or changing the current layout of their building to benefit freshman. At a district level, certain schools have found success when they streamlined their district feeder patterns because cost was reduced, staff coordination became easier, students were more familiar with their peers, and the overall disruption was eased (Parrish et al., 2011). Once a foundation is put in place, curriculum can be enhanced with the recruitment of business and community members to create connections of relevance between student learning and the outside world for students (Dedmond et al., 2006). As schools and districts find evidence of success of the program and its elements, they should institutionalize them with policies to make them universally known (Nelson et al., 2007). It takes contributions from every resource and constant evaluation, communication, and accountability to ensure the success of a freshman transition program.

\section{Small Learning Communities and the Freshman Academy}

Many high schools have countered the disruption of the transition to high school with extensive structural and philosophical reform. Many do this by installing small learning communities known as freshmen or ninth grade academies (Balfanz, 2011; Bornsheuer et al., 2018; Cauley \& Jovanovich, 2006; Corcoran \& Silander, 2009; Dedmond et al., 2006). These academies are usually in an isolated geographical location within the building specifically only 
for ninth grade classes (Butts \& Cruziero, 2005; Kennelly \& Monrad, 2007; Smith, 2007). By separating freshman, students have a place of comfort to mature and adjust to the rigors of high school (Cauley \& Jovanovich, 2006; McCallumore \& Sparapani, 2010; Reents, 2002). They can adjust to the processes of high school in a more intimate, structured setting familiar to that of mid-level feeder schools (McCallumore \& Sparapani, 2010; Smith, 2007). The closed-off area also reduces the distraction and intimidation that can be felt from interaction with upper classmen and instead uses the first year to slowly integrate them in with their older peers (Kennelly \& Monrad, 2007; Smith, 2007). Under this structure, the freshman class is easier to manage and monitor by teaching and administrative staff (McCallumore \& Sparapani, 2010).

Another way the intimate setting of the small learning community format benefits both students and staff is that it produces stronger relationships between them (Cauley \& Jovanovich, 2006). Students receive more personalized and individualized attention from teachers unified under the freshman philosophy (Cauley \& Jovanovich, 2006; Kassissieh \& Oxley, 2008; McCallumore \& Sparapani, 2010; Reents, 2002), and is more specific when that freshman staff is broken down further into teams, interacting with its own group of students (Smith, 2007). Students enter high school have differentiating degrees of skill level and risk profiles that can be easily misrepresented in the data high school staff receive (Hazel et al., 2014). The organization of cross-disciplinary student-oriented support teams within the freshman academies allow teachers to collaborate to offer differentiated interventions specific to most every student at risk (Hazel et al., 2104). For example, teachers can coordinate the amount of work assigned by each of them in order to not overload and overwhelm students as they adjust to the transition (Ganeson \& Ehrich, 2009), or offer a "Zero Hour" built in to their schedules where students can receive additional academic and personal attention (Hazel et al., 2014). The coordinated effort 
increases the likelihood students feel engaged and connected to school which has been shown to be a predictor of student success (Davis et al., 2014). In fact, Davis et al. (2014) defied most literature when their study revealed that interdisciplinary teaming in SLC's closed the relationship quality gap and increased engagement of at-risk students across the transition.

While important, stronger student-teacher relationships created by the freshman academies are not enough to ensure success (Butts \& Cruziero, 2005; Kassissieh \& Oxley, 2008). Teachers need to collaborate on academics with a focus on research-based quality instruction and creating stronger communication and relationships with family members (Balfanz, 2011; Kassissieh \& Oxley, 2008). Peripheral staff can help by increasing resource allocations to the freshman academy and creating common planning time for departmental and interdisciplinary collaboration and professional learning (Hazel et al., 2014; Kassissieh \& Oxley, 2008). The returns have been promising when freshman academies are prioritized universally in a district. In comparison to pre-academy data, McIntosh and White (2006) found that ninth grade SLC's had produced improvements in parent relations, staff and student morale, attendance rates, and behavior. Most importantly, there was an increase in classroom academic success and achievement on standardized test scores (Kassissieh \& Oxley, 2008; McCallumore \& Sparapani, 2010). The benefits increase when additional supports are also implemented.

\section{Supplemental Freshman Transition Supports}

Schools see a rise in student achievement success the more high quality supports they offer all students transitioning into high school (Butts \& Cruziero, 2005; Kennelly \& Monrad, 2007; Mac Iver, Epstein, Sheldon, \& Fonseca, 2015; Letrello \& Miles, 2003; Neild, 2009), not just those perceived to be at risk (Johnson et al., 2014). Many schools are turning to structural reform such as creating freshman small learning communities (Balfanz, 2011; Bornsheuer et al., 
2011; Cauley \& Jovanovich, 2006; Corcoran \& Silander, 2009; Dedmond et al., 2006) and academic reform that uses extensive research-based collaborative professional development to increase collective efficacy and improve instruction (Balfanz, 2011; Butts \& Cruziero, 2005; Lee, Zhang, \& Yin, 2011). However, schools have also integrated a variety of additional supports to supplement their academic and structural reforms that cater to freshmen. Mac Iver et al. (2015) found that $89 \%$ of schools and $70 \%$ of students in their study reported having at least one school sponsored event to help them prepare. These efforts happen throughout both the transition and the freshman year (Morgan \& Hertzog, 2001).

One way to help students is to ensure multiple ways for additional academic supports are accessible for students throughout the transition (Parrish et al., 2011). Schools have implemented summer programs specific to incoming ninth graders (Hazel et al., 2014; Horwitz \& Snipes, 2008; Kennelly \& Monrad, 2007; McCallumore \& Sparapani, 2010; Parrish et al., 2011; Smith, et al., 2008; Vera et al., 2016). Ideally, these courses are taught at the incoming high school setting with high school staff so students can create connections and familiarity with both (Horwitz \& Snopes, 2008). Struggling students who attended summer school before high school had higher attendance rates, fewer behavior issues, higher GPA, and fewer course failures than their counterparts who chose not to participate (Fulk, 2003). Even students who attended a weeklong summer class focused on high school orientation saw increased success the following year (Hazel et al., 2014). Vera et al. (2016) conducted a study using a pretest and posttest measure of different academic attributes found to contribute to success for students. They found students who participated in a summer program had an improved sense of belonging to the school and staff and improved self-efficacy. These students also became better informed about what it takes to be successful in high school and made a deeper connection between school 
success and future career plans, which resulted in them valuing education more and showing increased motivation to succeed (Vera et al., 2016).

In addition to summer school, school districts have offered additional academic supports and remediation in many ways. Many schools require students to take a preparatory Freshman Seminar course their first semester in high school (Dedmond et al., 2006; Kennelly \& Monrad, 2007; Parrish et al., 2011; Smith, 2007; Smith, et al., 2008), which primarily consist of two objectives. The first goal is to improve study skills, time management, and deal with the academic rigors of high school (Smith, 2007). The second objective of the Freshman Seminar course is to illustrate the impact schoolwork has on life following graduation (Dedmond et al., 2006). They achieve this with support of local higher education institutions, and community and business partners, as they enlighten students to the possible directions for their future (Dedmond et al., 2006). When freshman experience meaningful context toward career development in the curriculum, their self-esteem and motivation levels increase (Dedmond et al., 2006).

Some schools offer extra intentional supports for struggling students as they progress through their freshman year, specifically in math and English (Smith, 2007). Systematically, schools change the way they promote freshmen by double-dosing math, English, and remediation courses for struggling students their first year (Parrish, et al., 2011; Smith, 2007). They accomplish this by switching from year-long courses to block scheduling to achieve more credits (Kennelly \& Monrad, 2007), decreasing homeroom size (Fulk, 2003), eliminating the ability for freshmen to drop classes, and even offering courses outside of school to remain on track for credit accumulation (Butts \& Cruziero, 2005). Schools also offer tutoring programs both in and outside of school from staff and older students (Kennelly \& Monrad, 2007; Parrish et al., 2011; Smith, et al., 2008). The tutoring is often built into other student-to-student mentoring programs 
that not only provide academic support, but relational and contextual support from a nonauthoritative upperclassman (Balfanz, 2011; Fulk, 2003; Letrello \& Miles, 2003; Mizelle, 2005).

Mid-level feeder schools and high schools also collaborate on transitional events and programs that help both students and families (Letrello \& Miles, 2003; Mizelle, 2005), although less than half of schools self-report succeeding in this task (Mac Iver et al., 2015). Schools should involve parents during their students' eighth grade year to help them prepare for high school and educate them on upcoming curriculum and expectations (Dorman, 2012; Hazel et al., 2014; Mizelle, 2005; Oakes \& Waite, 2009).

Mid-level feeder schools and high schools can coordinate different forms of freshman orientations (Dorman, 2012; Fulk, 2013; Hazel et al., 2014; Morgan \& Hertzog, 2001; Oakes \& Waite, 2009). These can consist of meet-the-teacher nights, campus tours, student shadowing, high school student question and answer panels, and informational meetings with counselors (Dorman, 2012; Hazel et al., 2014; Letrello \& Miles, 2003; Mizelle, 2005; Oakes \& Waite, 2009). The main premises of these supports should be to alleviate anxiety and fears of incoming freshmen and their parents and debunk myths they may perceive (Dorman, 2012; Letrello \& Miles, 2003; Morgan \& Hertzog, 2001). All these supports should be started in mid-level feeder schools and carried throughout entire freshman year or further (Morgan \& Hertzog, 2001). High schools report they were excelling in communicating these expectations once students enter high school but needed to improve in keeping parents engaged in their students' progress and participation throughout the rest of the freshman year (Mac Iver et al., 2015).

\section{Successful Freshmen Transition Programs}

Extensive research shows the different school responses that contribute to the improvement of the freshman transition for students. In Philadelphia, less than $50 \%$ of their 
students had a chance to graduate within four years, mainly due to absenteeism (Useem, Nei, \& Morrison, 2001). Within two years of implementing the freshman academy model, their overall attendance rate grew by more than $15 \%$ (Useem et al., 2001). Scott County in Kentucky was forced to make changes after $45 \%$ of their incoming freshman were likely to fail one or more classes (Smith et al., 2008). They implemented a ninth grade "school-within-a-school" and their freshman promotion rate grew by $11 \%$, standardized test scores increased to six points above the state average, and disciplinary issues heavily declined (Smith et al., 2008).

Driven by their low math scores, Muhlenberg South High School in Kentucky followed that same model and saw those scores increase by $19 \%$ in just one year (Smith et al., 2008). A Mid-Atlantic Urban High School implemented a peer group connection program and saw that atrisk students who participated had a $13 \%$ higher graduation rate then their at-risk peers who did not (Johnson et al., 2014). One high school in Georgia found improvement in student achievement simply by requiring a "Freshman 101" class that taught essential high school skills, including but not limited to time management, study and test-taking skills, career alignment, and social tolerance (McCallumore \& Sparapani, 2010). Halifax County Schools in Virginia found their success by implementing a comprehensive career connected transition program for all levels supported by surrounding businesses and higher education institutions (Dedmond, 2006). They enhanced this program with a comprehensive array of supplemental supports as students entered high school.

Programs in mid-level feeder schools have also shown to benefit performance of freshmen. George Mason University has partnered with many school districts to create an early identification system of at-risk students in middle school and then provided interventions conducted by college students that included summer academies, family workshops, and tutoring 
all (Dedmond et al., 2006). Smith et al. (2008) found schools in Georgia and Mississippi that implemented rigorous, team-based interdisciplinary curriculum and collaboration in seventh and eighth grades. These resulted in higher test scores, better course passing rates, and decreased behavioral problems, and for the Georgia school, commendation from the state (Smith et al., 2008). Another program being run throughout the country is the Diplomas Now that spans from mid-level feeder schools to high school (Balfanz, 2011). It has shown to increase student success using whole-school reform, early warning systems, peer mentoring and team-based work for those districts that executed it (Balfanz, 2011). It is evident that school districts should not leave the freshmen transition unaddressed and should find the system of supports that best suits their students' needs in their response.

\section{Vertical Collaboration}

Unique internal and external forces coalesce to pose challenges specific to every student entering high school. While schools have made numerous efforts to quell the disruptiveness the transition to high school causes, there exists an academic disconnect vertically feeder and high school teachers. Substantial literature exists on collaboration throughout a school district. The following outlines the general characteristics and focuses of collaboration, and the increase in collective efficacy in the staff as a result. The outline continues with an examination of the current research on vertical collaboration and teaming between high school and feeder school teachers and the obstacles they face in collaboration. This section will conclude by illustrating the effect educator collaboration has on student achievement.

\section{Professional Learning Communities and Teacher Collaboration.}

Quality teacher collaboration has increasingly been shown to have substantial positive effects on school improvement through teachers taking shared accountability toward continuous 
improvement (Ronfeldt et al., 2015). Johnston and Tsai' s (2018) definition of collaboration incorporates Newmann et al.' s (2001) key elements of IPC. It states that teacher collaboration is an interaction between colleagues focused on improving and enhancing instruction, curriculum, assessment and supports for students (Johnson \& Tsai, 2018). Teachers often rely on each other to assist in their professional development before any other resource. Berry et al. (2009) found teachers turn to peers for initial support $68 \%$ percent of the time. This is often informal as teachers seek out peers they are close with, but also those who they feel are experts in the area where they need assistance regardless of grade level (Stevenson, 2004).

Knowing this, schools can capitalize on that interaction by formalizing and structuring teacher collaboration to make it more intentional and utilizing the variety of staff expertise at their disposal (Şahin \& Cemaloğlu, 2015). A collaborative culture makes schools more attractive to teachers and tends to result in lower staff attrition rates (Berry et al., 2009). A strong collective collaborative culture has also been linked to higher student achievement (Gruenert, 2005; Lee \& Louis, 2019), regardless of the varying levels of collaborative efforts from each teacher (Ronfeldt et al., 2015). In fact, collaboration has shown to be more effective to turning around low performance in schools than removing and replacing ineffective teachers (Berry et al., 2008). This has some schools in constant pursuit of the strongest collaborative culture they can achieve.

Many factors need to be considered to create a strong collaborative culture. The most prevalent factor in research is to allot proper time for PLC's to interact (Balfanz, 2011; Berry et al., 2008; Dorman, 2012; Gajda \& Koliba, 2008; Goddard, Goddard, Sook Kim, \& Miller, 2015; Goddard \& Kim, 2018; Kallemeyn, 2014; McIntosh \& White, 2006; Slick, 2002). Many schools take it a step further and embed a common planning time into their schedule throughout the year 
(Hazel et al, 2014; Jacobson, 2010; Kallemeyn, 2014; Parrish et al., 2011; Şahin \& Cemaloğlu, 2015). Teachers report that when given ample opportunity to interact with their colleagues, they are more apt to actively participate (Johnston \& Tsai, 2018). This means that the duration of each session needs to be long enough and collaboration should also be sustained continuously throughout the school year (Akiba, 2012; Goddard et al., 2015; Goddard \& Kim, 2018; Kallemeyn, 2014; McIntosh \& White, 2006; Nelson et al., 2007). The demands teachers face make efficiently structuring that collaboration crucial for maximizing their depleted availability.

To create high quality teacher collaboration, creating space and time is simply not enough (Gajda \& Koliba, 2008). Before schools can grasp a better understanding of how to structure $\mathrm{PLC}^{\prime} \mathrm{s}$, they must first be intentional with the purpose and objectives of the collaboration. Through this, the entire staff can work under a recommended shared vision (Nelson et al., 2007; Parrish et al., 2011; Roybal et al., 2014). School leaders should implement a strategic PLC plan and structure that aligns with this mission (Akiba, 2012; McIntosh \& White, 2006). When schools have common frameworks that guide their collaboration strategies and goals, they have higher quality experiences than those who collaborate without a framework or fail to collaborate at all (Gajda \& Koliba, 2008; Youngs, Holdgreve-Resendez, \& Qian, 2011). Staff allocation is a key contributor to PLC's as it is not about quantity of staff but the quality of their collaboration (Nelson et al., 2007). This can be critical to a school's IPC as well. Jacobson (2010) recommended schools build IPC with PLC's through coordinating the teams and their responsibilities in a manner that builds upon previous progress and does not haphazardly unfold or counteract others' work. This will produce more thorough follow through on initiatives.

On top of making the proper structural decisions, school officials play a pivotal role in implementing and sustaining effective $\mathrm{PLC}^{\prime} \mathrm{s}$ by fostering a trusting atmosphere uniting their 
staff under their commitment to students (Berry et al., 2008; Gruenert, 2005; Lee et al., 2011).

These leaders can shift policy to build and guide stronger communities of practice that is critical for sustainable school improvement (Corcoran \& Silander, 2009; Lee \& Louis, 2019). In fact, the degree to which principals buy-in to $\mathrm{PLC}^{\prime} \mathrm{s}$ is a significant predictor of teacher collaboration which has a significant relationship with teaching quality, making collaboration a partial mediator between strategic leadership, and teaching quality (Goddard et al., 2015; Ismail et al., 2018). In other words, principals' instructional leadership and teacher collaboration are indirect predictors in different levels of student achievements among schools (Goddard et al., 2015). School leaders try to mold their schools into learning organizations using PLC's (Lee \& Louis, 2019). PLC's strive to practice strategic renewal, as they are on a continuous improvement cycle using processes of inquiry that both enhance what is currently working for teachers while simultaneously looking for innovative change (Gajda \& Koliba, 2008; Kallemeyn, 2014, Levine \& Marcus, 2007). Strategic renewal can be achieved by leaders breaking away from bureaucratic norms that enforce particular types of instruction and instead foster democratic networks (Şahin \& Cemaloğlu, 2015, Slick 2002) that are responsive to the daily pressures of teaching as they pursue progress toward larger ideals (Lieberman, 2000). Ohlsson (2013) stated the importance of reflection in this process:

Teachers' team learning is an ongoing process of interrelated activities including a strong team orientation and an interdependently creation of joint tasks and problem solving; a positive challenging team atmosphere that facilitates the emergence of collective reflection loops necessary for double-loop team learning, increased collective competence and professional growth. (p. 308) 
Teachers who use the knowledge gained from the reflective networks often recreate meaning of scholarship and examine their own practices and ideals as a result (Lieberman, 2000).

For this reflection, networked PLC's should disseminate newly obtained knowledge and skills throughout the school for practice through exchange in collegial workgroups of materials and data (Prenger, Poortman, \& Handelzaltz, 2019). Schools should also use the results from its knowledge application to guide further learning when they reconvene (Prenger et al., 2019). Therefore, principals and other PLC facilitators should engender the proper atmosphere consisting of transparency, positivity, trust, and respect where teachers feel safe and motivated to participate and contribute (Lee \& Louis, 2019; Şahin \& Cemaloğlu, 2015). Teachers will also be more apt to exert more effort toward the PLC's goals if they feel cared about and supported by the principals and other school leaders through their transparency, follow-through, and utilizing teachers' contributions (Louis \& Murphy, 2017, Nelson et al., 2007). Slick (2002) agreed and recommended providing opportunities for teachers to gain a sense of self-worth and feeling of success by providing reinvigorating social and professional activities.

Schools and districts can take concrete measures to produce these proper learning environments for teachers. This can be accomplished through implementing protocols to guide and scaffold collaboration and produce relevant and continuous follow through (Johnston \& Tsai, 2018). When leaders provide guided support, over twice as many of the agreed upon applications are carried out in class (Levine \& Marcus, 2010). A leader's role in enforcing these protocols is a delicate process that can both enhance and constrain teacher improvement (Levine \& Marcus). Without proper structure, teams may not make good use of their time and often veer into different directions (Jacobson, 2010). This may push teachers to not contribute or offer glimpses into their own practice, which can provide key insight to promote learning and capacity 
building (Levine \& Marcus, 2010). Too much structure may diminish teacher enthusiasm and make them feel less valuable (Jacobson, 2010). PLC facilitators need to find a healthy balance between top-down and bottom-up protocols, open-ended and structured processes, and inquiry oriented and restorative driven improvements (Jacobson, 2010). In other words, school leaders can benefit greatly by providing teachers the foci of inquiry, measures of progress, avenues of communication, and latitude for data driven decision making, while intervening only to reengage or refocus teams falling off track of the main objectives (Levine \& Marcus, 2010).

Key characteristics of collaborative protocols are routine meetings in systems of teams, group norms of challenge and respect, and discussion facilitation (Kallemeyn, 2014). High functioning teacher teams engage in honest dialogue based on experience, student-data, and school need to create a problem-solving atmosphere (Gajda \& Koliba, 2008), which can make team members feel a sense of belonging and that they are a vital component to the success of the school (Slick, 2002). This illustrates the importance for leaders to use large amounts of both student data and evidence of effective practices to evaluate and guide further collaboration (Corcoran \& Silander, 2009; Kallemeyn, 2014, Nelson et al., 2007). Establishing a set of criteria to measure data against and evaluate collaboration with promotes a results-oriented culture (McTighe \& Emberger, 2006, Nelson et al., 2007). The use of statistical data also provides more freedom for peers to critique and help each other more effectively and respectfully (Berry et al., 2009). Structural implications that promote proper data usage and analysis are (a) to ensure accessibility to data (Campbell \& Levin, 2009); (b) to create a universal academic language around data (Parrish et al., 2011); and (c) to provide proper avenues to communicate using technology such as discussion boards (Nicholson \& Bond, 2003). 
Leadership, structure, and culture all play important roles in the success of teacher collaboration and $\mathrm{PLC}^{\prime} \mathrm{s}$ and require great effort from everyone involved. However, achieving collective goals of student success and professional improvement are benefits of an ongoing reflective, capacity building collaboration (Egodawatte et al., 2011), making it a worthwhile investment for school districts to undertake.

\section{Collaboration Focus and Instructional Program Coherence}

The focus of collaboration is as important as having an optimal environment for it to occur (Means, 2010). While professional development has historically been expected to center around content, it has since deviated to focus on process, practice, and climate (Nelson et al., 2007). The overwhelming majority of collaboration objectives can be classified within one or a combination of the domains of IPC: curriculum, instruction, assessment, and academic climate (Newmann et al., 2001). The following examines the literature on the focus of teacher collaboration, especially regarding IPC.

Quality collaboration occurs both vertically and horizontally, between classrooms, teaching teams, and buildings so teachers are on the same page with academic, social, and organizational issues (Berry et al., 2008; McCallumore \& Sparaparni, 2010). Collaboration identifies and often reaffirms the collective academic expectations set forth by the department, school or district (Akos \& Glasssi, 2004). Driven by the universal goal of increasing student achievement, these expectations are usually data driven and often define and communicate rigor to staff throughout the organization (Cooney \& Bottoms, 2003; Gilmer, 2010). Collaboration activities of successful schools bring administrators, counselors, and teachers at all levels together to learn about different courses, curriculum, requirements, instructional practices, and programs of their peers and respected schools (Mizelle \& Irvin, 2000). This cooperative work 
often aligns the goals and key sets of beliefs that underlie educators' teaching pedagogies (Eccles \& Roeser, 2011).

A fundamental focus of collaboration is on the vertical alignment of curriculum and standards with focus on student success (Cooney \& Bottoms, 2003; Gilmer, 2010; Ronfeldt et al., 2015; Parrish et al., 2011; Schlosser, 2015; 2015; Sekulich, 2019). Curriculum mapping should be practiced through ongoing communication between grade levels and school buildings (Kowal, 2002; Morgan \& Hertzog, 2001; Sekulich, 2019). Mapping gives teachers a better breadth of understanding of the entire learning pathway of students from kindergarten through high school and better define their place within it (Gilmer, 2010; Kowal, 2002). Being able to clarify what students need to know going into and upon completion of grade levels allows teachers to avoid and rectify gaps in the scope and sequence of learning (Crawford, 2011; Parrish et al., 2011, Smith, 2007). With awareness of what is being taught in other grade levels, teachers can maximize instructional time as to not overlap taught curriculum but rather build upon students' previously acquired knowledge and spend the appropriate time on specific content. They can also make better informed recommendations for students as they are promoted (Kowal, 2002; Parrish et al., 2011). Another benefit of vertical teaming is that it creates better collegiality by developing added respect of others' responsibilities in the learning progression (Crawford, 2011). Unfortunately, curriculum alignment is common within schools, but more difficult to achieve across buildings due to logistic and funding challenges (Parrish et al. 2011).

Another major focus of collaboration is improving instructional practice and alignment to curriculum (Schlosser, 2015; Smith, 2006a). Instructional collaboration is found by teachers to be more extensive and helpful than other areas of focus (Ronfeldt et al., 2015). Johnston and Tsai (2018) found that when the volume of feedback teachers received from their peers 
increased, so did their positive perception that the feedback was helpful in improving their instruction. Teacher collaboration has been proven to improve teaching quality (Ismail et al., 2018) as teachers become less isolated in their instructional methodology (Gilmer, 2010). Strong department and vertical teams increase opportunities for dissemination of practices throughout the teaching staff (Akiba, 2012; Corcoran \& Silander, 2009). These high functioning teams use collegial feedback to make purposeful adjustments to improve the quality and merit of their practice to increase student learning (Donohoo \& Katz, 2019; Gajda \& Koliba, 2008). Educators do this by working together to experiment through the employment of multiple student-centered approaches. They then follow the evidence to continue those strategies found to be effective (Corcoran \& Silander, 2009; Roybal et al., 2014) and also align with required state and local curriculum frameworks (Schlosser, 2015, Nelson et al., 2007).

It has been shown that focusing collaboration on instruction is not only helpful to teachers, but it also builds their instructional capacity as well as that of the overall staff (Crawford, 2011). These teachers tend to keep students more engaged with interesting classroom strategies (Butts \& Cruziero, 2005). Teachers engaged in more collaboration were more likely to employ differentiated instruction and pedagogy that was more challenging and thoughtful (Goddard \& Kim, 2018). These collaborative efforts result in a deeper understanding of pedagogical practices but also content knowledge within and outside of their grade levels (Birkhead et al., 2017). Birkhead et al. (2017) found that approximately $93 \%$ of their participants improved their math content knowledge by working with peers from other grade levels. In addition to the improvement in teaching quality, teachers also benefited from the personal connections and expertise gained throughout the district (Gilmer, 2010). Overall, teachers "can increase instructional capacity by developing strategies, sharing strategies, and 
discussing the pros and cons of implementation of the various strategies at different grade levels, all for the purpose of improving student learning" (p. 4, Crawford, 2011).

Schools must continually assess their school improvement and provide tangible evidence of its effectiveness (Donahoo \& Katz, 2019; Gajda \& Koliba, 2008). Collecting comparative evidence requires the methods of measurement to be consistent by utilizing student data through common assessments (Jacobson, 2010). Some schools use standardized state tests as a guide to create common assessments (Nelson et al., 2007) because it provides a common measurement system, academic language, and outcomes that can be measured against other schools (Parrish et al., 2011). In fact, collaboration around creating common assessments is often more predictive of school and teacher performance and student achievement than any other collaborative focuses (Ronfeldt et al., 2015). Therefore, teacher teams should collaboratively design, and peer review assessments based on desired learning results and use student work from these measures to identify student misconceptions (McTighe \& Emberger, 2006; Ronfeldt et al., 2015; Schlosser, 2015). The data provide feedback for staff to identify struggling students and intervene to get them on track with their learning (Gilmer, 2010; Nelson et al., 2007).

The data produced from student assessment not only allow teachers to monitor student progress, but also the effectiveness of their own teaching. For this to occur, teachers cannot feel constrained by administration when it comes to discussing and sharing data with their colleagues as these conversations would be unlikely to occur (Datnow, Park, \& Kennedy-Lewis, 2013). They need to be afforded the leeway to make data-driven instructional decisions informed by results from common assessments (Datnow et al., 2013). This means teachers need to understand the cultural, political, and educational implications when deciding which data to use, by whom, and for what purpose (Campbell \& Levin, 2009). The data can also reinforce that the 
accountability of student learning is shared by all teachers, instructional coaches, counselors, and administrators and illustrate the areas that are deficient or exemplary (Nelson et al., 2007). Vertical Collaboration and the Transition to High School

The educational progression of a student is a continuous process that builds upon previous years where it is assumed most students are ready for the learning that should take place at their grade level (Masters, 2005). However, this progression is marred by discontinuities. With that in mind, "dropout reduction is a vertical project" (p. 2, Henderson, 2000). By virtue, this implies that combating the discontinuities that lead to dropping out of school are also a vertical project. One of those discontinuities is the transition from mid-level feeder schools to high school. For sustainable improvement the educational system needs to shift from a withinschool to between-school view (Prenger et al., 2019). Transitions from mid-level feeder schools to high school need to be studied and improved so all teachers are on the same page when managing the academic, social, and organizational factors that create such a unique and often difficult experience for students (Nelson et al., 2007; Smith et al., 2008).

Schulz and Gathier (2010) stated, "Although the awareness of the requirement of change within the school systems has been high, little is known about how sustainable intervention and innovation can be brought about within schools through collective exchange and learning beyond organizational borders" (pp. 69-70). It is widely suggested in research that communication and collaboration between mid-level feeder school and high school teachers needs to be increased and improved (Akos \& Galassi, 2004; Chapman \& Sawyer, 2001; Dorman, 2012; Egodawatte et al., 2011; Fulk, 2003; Hazel et al., 2014; Kowal, 2002; McCallumore \& Sparapani, 2010; Mizelle, 2005; Morgan \& Hertzog, 2001; Neild et al., 2007; Oakes, A., 2009; Parrish et al., 2011; Schlosser, 2015; Smith, 2006a; Smith et al., 2008). Some suggestions are more specific, 
like meeting multiple times a year with teachers across the divide (Butts \& Cruziero, 2005; Roybal et al., 2014) and using smaller designated teams to meet and then disseminate information to their native school colleagues (Dorman, 2012).

The collaborative focuses for teachers that contribute to successful transitions for students mirror those of small learning communities, vertical teams, and grade-level teams (Nelson et al., 2007). Aligning curriculum (Akos \& Galassi, 2004; Cooney \& Bottoms, 2003; Kowal, 2002; Masters, 2005; Mizelle, 2005 Mizelle \& Irvin, 2000; Morgan \& Hertzog, 2001; Parrish et al., 2011; Schlosser, 2015; Smith, 2007; Suh \& Seshaiyer, 2015), improving instruction (Fulk, 2003; Kowal, 2002; Parrish et al., 2011; Smith, 2006a), and communicating academic expectations (Akos \& Galassi, 2004; Cooney \& Bottoms, 2003; Mizelle \& Irvin, 2000; Smith, 2007, Schlosser, 2015) across the divide remained consistent with research in vertical teaming and collaboration. While collaborating that focuses on creating clear articulation of curriculum and instruction would help meet the needs of transitioning students (Mizelle \& Irvin, 2000), less than half of high school teachers have ever met with their mid-level feeder school teachers to discuss such topics (Cooney \& Bottoms, 2003; McCallumore \& Sparapani, 2010).

Outside the domains of curriculum and instruction, the collaboration between mid-level feeder school and high school teachers aligns with that of within-school cross-grade level teachers. Teachers are recommended to collaborate on behavioral expectations and issues (Cauley \& Jovanovich, 2006; Hazel et al., 2014; Oakes, 2009; Smith, 2007) as well as sharing relevant student information (Fulk, 2003) and monitoring student progress and providing proper intervention (Cauley \& Jovanovich, 2006; Cooney \& Bottoms, 2003; Ellerbrock \& Kiefer, 2014; Kowal, 2002). As Henderson (2002) stated, "Vertical teaming has given better perspective of where kids are going and where they' re coming from with regard to their learning" (p. 4). 
When understanding of students' progress along with the familiarity of curriculum in higher grade levels increases, teachers can make better informed recommendations for their students in regard to registering for the correct classes (Kowal, 2002; Nelson et al., 2007). When disruptive academic factors are alleviated, teachers can then focus their attention on easing the disruptions for students that are specific to the transition to high school. These include connecting them socially and familiarizing them to the culture and environment of a new setting (Parrish et al., 2011; Smith, 2007) and informing them of specific transition interventions that are offered (Cauley \& Jovanovich, 2006; Smith et al., 2008).

The responsibility to ensure all students transition successfully into high school is not solely placed on one institutional level but is mutual and shared throughout (Hazel et al., 2014; Mizelle \& Irvin, 2000). While the mid-level feeder school teachers strive to better prepare their students for the high school setting, receiving high schools should respond with evidence informed feedback to measure the effectiveness of their collective efforts (Cauley \& Jovanovich, 2006; Cooney \& Bottoms, 2003; Kowal, 2002). Teachers should hold each other mutually accountable in pursuing the goal of successful transitions for students (Betrand, Roberts \& Buchanan, 2006). When these ongoing intra-district efforts are made, consistency is formed by the way of common terminology, teaching methods, learning environments, assessments, and academic expectations (Henderson, 2000) that can lead to higher student achievement. Overall, the focuses of collaboration and vertical teaming between middle and high schools overwhelmingly resembles that within schools. What makes it so difficult is the unique obstacles imposed by the transition. Therefore, faculties and administrators of sending and receiving schools work to overcome those obstacles to better ensure success for incoming freshmen (Morgan \& Hertzog, 2001). 


\section{Obstacles of Collaboration}

While efficient teacher collaboration is something worth investing in, teachers work within larger systems that can oppress or empower them (Hazel et al., 2014). These systems create challenges for the collaboration movement that has been so beneficial in education. With the demands facing teachers, the lack of sufficient time is the largest obstacle facing teachers (Goddard \& Kim, 2018; Kowal, 2002; Prenger et al., 2019; Ronfeldt et al., 2015; Şahin \& Cemaloğlu, 2015). In fact, Johnston and Tsai (2018) conducted a study in which only $31 \%$ of participants reported they had enough time to collaborate with other teachers. School leaders can combat the lack of interaction by scheduling adequate time so collaboration is sustained and continuous (Akiba, 2012; Berry et al., 2008; Dorman, 2012; Gajda \& Koliba, 2008; Johnston \& Tsai, 2018; Levine \& Marcus, 2007; Parrish et al., 2011; Smith, 2007; Suh \& Seshaiyer, 2015). More specific tactics could include using built-in time collaboration during the school day, professional development days, or staff meetings to preserve teachers' time (Egodawatte et al., 2011; Kowal, 2002; Parrish et al., 2011). Another tactic would be to use technology as a method of flexible communication, which also helps with geography constraints (Egodawatte et al., 2011; Lieberman, 2000; Nicholson \& Bond, 2003). Some schools have gone as far as implementing a hybrid schedule to accommodate common collaboration times (Walker, 2018).

Another obstacle facing teachers and administration is the structure to which teachers work takes place. The organizational and geographical structure under which most schools and districts operate make it difficult for teachers to frequently meet for in-person collaboration (Akiba, 2012; Parrish et al., 2011; Prenger et al., 2019; Suh \& Seshaiyer, 2015). Many teachers, especially those in the high school setting, practice education in isolation of their peers and are generally unaware of the instructional practices others are using (Gruenert, 2005). 
The schedule and geographical constraints make it almost impossible for administrators to get all groups together for continuous collaboration (Gilmer, 2010). Therefore, school leaders conducting collaboration can plan and prioritize areas of focus to maximize the time of the participating teachers (Ronfeldt et al., 2015; Şahin \& Cemaloğlu, 2015). They can also implement formal collaborative structures and protocols with high fidelity that produce maximum efficiency that are responsive to their members (Balfanz, 2011; Berry et al., 2008; Egodawatte et al., 2011; Levine \& Marcus, 2007; Thayer et al., 2018). To create continuity, collaboration facilitators can track participation, disseminate information, and provide meaningful follow-through throughout the year (Johnston \& Tsai, 2018; Levine \& Marcus, 2007). The diffusion of ideas and concepts and their adoption into practice are often neglected without accountability from leadership (Schultz \& Geithner, 2010). At the district level, policymakers can invest in building strong communities of practice and removing obstacles that can hinder collaboration (Corcoran \& Silander, 2009; Johnston \& Tsai, 2018). They can make district level structural changes such as streamlining feeder patterns to reduce disruptions and planning adequate intra-building collaboration opportunities (Parrish et al., 2011).

Once collaborative structures have been established, teachers and school officials face social obstacles that can impede effectiveness. Success of collaboration is not a result of the quantity of the staff but the quality of their interaction (Nelson et al., 2007). The problem comes in that the varying levels of buy-in from staff can cause pockets of resistance that deters the potential progress towards meeting educational goals (Kassissieh \& Oxley, 2008). Teacher isolationism and individualism have been ingrained so deeply in the minds of some that they struggle to accept the democratic and collaborative nature of teacher teaming (Biancarosa, Bryk, \& Dexter, 2010; Gajda \& Koliba, 2008; Johnston \& Tsai, 2018). Teachers also come into a PLC 
with varying degrees of content knowledge and qualifications (Akiba, 2012) and different interpretations and understanding of goals, norms, intentions, and programs (Smith et al., 2008).

Negative interactions with peers can also create challenges within collaborative settings (Egodawatte et al., 2011). Many causes contribute to these negative interactions. A lack of communication between educators can lead to misconceptions and friction (Neild et al., 2007; McCallumore \& Sparapani, 2010). Insufficient communication can also convey varying levels of commitment (Newmann et al., 2001) and can cause members to withhold pedagogical input and information, hindering the capacity building of the team (Jacobson, 2010; Levine \& Marcus, 2010). Other cultural hinderances to collaboration is if the focus is too narrowly focused, or leadership seems too authoritarian, teachers are not as likely to take it seriously (Jacobson, 2010). Collaboration can also be intentional toward solving problems, sometimes caused by others, which can lead to teachers wasting focus by placing blame (Parrish et al., 2011). Teams may veer off in different directions or may not make good use of the minimal time they have, which can cause some to become discouraged and frustrated toward others (Jacobson, 2010). Substantial differences of collaboration may concurrently exist at the individual, team, and organizational levels in a school due to the varying social dynamics (Ronfeldt et al., 2015). As previously stated, methods exist to overcome these obstacles. Administrators and teachers can all work together to create an atmosphere of trust where teachers feel safe communicating and respectfully critique and help each other transparently (Berry et al., 2008; Şahin \& Cemaloğlu, 2015). Leaders and facilitators can conduct collaboration using strategic renewal under a healthy balance of top-down guidance and member autonomy (Jacobson, 2010; Şahin \& Cemaloğlu, 2015). Leaders can also use data and evidence to guide collaborative direction and allow teachers the leverage to make decisions based off that data (Datnow et al., 
2013). While it is an extensive undertaking to build consensus and engage a diverse faculty

(Neild, 2009), the endeavor may be worth it as teaching quality and satisfaction returns are significantly greater for those working in a better collaborative environment than those who are not (Ronfeldt et al., 2015).

\section{Benefits of Collaboration}

While many obstacles make coordinating collaboration difficult at times, its benefits are worth the investment (Gilmer, 2010). On the surface, teachers benefit from more communication, increased performance efficiency, and alleviated workload pressure (Egodawatte et al., 2011). They also have access to more reflective outlets to help manage stress, anger, and anxiety (Kowal, 2002; Ohlsson, 2013). However, teachers really benefit by building efficacy when their schools commit to achieving a coherent ongoing focus of improving teaching quality collaboratively (Egodawatte et al., 2011). Teachers' collective learning through high-functioning teams increases their collective efficacy regarding student achievement and can create positive change in their school (Donohoo \& Katz, 2019; Lee et al., 2011). Their collective instructional capacity grows because of their exposure to the different practices of their peers (Crawford, 2011; Walker, 2018), along with the constructive critiques exchanged about their own methods (Birkhead et al., 2017; Goddard et al., 2015; Moolenaar et al., 2012; Prenger et al., 2019). These interactions strengthen teachers' commitment to their students and continuously propels them to make purposeful adjustments to their own practice to increase student achievement (Donohoo \& Katz, 2019; Goddard \& Kim, 2018; Lieberman, 2000; Ronfeldt et al., 2015). This quality collaboration produces an environment where teachers exhibit shared accountability and collective responsibility for student learning (Ronfeldt et al., 2015). 
The quality of collaboration influences teaching quality, which in turn influences student achievement (Moolenaar et al., 2012; Ronfeldt et al., 2015). There is a positive link between student learning with teacher cooperation and collective responsibility for student learning (Corcoran \& Silander, 2009). Ismail et al. (2018) and Goddard et al. (2015) both found that teacher collaboration is a partial mediator between strategic instructional leadership and teaching quality. Overall, schools that have a comprehensive high-quality teacher collaboration program in place experience unprecedented gains in student achievement (Gajda \& Koliba, 2008). In fact, teacher small learning communities are one of the most powerful longitudinal student achievement predictors (Egodawatte et al., 2011) and are more impactful than individual teacher attributes (Berry et al., 2009). A collaborative school culture is not only positively correlated with student achievement (Gruenert, 2005), teacher attrition is reduced especially in high needs schools that need quality teaching the most (Berry et al., 2009).

The quality of teacher collaboration is positively correlated to student achievement gains (Goddard et al., 2015), especially when the collaboration focuses on instruction and assessment (Ronfeldt et al., 2015). It is important to note that reverse causality can occur as increased student progress may produce improved collaboration (Ronfeldt et al., 2015). Results from student standardized tests show that better teacher collaboration relates to increases in achievement specifically in math and reading (Ronfeldt et al., 2015; Slavin, Lake, \& Groff 2009). A case study of the Aldine Independent School District in Houston, Texas, Henderson (2000) found collaborative efforts along with an agreed upon learning expectation increase among staff resulted in a five year increase in student achievement on state test scores by an average of approximately $26 \%$ in reading, writing, and mathematics. Their dropout rate also decreased from $7.3 \%$ to $1.8 \%$ (Henderson, 2000). Jackson and Bruegeman (2009) quantified a 
teachers' added value through student test score gains and found that $20 \%$ of a teacher's added value was attributable to shared expertise.

Overall, the research suggests that a promising path to educational improvement is through increasing the quality of instructional team collaboration (Ronfeldt et al., 2015). When it comes to teacher collaboration, The National Staff Development Council (2005) as cited by Gajda \& Koliba (2008) summarizes it best:

They consider teacher collaboration the foundational element of any successful professional development effort and asserts that staff development that improves learning for all students organizes adults into learning communities. Consensus exists among school restructuring advocates that teacher collaboration is one of the most essential, if not the most important, requisite for achieving substantive school improvement and critical student learning outcomes. (p. 2) 
SECTION FOUR: CONTRIBUTION TO PRACTICE 


\section{Plan for Dissemination of the Practitioner Contribution}

Creating IPC between teachers across the transition to high school is a new concept in research so the rationale of the researcher is to expose others to the issue and how it can be beneficial for students. The low response rate of this study made it inconclusive to alone be the base of a presentation. An implication is that many school districts do not have a structure or protocol in place to create vertical collaboration between teachers at different grade levels in separate geographical locations. Yet, a substantial amount of research exists pertaining to the transition from mid-level feeder schools to high school that includes many recommendations that have shown to increase student achievement and ease the disruption many students face. Further research needs to be conducted to see if this has a positive effect on student achievement that requires the attention of school leaders and staff. Therefore, this presentation will be aimed at offering suggestions to create IPC across the divide using recommendations from research coupled with key statistics from this study and recruit potential participants for further studies.

The time for dissemination will begin soon after the completion of the study. The goal for presenting would be at a Missouri Association of Secondary School Principals (MASSP) Conference. At this conference, audience members will be given a pamphlet to guide them through the presentation as seen in Figure 4.1. To add breadth to the presentation, upon completion of this study, further research will be conducted to discover success stories of high schools collaborating with their mid-level feeder schools. At this presentation, contacts and online means of communication will be shared to network different ideas and results of collaborative exploration at different school districts. Regardless of the means of dissemination, it is imperative that the language of the presentation or publication is translated to best speak to its intended audience. 

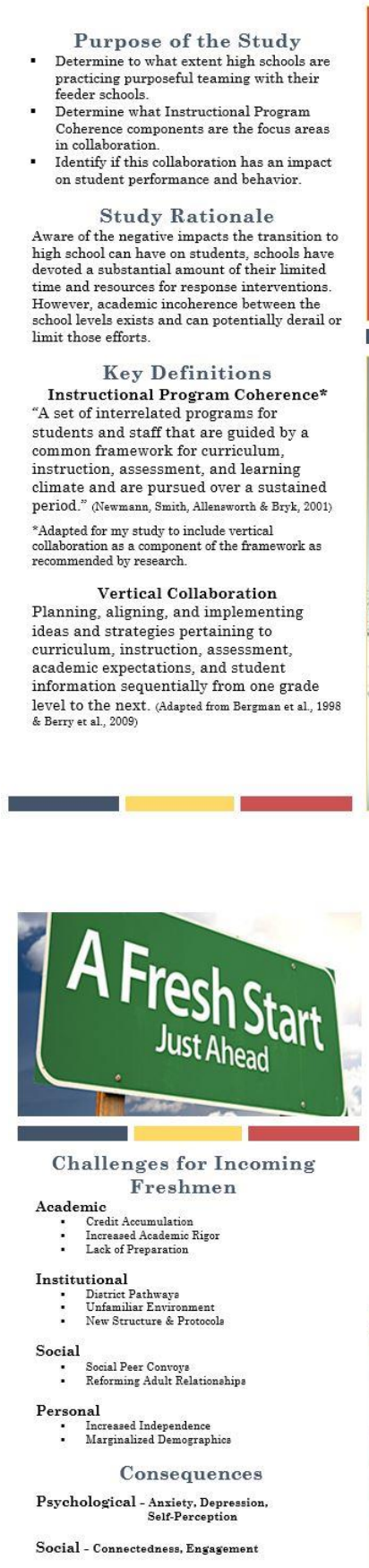

Behavioral - Discipline, Absenteeism

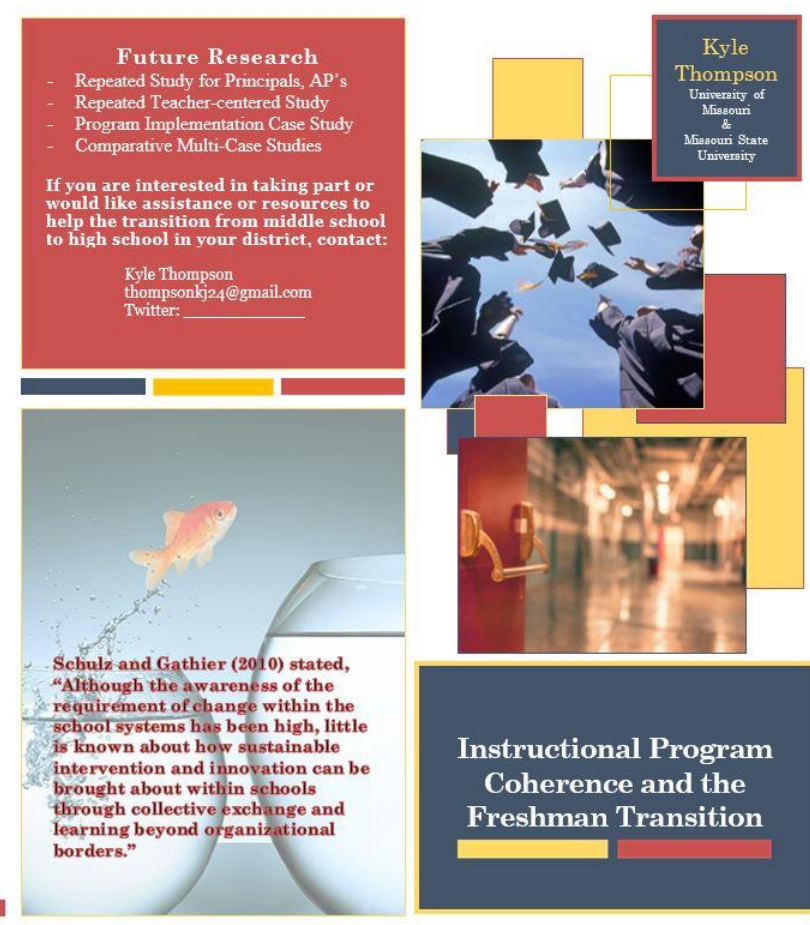

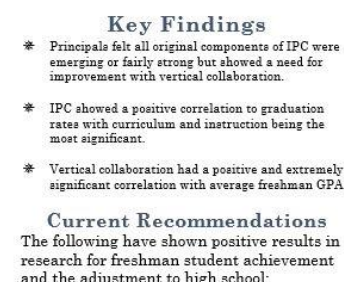

and the adjustment to high school:

Freshman Small Learning Communities
Teacher/Student Freshman Mentoring

: Summer Transition Program Mentorin

Preparatory Freshman Seminar Class
Freshman Orientation \& Open House
Early-risk Identification \& Intervention
A "Zero Hour" or Intervention Period

: Upper-Classmen Q\&A A

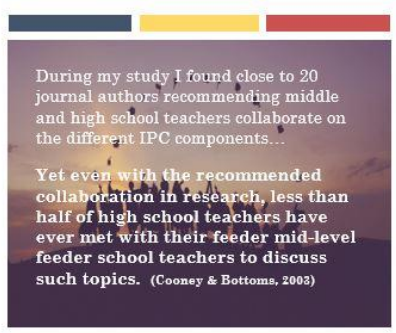

Schools with fully implemented freshman intervention programs have dropout rates one-third the size of those schools without these systems in place. - Kennelly \& Monrad, 2007

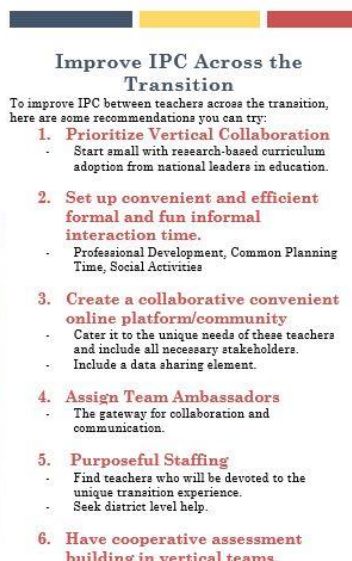

Figure 4.1. Brochure to guide attendees of a MASSP Conference. The focus of this presentation will be to share recommendations for the freshman transition based off the results and research, and recruit participant schools for future studies. 
Besides trying to reach the broadest audience possible, the rationale for this plan of dissemination is to reach decision makers in school districts. Part of the reason this type of study was conducted is that to introduce an issue that needs the focus of administrators, evidence backed by scientific numerical data grabs attention. The timing of this plan for dissemination is ideal as it aligns with the Missouri PLC School Improvement Initiative (DESE, 2020c). The earlier this information is presented to administrators, the more time they must determine if it is an area they should focus on and start planning their PLC structures and objectives accordingly. However, since the low response rate made this study too inconclusive to submit to a journal, the focuses of this presentation will be to build awareness, pique interest, and attract recruitment for further publication. During this conference, information will be gathered by the presenter that could inform future studies. For example, maybe a principal will attend whose district already has an effective means to promote cohesion vertically between buildings and could be the focus of a future case study by the researcher.

The following is the PowerPoint presentation that will be guiding the presentation at the MASSP Conference. The first focus of the presentation will be on the key finding that shows a significantly strong moderately positive correlation between vertical collaboration and average freshman GPA. The second focus will be on current freshman transition recommendations for intervention followed by researcher recommendations that specifically target building IPC between teachers across the transition. 

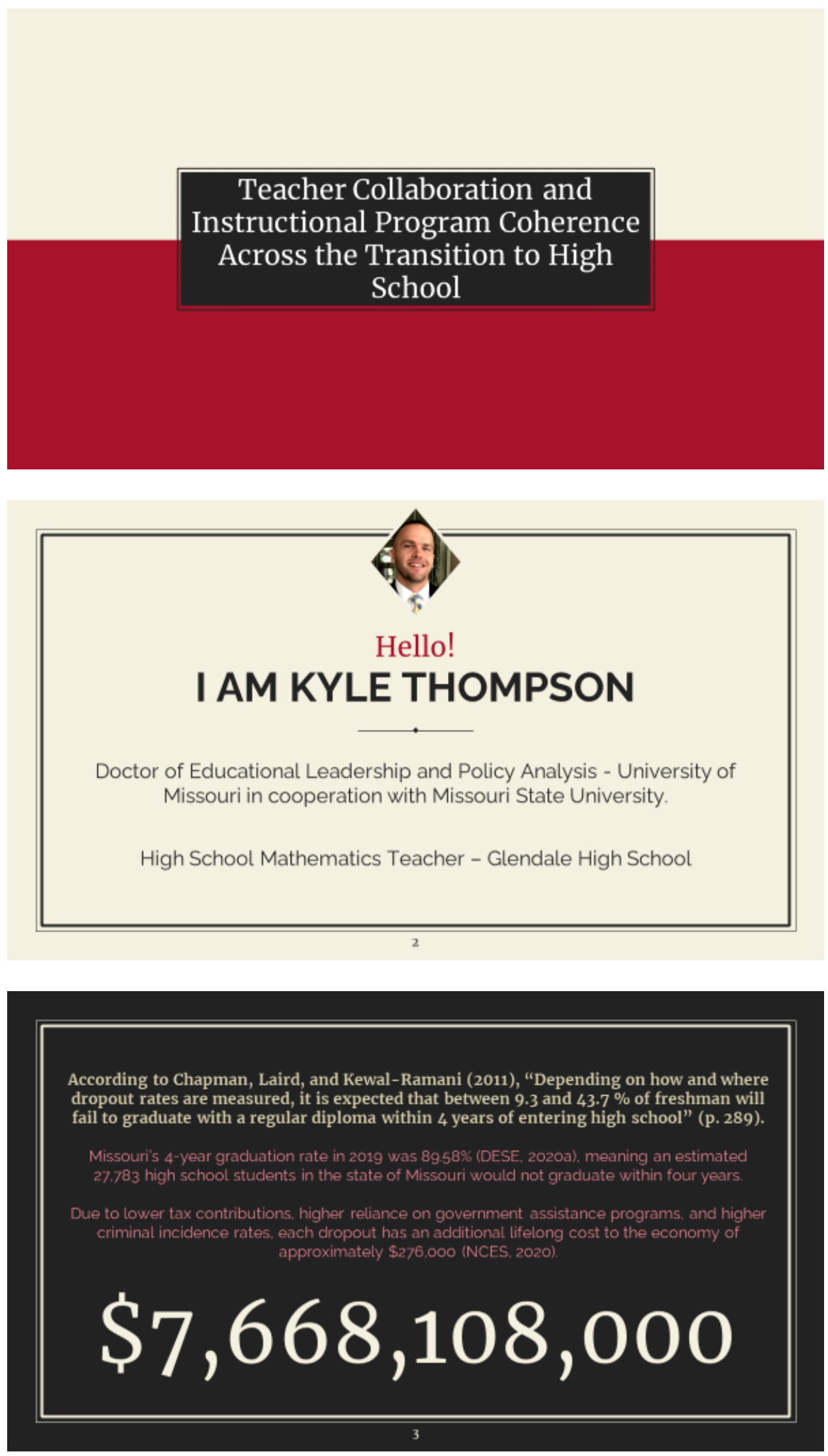


\section{Statement of Problem}

A poor transition from mid-level feeder schools to high school contributes to dropping out

Schools have responded to these findings by investing greatly in various transitional programs and interventions.

However, the academic incoherence between mid-level feeder school and high school teachers can derail these efforts.

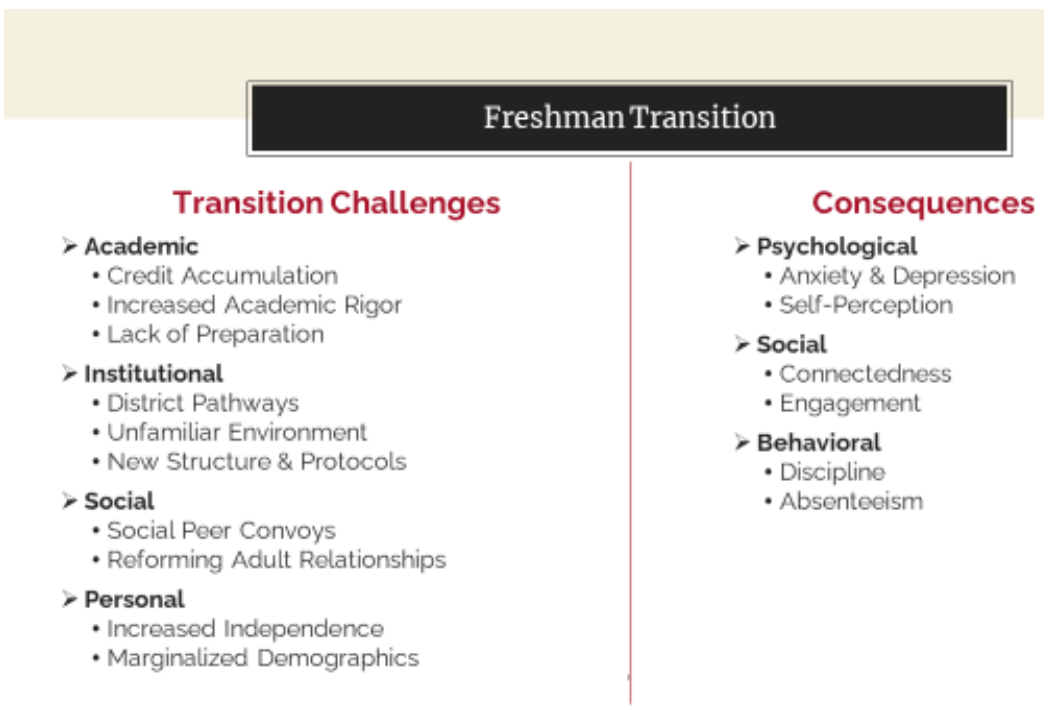

(6)

\section{Purpose of the Study}

There are two purposes of this study:

1. Determine to what extent high schools are practicing purposeful vertical teaming with their mid-level feeder schools.

2. Identify if this collaboration associates to high school student achievement using various positive and negative measures. 


\section{Research Questions}

1. As perceived by high school principals, to what extent is IPC utilized in Missouri secondary schools?

2. What is the relationship between IPC (as perceived by high school principals) among mid-level feeder school and high school teachers and student achievement as measured by:
a. Graduation rate?
b. Missouri End-of-Course Exams?
c. Freshman grade point average (GPA)?
d. Freshman attendance?

7

Instructional Program Coherence(IPC)

Definition:

A set of interrelated programs for students and staff that are guided by a common framework for curriculum, instruction, assessment, and learning climate and that are pursued over a sustained period"

4 Domains:

Curriculum Instruction

Assessment Academic Climate

Adapted to include:

Vertical Collaboration

3 Prevaling Conditions:

1. A common instructional framework with specific expectations and strategies guides teaching and assessment.

2 Staff working conditions support implementation of the framework.

3. The school allocates resources such as funding, materials, time, and staff assignments to advance the common framework and to avoid diffuse, scattered improvement efforts

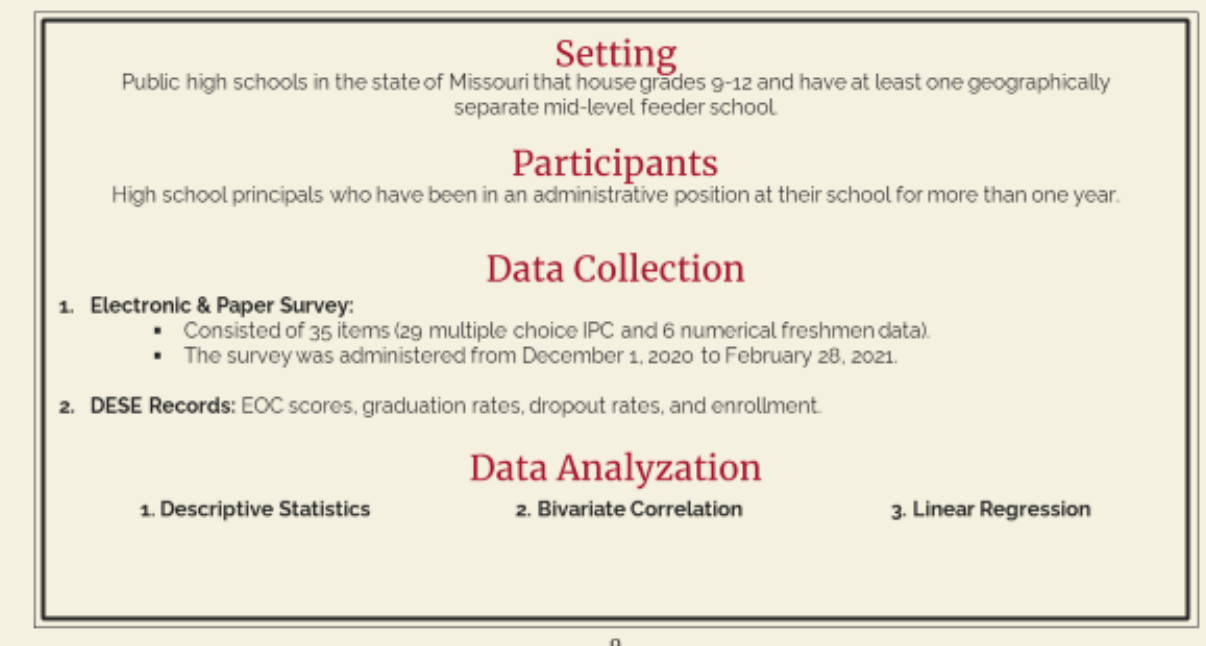




\section{Survey Scale}

The scale was adapted from Newmann et al.'s (2001) IPC 4-point Likert scale as follows:

$1=$ Not At All or Does Not Exist

2 = Somewhat or Needs Improvement

3 = Most or Emerging/Proficient

$4=$ To Great Extent or Excelling

\section{Key Findings}

- Principals viewed overall IPC as Emerging/Proficient with a 3.07 out of 4 measure.

- Emerging/Proficient IPC component means were:

- academic climate (3.23)

- curriculum(3.17)

- instruction (3.16)

- assessment (3.09)

\section{Results}

\section{Vertical collaboration (VC) was the only score needing improvement at 2.70}

> 39 less than the next lowest component

- Almost three times the range of the other four components

> VC dragged the overall IPC mean below the mean of all the other means

- Overall IPC would be 3.1625 without the vertical collaboration component 


\section{Vertical collaboration showed the highest positive correlation with the average freshman GPA.}

Moderate positive correlation (542)

Most statistically significant $(p=.001)$

Could vertical collaboration be a key contributor to keeping freshmen on track for graduation?

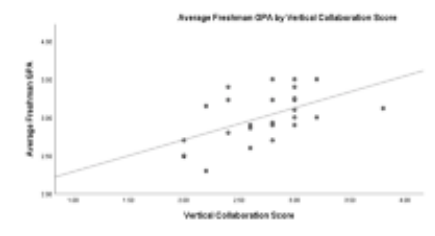

13

Additional Key Findings

The following were additional significantly significant measurements from the study $(p<.05$ level).

I IPC components instruction $(R=.378 ; p=.033)$ and the curriculum $(R=.384 ; p=$ .030 ) had a moderately positive association with the graduation rate.

- Assessment and academic climate shovred similar associations with graduation rates that were extremely close to statistically significant.

Academic climate showed a statistically significant moderately positive correlation with the Algebra 1 EOC scores $(R=.435 ; \mathrm{p}=.013)$.

14

\section{Future Research}

1. A replicated study focused on just vertical collaboration.

- Research calls for vertical collaboration and it was the lowest scored component of the current stucly.

2. A replicated study with teachers surrounding the transition as participants.

- The perceptions of teachers could differ from those of their principals.

3. A case study on a school district that employs or will begin to employ vertical collaboration strategies between middle and high schools.

- This would include a qualitative portion for depth on measures taken to promote vertical collaboration and IPC

4. A longitudinal study with a school district initiating new vertical collaboration programs to bring IPC between their teachers and schools.

- Compare student achievement scores from before and after 


\section{Recommended Interventions in Research}

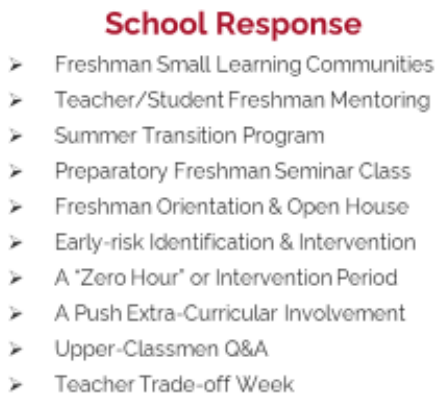

"Schools with fully implemented freshman intervention programs have dropout rates one-third the size of those schools without these systems in place."

16 - Kennelly \& Monrad, 2007

\section{Researcher Recommendations}

Here are some recommendations to improve IPC between teachers across the transition to high school:

1. Prioritize vertical collaboration

2. Start small with trusted research-based whole subject curriculum adoption

3. Set up effective formal and (fun) informal interaction time

4. Create a collaborative convenient online platform/community

5. Assign team/department ambassadors to work across the transition

6. Purposeful staffing

7. Have cooperative assessment building in vertical teams.

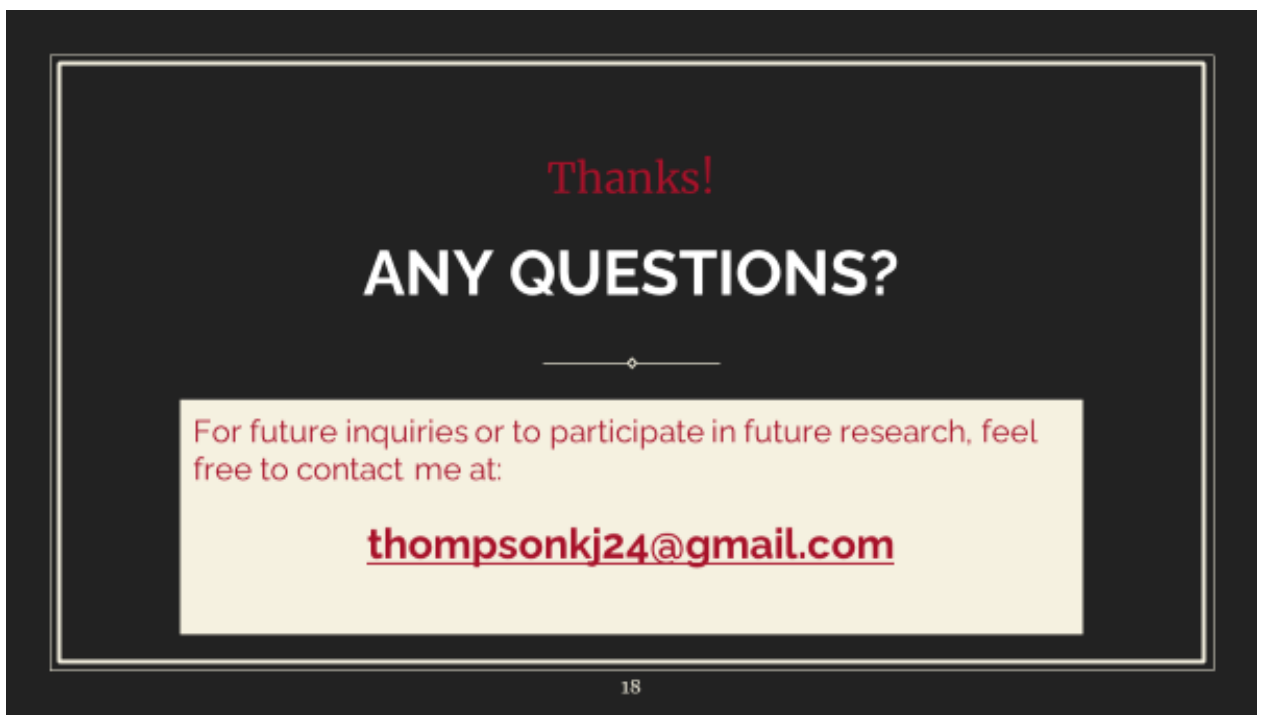


SECTION FIVE: CONTRIBUTION TO SCHOLARSHIP 
Vertical Collaboration and Instructional Program Coherence Across the Transition to High

School

Kyle J. Thompson and Kim K. Finch

University of Missouri

Author Note

Kyle J. Thompson, Educational Leadership and Policy Analysis, University of Missouri;

Kim K. Finch, College of Education, Missouri State University.

This research has no conflict of interest to report.

Correspondence concerning this research should be addressed to Kyle J. Thompson,

Educational Leadership and Policy Analysis, University of Missouri, Columbia, MO 65211.

Email: thompsonkj24@gmail.com

To be submitted to: The Clearing House 


\begin{abstract}
This study explores to what extent high school principals perceive instructional program coherence (IPC) and vertical collaboration occur with their geographically separate feeder schools. Survey data was analyzed using descriptive statistics, bivariate correlations, and linear regression. Findings indicate principals perceive strong IPC, but vertical collaboration needs improvement. IPC components curriculum and instruction positively influenced graduation rates, academic climate positively influenced EOC Algebra 1 scores, and vertical collaboration positively associated with freshmen average GPA. A limitation was the low response rate.
\end{abstract}

Key Words: instructional program coherence, vertical collaboration, vertical teaming, collaboration 
Educational patterns show the transition to high school is arguably the most impactful proximal context in a student's long-term life trajectory. The ninth grade has the highest enrollment of any grade due to retention, but also loses the most students to dropping out by the end of the year (Cohen \& Smerdon, 2009; Emmet \& McGee, 2012). Roughly 25\% to 50\% of students entering the ninth grade will fail to receive a diploma (Casillas et al., 2012), contributing to the dropout crisis plaguing the United States. The increase in dropout rates stems from the deficient academic progress many students face by the end of their freshman year (McCallumore \& Sparapani, 2010). Freshmen face credit accumulation requirements for the first time in their education when academic achievement is required for grade advancement (Nield, 2009). Coinciding with higher stakes is the increase in both academic rigor (Brown \& Seely, 2010) and autonomy (Chapman \& Saywer, 2001). This combination can cause a damaging disruption to a pupil's learning process, especially with deficient preparation.

Roughly $40 \%$ of students experience struggles due to the transition to high school that contribute to negative academic outcomes (Akos \& Galassi, 2004). In addition to the academic change, students face contextual and social disruptions. They must navigate through larger unfamiliar buildings and adapt to new protocols and responsibilities (Gentile-Genitty, 2009). Social convoys also change due to a larger and more diverse school population (Langenkamp, 2009). Transitional difficulties may also cause psychological hardships that students must simultaneously navigate (Benner, 2011; Uvaas \& McKevitt, 2013). The life trajectory of each adolescent may depend on their ability to overcome the distress this transition may cause. Recognizing this challenge, districts have responded by allocating inordinate amounts of resources to deploy various interventions from comprehensive school reform models to freshman 
transition programs (Benner, 2011; Emmett \& McGee, 2012; Kennelly \& Monrad, 2007;

McIntosh \& White, 2006; Roybal, Thornton \& Usinger, 2014).

Even with extensive dedication to interventions, the academic incoherence between middle and high school teachers could derail the effectiveness of a district's efforts. Educators could compensate with a deliberate effort to build Newmann, Smith, Allensworth and Bryk's (2001a, 2001b) concept of instructional program coherence (IPC), defined as "a set of interrelated programs for students and staff that are guided by a common framework for curriculum, instruction, assessment, and learning climate and are pursued over a sustained period" (p. 299). Within this concept, schools allocate resources to wide-range reform of shared academic vision and consistent means to achieve it (Newmann et al., 2001). Cross-grade collaboration or vertical teaming (VT) between teachers is imperative to increase IPC. While research shows teacher collaboration in almost any form has a positive effect on student achievement (Ronfeldt, Farmer, McQueen, \& Grissom, 2015; Moolenaar, Sleegers, \& Daly, 2012), vertical collaboration is very challenging due to the constraints of time, geography, and resources (Bergman \& Calzada, Lapointe, Lee \& Sullivan, 1998; Berry, Daughtrey \& Wieder, 2009; Vangrieken, Dochy, Raes, \& Kyndt, 2014). Preferably, an IPC framework with a component for convenient vertical collaboration would already be embedded in the district structure (Berry et al., 2009; Gagda \& Koliba, 2008; Vangrieken et al., 2014).

\section{Statement of the Problem}

According to Chapman, Laird, and Kewal-Ramani (2011), "Depending on how and where dropout rates are measured, it is expected that between 9.3 and $43.7 \%$ of freshman will fail to graduate with a regular diploma within 4 years of entering high school” (p. 289). A partial contribution to this outcome is a chain reaction spurred by barriers students face caused by the 
transition from their mid-level feeder school to high school. Comprehensive research on those barriers spans across academic, structural, social, and personal domains (for summary see Benner, 2011). Reactionary research has focused on schools' responses to these findings with various transitional programs (Emmett \& McGee, 2012; Smith, 2006b) primarily at the student and organizational levels. The teacher focus within this literature is limited to focusing on stronger student-teacher relationships (Wentzel, Tomback, Williams \& McNeish, 2019) and horizontally collaborating to lessen academic and behavioral issues (Emmett \& McGee, 2012).

Converging with the research on the student transition to high school is the concept of IPC in schools. While it is evident that increased cohesion has an overall positive effect on student achievement (Madda, Halverson \& Gomez, 2007; Newmann et al., 2001), the pattern in student achievement suggests that IPC suffers the same disruption from the transition from middle to high school that students do. The IPC framework's aim for entire school cohesion (Newmann et al., 2001) aligns with the ideal of many all-inclusive school reform programs that cater to incoming freshman. However, variables exist in the transition to high school that make its impact unique to the rest of the educational progression. The universal nature of IPC framework then becomes a shortcoming because its exclusivity to specific areas of need, like the transition from middle to high school. Newmann et al. (2001) recognized that different areas of need require specific attention that should adhere coherently with the rest of the institution.

Vertical teacher collaboration to increase IPC across such a unique impactful disruption in a student's educational progression provides the problem of focus of this study. A lack of research exists that demonstrates if districts are using teacher-to-teacher collaboration vertically between separate buildings and if this collaboration impacts student achievement. Also, while studies suggest that mid-level feeder school and high school teachers collaborate (Berry et al., 
2009; Dorman, 2012), researchers do not elaborate on the recommended focus of the collaboration, nor what these collaborative practices should entail. Before specifying what teachers prioritize in collaboration, research needs to be done to determine if it is happening and how it affects students' initial success in high school.

\section{Purpose of the Study}

The differences between the middle and high school settings along with unforeseen challenges converge to contribute to the distress of students making the transition into high school. The inability to overcome these challenges can lead to consequences that can derail students' life trajectory. Schools have offered specific transitional programs to aid students in this change. The success of these transitional programs requires collaboration between each building's personnel (Smith, 2006b). Unfortunately, the disjoint structure and relationships across this transition hinder academic coherence between staff. School personnel across the divide need to devote specific attention to create IPC to ease the academic disruption. However, while IPC is positively associated with student achievement (Newmann et al., 2001), its conceptual framework does not articulate the practical means to produce it. Without effective collaborative strategies in this demanding environment, these teachers will remain incoherent to what academic alignment is needed to alleviate the academic disruption for students during their transition to high school.

There are two purposes of this study. The first is to determine to what extent high schools are practicing purposeful VT with their mid-level feeder schools from the perspective of high school principals. This will also include how schools prioritize the four components of IPC within their collaboration at both levels. The second purpose will be to identify if this collaboration has an impact on different forms of high school student achievement. 


\section{Research Questions}

The research questions (RQ) guiding this study were:

1. As perceived by high school principals, to what extent is IPC utilized in Missouri secondary schools?

2. What is the relationship between IPC (as perceived by high school principals) among mid-level feeder school and high school teachers and student achievement as measured by:
a. Graduation rate?
b. Missouri End-of-Course Exams?
c. Freshman grade point average (GPA)?
d. Freshman attendance?

3. What is the relationship between IPC (as perceived by high school principals) and high school student failure as measured by:
a. Dropout rate?
b. Freshman course failure rate?
c. Freshman out-of-school (OSS) suspension rate?

\section{Key Terms}

In studying collaboration between teachers across the mid-level feeder school and high school transition, many common terms appear throughout research. For clarity, the following is a list of key terminology guiding this study:

- Mid-level Feeder Schools consist of schools of any structure that feeds into a high school that starts at grade 9 . 
- Collaboration - "interaction with colleagues that focuses on improving classroom instruction, curriculum, and supports for students." (Johnson \& Tsai, 2018, p.3)

- Vertical Collaboration - Planning, aligning, and implementing ideas and strategies pertaining to curriculum, instruction, assessment, academic climate, and student information sequentially from one grade level to the next from grades prekindergarten through grade 12 (Bergman et al., 1998; Berry et al., 2009).

- Vertical Teaming - people from different levels within an organization who are committed to a common purpose, performance goals, and approach for which they hold themselves mutually accountable to achieve the desired objective of improving student learning by developing the efficacy of educators (Crawford, 2011).

\section{Significance of the Study}

Extensive research suggests collaboration between mid-level feeder school and high school teachers eases the disruptive process incoming freshmen face because of the transition (Brown \& Seely, 2010; Chapman \& Sawyer, 2001; McIntosh \& White, 2006; Oakes, 2009; Smith, 2006a). However, a gap exists if teacher collaboration between feeder and high school teachers is taking place, what the focus of this collaboration is, and if this collaboration influences student success. The IPC provides a conceptual model, but this study provided insight crucial in clarifying the academic elements teachers can stabilize to improve the learning process for students entering high school. The demands on teachers require them to be more resourceful with their time and efforts, meaning teachers bordering the transition must creatively navigate the unique barriers it creates. This study provided insight to determine if vertical collaboration practices across this divide need to be pursued. 


\section{Instructional Program Coherence}

This study's guiding conceptual framework of IPC was pioneered by Newmann et al. (2001) as, "a set of interrelated programs for students and staff that are guided by a common framework for curriculum, instruction, assessment, and learning climate and that are pursued over a sustained period" (p. 297). The framework consists of four major domains. Curriculum relates to the sequential alignment of academic content that, when properly executed, can improve student achievement (Squires, 2012). Instructional coherence applies to aligning the pedagogical means of achieving a united academic goal (Madda et al., 2007). Common summative and formative assessments allow teachers to analyze results across classrooms and collaboratively learn how to improve their instruction or intervention based on their findings (Jacobson, 2010). Finally, it is well documented that a misalignment of expectations and rigor exists between mid-level feeder school and high school staff (Chapman \& Sawyer, 2001; Cohen \& Smerdon, 2009; McCallumore \& Sparapani, 2010; Smith, 2006). Creating a mutual consensus of these learning expectations would be considered a way to improve academic climate.

Three prevailing conditions are evident in IPC schools (Newmann et al., 2001). The first is that leadership and work on the four components progresses toward a common framework. Two goals of collaboration are to increase both democratic unity and teacher efficacy (Stosich, Bocala, \& Forman, 2018). Achieving these would be implementing activities that create a mutual understanding of IPC requirements at both the eighth and ninth grade levels to mitigate the academic disruption students experience from the transition (Mizelle \& Irvin, 2000). The second condition is ensuring working conditions are conducive toward implementation of the framework (Newmann et al., 2001). Disparate organizational structures provide barriers to collaboration that schools need to bridge through innovative strategic management and common 
planning time (Smith, Akos, Lim, \& Wiley, 2008). The third condition is that resources are appropriately applied to attain and advance that framework, pertains to the technology tools and training that can be used to improve coherence (Newmann et al., 2001). Research shows that schools are allocating resources toward transitional interventions (DeLamar \& Brown, 2016; Herlihy \& Kennelly, 2007; Kennelly \& Monrad, 2007) and selectively positioning the right teachers within these grade levels (Ellerbrock \& Kiefer, 2014; McIntosh \& White, 2006). It is those teachers' obligation to provide academic alignment to the best of their abilities to help freshmen reach optimal academic achievement.

A limitation of IPC is that it is institutionally focused while this study will focus on the transition from mid-level feeder schools to high school. However, Newmann et al. (2001), who pioneered the IPC concept cautioned, "The pursuit of greater program coherence must respond to appropriate forms of differentiation and be receptive to new or altered programming for staff and for students when clearly necessary" (p. 313). The entrance to high school is a disruption that requires specific interventions to ensure a successful transition for students.

\section{Literature Review}

While individual experiences vary through the transition, common educational, institutional, social, and personal factors contribute to create stress and obstacles that put these students in academic jeopardy (Galla, Tsukayama, Park, Yu. \& Duckworth, 2020). Since the focus on this study was IPC, this review examined and connected those academic barriers unique to the freshman transition. It also examined the role of collaboration and vertical teaming in creating student success in academic achievement. 


\section{Academic Causal Transition Factors}

The disruption caused by the transition to high school has an almost universal negative effect on student academic achievement (Alspaugh, 1998; Benner, 2011; Cohen \& Smerdon, 2009; Kennelly \& Monrad, 2007; Neild et al., 2008; Smith et al., 2008). The academic factors most associated with this decline are the credit accumulating requirements coupled with the increased rigor students experience when entering high school.

Credit Accumulation. An immediate stressor for students entering high school is that because the goal is graduation, they start to attribute future opportunities outside of school to academic success in school (Chapman \& Sawyer, 2001). Part of this is because high school requirements differ from that of their mid-level feeder school (McCallumore \& Sparapani, 2010). Students enter a credit accumulation requirement system, where they must earn passing grades in courses to advance for the first time and ultimately graduate (Emmett \& McGee, 2012; Fulk, 2003; McCallumore \& Sparapani, 2010; Neild et al., 2008, Smith et al., 2008).

Many students are unaware of the newly required course success as it is different from the promotion methods in their mid-level feeder school where they learned a system to just "get by" (Neild, 2009). Distracted with acclimating to the new environment and higher rigor,

freshmen usually realize the importance of passing their courses after that opportunity is gone (McCallumore \& Sparapani, 2010; Neild et al. 2008; Smith et al., 2008). Making it worse is that it can take a year for students to recover to the achievement level they were at before the transition (Akos \& Galassi, 2004; Alspaugh, 2001; Somers, Owens, \& Piliawsky, 2009; Uvaas \& McKevitt, 2013). Those students' reality then becomes that pursuing their chosen career path is much more difficult because necessary skills and knowledge are much harder to obtain (Cooper \& Liou, 2007). This is not due to a lack of intellect or motivation, but rather their inability to 
effectively navigate and negotiate the educational system (Cooper \& Liou, 2007). Not realizing the importance of classroom success early enough is one reason course failure and dropout rates are higher in the ninth grade than any other grade (Smith et al., 2008).

When students do not earn enough credits for promotion in the allotted time, they go into credit recovery mode, or they are "off track" (Neild, 2009). Failed courses must be retaken, and promotion to the next grade, and ultimately graduation, will be deferred until credit requirements are met (Neild, 2009; Mizelle \& Irvin, 2000; Smith et al., 2008; Uvaas \& McKevitt, 2013). That is why course failure is a better indicator of dropping out than low test scores from mid-level feeder schools (Balfanz et al., 2007), and why it is magnified during the freshman year (McKee \& Caldarella, 2016; Roybal et al., 2014). Data show that once students successfully reach tenth grade, they are likely to graduate high school (McCallumore \& Sparapani, 2010). Allensworth and Easton (2005) found that $81 \%$ of Chicago Public School students that ended their freshman year on track graduated within four years with an additional $4 \%$ finishing in their fifth. Since this relationship does not change when additional factors such as race, gender, SES, and prior achievement were considered, the correlation between being on track at the end of the first year of high school and graduation rates is very strong (Allensworth \& Easton, 2005). Success or failure in the ninth grade sets the tone for the rest of high school (McIntosh \& White, 2006).

Increased high school rigor. A noticeable disparity in rigor exists between high school and mid-level feeder school classes for both high and low achieving students (McCallumore \& Sparapani, 2010). Three out of four freshmen report their classes are much more difficult than their mid-level feeder school classes (Chapman \& Sawyer, 2001). Parents and students are aware of the rigor increase as they state academic success and increased demands (e.g. homework) as a top concern (Akos \& Galassi, 2004; Uvaas \& McKevitt, 2013; Wentzel, et al., 
2019). Ninth graders report they are held to higher standards and expectations by their teachers through larger workloads and increased demand for their time to be spent on independent schoolwork (Galla et al., 2020; Mizelle, 2005). They also report more severe consequences for not completing work in high school than ever before (Ellerbrock \& Kiefer, 2013). Credit accrual makes students' perceptions of goals in high school more performance oriented and less mastery oriented than it was in their mid-level feeder school (Anderman \& Midgley, 2004).

The inequity in rigor along with the well documented decline in achievement scores across the transition to high school often leads to an examination of the preparation students receive in mid-level feeder schools to be successful in high school. Unfortunately, students who earned decent subjective grades but were often unchallenged and those who were at-risk of failure, regularly come to high school unprepared and their lack of skills and mastery knowledge finally catches up with them (Neild et al., 2007; Neild, 2009). As Emmet and McGee (2012) pointed out, "Without the foundational skills, access to academic content becomes limited and the credit accrual system that drives the process of high school graduation becomes a difficult obstacle to overcome" (p. 75). According to the research of Cooney and Bottoms (2003), 51\% of high school teachers feel incoming students do not possess the necessary background knowledge when they enter high school. Struggling students often come to high school with weak math and reading skills yet are expected to pass college preparatory classes they see for the first time (Brown \& Seely, 2010; Neild \& Balfanz, 2006; Neild, 2009).

Neild (2009) illustrated the lack of preparation problem through the 2008 National Mathematics Advisory Panel recommendation that Algebra students need to have previously acquired mastery of fractions, decimals, and integers. Students without those skills may not be able to acquire them from their Algebra course due to the already limited time to complete the 
required curriculum (Cohen \& Smerdon, 2009), and school teachers may also lack the expertise to teach content for the earlier grades (Neild, 2009). The predicament for high school teachers is that new reforms repeatedly call for more rigor (Cohen \& Smerdon, 2009). But Corcoran and Silander (2009) contended, "at the same time that high schools are being asked to offer more rigorous preparation for larger numbers of students, they are also being asked to ensure that all or almost all students meet rising academic standards and that dropout rates decline" (p. 158). This demonstrates why higher eighth grade achievement scores were predictive in freshmen success (McKee \& Caldarella, 2016; Neild, 2009), graduating on time (Lovelace, Reschly, \& Appleton, 2017), and college readiness (Huerta, Watt \& Butcher, 2013). Academic press refers to the extent which the educational experience emphasizes high academic standards is also a major predictive concept of student effort and achievement because academic and behavioral problems students have in mid-level feeder schools rarely self-correct (Balfanz et al., 2007).

A common suggestion in research is for feeder schools to increase the rigor in their curriculum and incorporate classroom practices that will prepare students for the learning they will experience in high school (Chapman \& Sawyer, 2001; Cohen \& Smerdon, 2009; Huerta et al., 2013; Smith, 2006a). Neild (2009) agreed and stated, "the problem lies with students' weak preparation for high-school work or misconceptions of the diligence required to earn a high school diploma, or both" (pp. 59-60). Mid-level feeder schools should provide a challenging and rigorous curriculum that provides the necessary mastery knowledge for students to successfully build upon throughout high school (Cohen \& Smerdon, 2009; Mizelle, 1995; Nelson, Fairchild, Grossenbacher, \& Landers, 2007; Parrish et al., 2011; Smith, 2006a). Yet, content should not be the only focus of feeder schools in preparing students for high school. 
Effective mid-level feeder school teachers are intentional and take great pride in promoting well-equipped students to high school by setting and enforcing high classroom standards and expectations that promote independence and responsibility of students in their learning (Cooney \& Bottoms, 2003; Dedmond, Brown \& LaFauci, 2006; Mizelle, 2005; Parrish et al., 2011). Cauley and Jovanovich (2006) reported that ninth graders agreed stating, "they would have been better prepared for high school had their middle school teachers challenged them more, held them responsible for their learning, and taught them strategies to learn on their own" (pp. 17-18). It is essential for mid-level feeder school teachers to prepare students for the rigors of high school in every academic facet they can (McCallumore \& Sparapani, 2010).

\section{Vertical Collaboration}

While schools have made numerous efforts to quell the disruptiveness the transition to high school causes, there exists an academic disconnect vertically between mid-level feeder school and high school teachers. This disconnect can make many of the school district's interventions fail to reach their full potential. Substantial literature exists on collaboration throughout a school district to improve IPC and subsequent student achievement. The following outlines the general structure and focuses of collaboration and continues with an examination of the current research on vertical collaboration and teaming between mid-level feeder school and high school teachers.

\section{Professional Learning Communities and Teacher Collaboration.}

Quality teacher collaboration has increasingly been shown to have substantial positive effects on school improvement through teachers taking shared accountability toward continuous improvement (Ronfeldt et al., 2015). Johnston and Tsai' s (2018) definition of collaboration incorporates Newmann et al.' s (2001) key elements of IPC. It states teacher collaboration is an 
interaction between colleagues focused on improving and enhancing instruction, curriculum, assessment and supports for students (Johnson \& Tsai, 2018). Teachers often rely on each other to assist in their professional development before any other resource. Berry et al. (2009) found teachers turn to peers for initial support $68 \%$ percent of the time. Knowing this, schools can capitalize on that interaction by formalizing and structuring teacher collaboration to make it more intentional and utilizing the variety of staff expertise at their disposal (Şahin \& Cemaloğlu, 2015). A collaborative culture makes schools more attractive to teachers and tends to result in lower staff attrition rates (Berry et al., 2009). A strong collective collaborative culture has been linked to higher student achievement (Gruenert, 2005; Lee \& Louis, 2019), regardless of the varying levels of collaborative efforts from each teacher (Ronfeldt et al., 2015). In fact, collaboration has shown to be more effective to turning around low performance in schools than removing and replacing ineffective teachers (Berry et al., 2008). This has some schools in constant pursuit of the strongest collaborative culture they can achieve.

Many factors need to be considered to create a strong collaborative culture. The most prevalent factor in research is to allot proper time for PLC's to interact (Balfanz, 2011; Berry et al., 2008; Dorman, 2012; Gajda \& Koliba, 2008; Goddard, Goddard, Sook Kim, \& Miller, 2015; Goddard \& Kim, 2018; Kallemeyn, 2014; McIntosh \& White, 2006; Slick, 2002). Many schools take it a step further and embed a common planning time into their schedule throughout the year (Hazel et al, 2014; Jacobson, 2010; Kallemeyn, 2014; Parrish et al., 2011; Şahin \& Cemaloğlu, 2015). Teachers report that when given ample opportunity to interact with their colleagues, they are more apt to actively participate (Johnston \& Tsai, 2018). This means that the session duration needs to be long enough, and collaboration should also be sustained continuously throughout the year (Akiba, 2012; Goddard et al., 2015; Goddard \& Kim, 2018; Kallemeyn, 
2014; McIntosh \& White, 2006; Nelson et al., 2007). The increasing demands teachers face make efficiently structuring that collaboration crucial for maximizing their depleted availability.

To create high quality teacher collaboration, creating space and time are simply not enough (Gajda \& Koliba, 2008). Before schools can grasp a better understanding of how to structure PLC's, they must first be intentional with the purpose and objectives of the collaboration. Through this, the entire staff can work under a recommended shared vision (Nelson et al., 2007; Parrish et al., 2011; Roybal et al., 2014). School leaders should implement a strategic PLC plan and structure that aligns with this mission (Akiba, 2012; McIntosh \& White, 2006). When schools have common frameworks that guide their collaboration strategies and goals, they have higher quality experiences than those who collaborate without a framework or fail to collaborate at all (Gajda \& Koliba, 2008; Youngs, Holdgreve-Resendez, \& Qian, 2011). This can be critical to a school's IPC as well. Jacobson (2010) recommended schools build IPC with $\mathrm{PLC}^{\prime} \mathrm{s}$ through coordinating the teams and their responsibilities in a manner that builds upon previous progress and does not haphazardly unfold or counteract the work of others. This will help schools to follow through on initiatives more thoroughly.

\section{Collaboration Focus and Instructional Program Coherence}

The focus of collaboration is as important as having an optimal environment for it to occur (Means, 2010). While professional development has historically been expected to center around content, it has since deviated to focus on process, practice, and climate (Nelson et al., 2007). Most collaboration objectives can be classified within one or a combination of the IPC domains: curriculum, instruction, assessment, and academic climate (Newmann et al., 2001).

A fundamental focus of collaboration is on the vertical alignment of curriculum and standards with focus on student success (Cooney \& Bottoms, 2003; Gilmer, 2010; Ronfeldt et 
al., 2015; Parrish et al., 2011; Schlosser, 2015; 2015; Sekulich, 2019). Curriculum mapping should be practiced through ongoing communication between grade levels and school buildings (Kowal, 2002; Morgan \& Hertzog, 2001; Sekulich, 2019). Mapping gives teachers a better breadth of understanding of the entire learning pathway of students from kindergarten through high school and better define their place within it (Gilmer, 2010; Kowal, 2002). Knowing the mastery content going into and upon completion of grade levels allows teachers to avoid overlap and rectify gaps in the scope and sequence of learning (Crawford, 2011; Parrish et al., 2011, Smith, 2007). Unfortunately, curriculum alignment is difficult to achieve across buildings due to logistic and funding challenges (Parrish et al. 2011).

Instructional practice and alignment to curriculum collaboration is found by teachers to be more extensive and helpful than other areas of focus (Ronfeldt et al., 2015; Schlosser, 2015; Smith, 2006a). Johnston and Tsai (2018) found that when the volume of feedback teachers received from their peers increased, so did their positive perception that the feedback was helpful in improving their instruction. Teacher collaboration has been proven to improve teaching quality (Ismail et al., 2018) as teachers become less isolated in their instructional methodology (Gilmer, 2010). Strong departmental and vertical teams increase opportunities for dissemination of practices throughout the teaching staff (Akiba, 2012; Corcoran \& Silander, 2009). These high functioning teams use collegial feedback to make purposeful adjustments to improve the quality and merit of their practice to increase student learning (Donohoo \& Katz, 2019; Gajda \& Koliba, 2008). Educators do this by working together to experiment through the employment of multiple student-centered approaches. They then follow the evidence to continue those strategies found to be effective (Corcoran \& Silander, 2009; Roybal et al., 2014) and also align with required state and local curriculum frameworks (Schlosser, 2015, Nelson et al., 2007). 
It has been shown that collaboration focused on instruction builds the instructional capacity of individual teachers and the overall staff (Crawford, 2011). These teachers tend to keep students more engaged with interesting classroom strategies (Butts \& Cruziero, 2005). Teachers engaged in collaboration were more likely to employ differentiated instruction and pedagogy that was more challenging and thoughtful (Goddard \& Kim, 2018). Overall, teachers "can increase instructional capacity by developing strategies, sharing strategies, and discussing the pros and cons of implementation of the various strategies at different grade levels, all for the purpose of improving student learning" (p. 4, Crawford, 2011).

Schools must continually assess their school improvement and provide tangible evidence of its effectiveness (Donahoo \& Katz, 2019; Gajda \& Koliba, 2008). Collecting comparative evidence requires the methods of measurement to be consistent by utilizing student data through common assessments (Jacobson, 2010). Some schools use standardized state tests as a guide to create common assessments (Nelson et al., 2007) because it provides a common measurement system, academic language, and outcomes that can be measured against other schools (Parrish et al., 2011). Collaboration around creating common assessments however is often more predictive of school and teacher performance and student achievement than any other collaborative focuses (Ronfeldt et al., 2015). Therefore, teacher teams should collaboratively design, and peer review assessments based on desired learning results and use student work from these measures to identify student misconceptions (McTighe \& Emberger, 2006; Ronfeldt et al., 2015; Schlosser, 2015). The data provide feedback for staff to intervene with struggling students (Gilmer, 2010; Nelson et al., 2007), but also to evaluate the effectiveness of their own teaching. 


\section{Vertical Collaboration and the Transition to High School}

The educational progression of a student is a continuous process that builds upon previous years where it is assumed most students are ready for the learning that should take place at their grade level (Masters, 2005). However, this progression is marred by discontinuities. With that in mind, "dropout reduction is a vertical project" (Henderson, 2000, p. 2). By virtue, this implies that combating the discontinuities that lead to dropping out of school are also a vertical project. For sustainable improvement, the educational system needs to shift from a within-school to between-school view (Prenger, Poortman, \& Handelzalts, 2019). Transitions from mid-level feeder schools to high school need to be studied and improved so all teachers are on the same page when managing the academic, social, and organizational factors that create such a unique and often difficult experience for students (Nelson et al., 2007; Smith et al., 2008).

Schulz and Geithner (2010) stated, "Although the awareness of the requirement of change within the school systems has been high, little is known about how sustainable intervention and innovation can be brought about within schools through collective exchange and learning beyond organizational borders" (pp. 69-70). It is widely suggested in research that communication and collaboration between mid-level feeder school and high school teachers needs to be increased and improved (Akos \& Galassi, 2004; Chapman \& Sawyer, 2001; Dorman, 2012; Egodawatte et al., 2011; Fulk, 2003; Hazel et al., 2014; Kowal, 2002; Mizelle, 2005; Morgan \& Hertzog, 2001; Neild et al., 2007; Oakes, A., 2009; Parrish et al., 2011; Schlosser, 2015; Smith, 2006a; Smith et al., 2008). The collaborative focuses for teachers that contribute to successful transitions for students mirror those of small learning communities, vertical teams, and grade-level teams (Nelson et al., 2007). Aligning curriculum (Akos \& Galassi, 2004; Kowal, 2002; Masters, 2005; Mizelle \& Irvin, 2000; Morgan \& Hertzog, 2001; Parrish et al., 
2011; Schlosser, 2015; Smith, 2007; Suh \& Seshaiyer, 2015), improving instruction (Fulk, 2003;

Kowal, 2002; Parrish et al., 2011; Smith, 2006a), and communicating academic expectations (Akos \& Galassi, 2004; Cooney \& Bottoms, 2003; Mizelle \& Irvin, 2000; Smith, 2007, Schlosser, 2015) across the divide remained consistent with other research in collaboration. Even with recommended collaboration in research, less than half of high school teachers have ever met with their feeder mid-level feeder school teachers to discuss such topics (Cooney \& Bottoms, 2003).

The responsibility to ensure all students transition successfully into high school is not solely placed on one institutional level but is mutual and shared throughout (Hazel et al., 2014; Mizelle \& Irvin, 2000). When these ongoing intra-school efforts are made, consistency is formed by the way of common terminology, instruction, learning environments, assessments, and academic expectations (Henderson, 2000) that often lead to higher student achievement.

\section{Research Design and Analysis}

To address the research questions, a quantitative study was conducted (Creswell, 2014; Merriam \& Tisdell, 2016). The following section summarizes the study by outlining the setting, participants, data collection methods and tools, and the means of data analysis.

\section{Setting}

The setting of this study took place in public high schools in the state of Missouri. In the 2019-2020 school year, statistics showed that the total number of students enrolled Missouri high schools in the fall was 267,527 (DESE, 2020). The diversity breakdown at the beginning of the 2019-2020 school year for the state of Missouri was as follows: 70.7\% of students were White; 15.7\% were Black; $6.7 \%$ were Hispanic; $4.3 \%$ were Multi-racial; $2.1 \%$ were Asian; and the remaining 0.5\% identified as American Indian or Pacific Islander (DESE, 2020). Students with 
limited English Language proficiency made up 3.9\% of the student population. A little over half of the students in Missouri qualified for free or reduced lunch in the 2019-2020 school year (DESE, 2020). In the 2018-2019 school year, Missouri was the 27th ranked state with an average composite ACT score of 20.8, which was slightly above the national average of 20.7 (Statista, n.d.). Missouri's 4-year graduation rate in 2019 was 89.58\% (DESE, 2020), meaning an estimated 27,783 high school students in the state would not graduate within four years.

\section{Participants}

The participants in this study were all the principals in Missouri public high schools whose structure houses grades 9 through 12 within their building and have separate geographically located mid-level feeder schools. To find the specific principals that fit the required criteria, the state school directory from DESE (2020) was utilized. A filtration system determined which high schools consisted of the appropriate grades. From that list, the researcher went through and removed all non-traditional high schools, such as technical schools and career academies. Finally, the remaining schools were analyzed to see if they had mid-level feeder schools in separate geographic locations. Overall, 312 high schools qualified to create the population for this study and the principals of those schools were invited to participate. The entire population was invited to participate in the survey.

An additional requirement was added to the study that required participating principals to have over one year of administrative experience in their building. This was done for two reasons. The first is new principals may not yet have an accurate portrayal of the IPC of their school. The second was that due to the Covid-19 pandemic in 2020 , this study required data from the 2018-2019 school year. To omit first year principals, section one in the survey 
consisted of a qualifying question to find the principal's years of experience. If it was a year or less, they were redirected to the end of the survey and were void from the participant pool.

\section{Data Collection}

Electronic Survey: A survey was electronically sent to all high school principals using Qualtrics. This consisted 35 questions (27 ordinal, 7 numerical, and 1 short answer) broken down into three sections.

- Section 1: Qualifying Questions. This section consisted of informed consent and a question to eliminate participants with one year of experience or less within that school.

- Section 2: School Data. This section gathered all freshmen data not obtainable by the researcher through DESE. It also included a request for contact information for someone within the district with this data in case it was not easily accessible by the principal.

- Section 3: Instructional Program Coherence. This section measured the IPC focus of high school collaboration. It was adapted from Newmann et al.'s (2001) Likert-scale survey by incorporating different IPC components: curriculum, instruction, assessment, academic climate, and vertical collaboration. Subscales of the five IPC components were created and averaged for the predictor variables in the study.

The survey was conducted from December 1, 2020, to February 28, 2021. Respondents received email reminders to complete the survey on average of every two weeks. The duration was extended in attempt to gain the amount of responses that validly represent the population (Field, 2018; Fink, 2017). At the halfway point, a paper survey identical to the electronic survey was mailed to the principals who had not yet responded to increase the response rate (see Appendix). A self-addressed stamped envelope was included for their return. Phone message reminders were left to increase involvement before the survey deadline. 
DESE Records: Some dependent data were obtained by the researcher through online DESE records (DESE, 2020). These records included graduation rates, dropout rates, freshman course failure rates, out-of-school suspension rates, attendance rates, and EOC scores. Free-andreduced lunch rates and enrollment data from DESE were used in this study.

Ethical consideration. Principals agreed to an informed consent form before any form of participation. The researcher followed Seidman's (2013) Eight Major Parts of Informed Consent as well as the University of Missouri Institutional Review Board standards. Participants had full transparency and withdrawal rights throughout. The questionnaire from Fink (2017, p. 24) provided ethical technological guidelines for this study. By using school emails provided by DESE, the researcher is assured of their authentication. Qualtrics was used through the University of Missouri to ensure secure data storage and encryption.

\section{Data Analysis}

Statistical analyses were conducted for all three research questions using Statistical Package for the Social Sciences (SPSS). For RQ1, the dependent variable descriptive statistics were found by finding the means, maximums, minimums, and standard deviations of the output data from DESE and the items found from section two of the survey. The subscale score for each IPC component was be found by taking the mean scores of the questions that apply to each. This showed to what extent schools focus on each. The overall IPC was calculated by taking the mean of the component subscale scores.

To answer RQ2, bivariate correlations were conducted using the overall IPC score and the curriculum, instruction, assessment, academic climate, and vertical collaboration subscale scores as predictor variables. The response variables were positive student achievement measures: graduation rate, EOC scores, attendance rate, and average freshman GPA. A linear 
regression was run to see how well the overall IPC predicted each student achievement measure when controlling for socioeconomic status. This process was repeated for RQ3. However, the response variables for each were negative student achievement measures: dropout rate, course failure rate, and the freshman OSS rate. Socioeconomic status was controlled in the linear regressions for both RQ2 and RQ3 using the 2018-2019 free-and-reduced lunch data for each school obtained from DESE (DESE, 2020).

\section{Findings}

Findings for this study were produced from online surveys and data collected from DESE (2020). The population of this study consisted of 312 public high school principals from the state of Missouri with grades 9-12 and mid-level feeder schools in separate geographical locations. The survey garnered 43 total responses, but three principals declined to consent and eight were void from the study for being in an administrative position within their building for one year or less. The remaining 32 qualified participants provided useable data, which represents approximately $10.3 \%$ of the overall population. Of those 32 participants, six failed to provided part or all of their freshmen data. The descriptive statistics from the participating schools provide the dependent and control variables for the study and are shown in Table 1. Descriptive statistics, bivariate correlation, and linear regression were conducted to answer the three research questions driving this study. The following examines those results.

\section{RQ1: As perceived by high school principals, to what extent is IPC utilized in Missouri} secondary schools?

To answer RQ1, mean scores were taken for each IPC component using their specified items from section three of the survey. The overall IPC scores were then found by taking the mean score of the five subscale means found. The scale was adapted from Newmann et al.'s 
(2001) IPC 4-point Likert scale as follows: $1=$ not at all, $2=$ some or needs improvement, $3=$ most or emerging, and $4=$ to great extent or excelling. The results are shown in Table 2.

Principals' average perception was that their building had emerging overall IPC at 3.07. The highest component was academic climate, followed by curriculum, instruction, and assessment. These four components had a score of emerging, with a range of .14 between the lowest (3.09) to the highest (3.23). The lowest overall, vertical collaboration was the only component perceived as needing improvement with a score of 2.70. This was .39 less than the next lowest composite score and dragged the overall IPC mean below the other four means. The vertical collaboration component also had the highest overall spread (2.60) and average spread (.54), among all the components. An assumption can be made that while overall most schools value vertical collaboration somewhat less than the other four components, the disparity between the scores and the wider spread also show that a few schools prioritize vertical collaboration much less. This is reaffirmed with the score of 2.70 being much closer to the emerging category than it is to having no vertical collaboration at all.

RQ2: What is the relationship between IPC (as perceived by high school principals) among mid-level feeder school and high school teachers and student achievement as measured by: graduation rate, EOC scores, average freshman GPA, and attendance rate?

The results of the bivariate correlation to answer RQ2 are found in Table 3. The overall IPC had positive correlation with the graduation rate and EOC Algebra 1 scores and were extremely close to being statistically significant, with $p=.056$ and $p=0.80$ respectively. The overall IPC showed virtually no correlation and was not close to being statistically significant any of the other outcomes: EOC English 2, EOC Biology, average freshman GPA, and freshmen attendance rate. For the outcome variables EOC English 2, EOC Biology, and freshmen 
attendance rate, all of the IPC components showed no correlation nor significance. However, all the other dependent variables had statistics that showed associative patterns.

The graduation rate had a positive association with the instruction component (.378) and the curriculum component (.384). Both were statistically significant at a 0.05 level with $p=.033$ and $p=.030$ respectively. In fact, the graduation rate had a near statistically significant positive association with assessment and academic climate as well. The academic climate showed a positive correlation (.435) with the Algebra 1 EOC scores at a statistically significant level with $p=.013$. Vertical collaboration showed a positive correlation with the average freshman GPA (.542) and was the only correlation significant at the 0.01 level with $p=.004$. Vertical collaboration showed the least correlation and no significance with any other outcome variable.

A linear regression was performed to see how much the overall IPC would predict the positive student achievement outcomes and is shown in Table 4. This was done with controlling for socioeconomic status using the free and reduced lunch data from DESE (2020). The only two outcome variables that were close to being statistically significant when using overall IPC to predict their variance were the graduation rate with $p=.062$, and EOC Algebra 1 with $p=.096$. Results show that $9.4 \%$ of the graduation rate variance is predicted by overall IPC. Overall IPC also predicted approximately $8.4 \%$ of the EOC Algebra 1 score variance. The remaining four dependent variables showed virtually no predictability based on overall IPC.

RQ3: What is the relationship between IPC (as perceived by high school principals) among mid-level feeder school and high school teachers and student achievement as measured by: dropout rate, freshmen course failure rate, and freshmen OSS rate?

The results of the bivariate correlation to answer RQ3 are found in Table 5. Unexpectedly, all of the IPC components and the overall IPC showed a positive association with 
both freshmen course failure and OSS rates. The only instance where this was not the case was with vertical collaboration and the freshmen course failure rate, although that was virtually no correlation at -.025 . This anomaly could be attributed to the extremely low response rate in combination with the specificity of the measurement. Many of these data values were mere fractions of percentage points away from each other. It is also shown in the fact that none of them were close to being statistically significant.

Academic climate showed a significant negative correlation with the dropout rate (-.351) at the 0.05 level with $p=.049$. In fact, instruction, curriculum, assessment, and overall IPC all showed a negative correlation with the dropout rate that was close to being statistically significant. Vertical collaboration was the only measure that showed virtually no correlation or significance with the dropout rate. In further investigation, removing the vertical collaboration component from the data analysis, changed the correlation between the overall IPC and the dropout rate from -.286 to -.336 . The significance also changed from $p=.113$ to $p=.060$.

A linear regression was performed to see how much the overall IPC would predict the negative student achievement outcomes and is shown in Table 6. This was done with controlling for socioeconomic status using the free and reduced lunch data from DESE (2020). The only outcome variable that was relatively close to being statistically significant when using overall IPC to predict its variance was the dropout rate with $p=.131$. Overall IPC predicted approximately $6.3 \%$ of the dropout rate's variance in this instance.

\section{Discussion and Recommendations}

The extent to which Missouri high school principals utilize IPC is relatively stable and positive across all domains but vertical collaboration. This was expected as research has overwhelmingly recommended to focus on curriculum, instruction, and assessment in 
collaboration because of the positive student and teacher gains it produces. Curriculum and instruction have historically been the focus of collaboration (Cooney \& Bottoms, 2003; Gilmer, 2010; Ronfeldt et al., 2015; Parrish et al., 2011; Schlosser, 2015) which makes it understandable why principals perceive it so positively in their buildings.

The academic climate was the most positive component perceived by principals. This goes against much of the research that shows the academic climate disparities between levels (Cooney \& Bottoms, 2003; Emmet \& McGee, 2012; Neild et al., 2007; Neild, 2009). The other elements of Newmann et al's (2001) concept of IPC could account for this paradox. Intentionally held professional development is usually sustainable, whole staff oriented, and requires substantial resources. These are all items measured in the academic climate portion of IPC that may cause a misperception to the actual academic climate found in the classroom. One way to grow the in-class academic climate may result in collaborating around common assessments. Ronfeldt et al. (2015) showed collaboration around common assessment creation is often more predictive of student achievement than any other collaborative focuses (Ronfeldt et al., 2015). Yet, assessment was the second lowest scored IPC component in this study.

Vertical collaboration was by far the lowest priority of IPC perceived by principals and had the least correlation with all the outcome variables except one. This coincides with Bergman et al's (1998) study that illustrated vertical collaboration for curriculum alignment had no correlation with student achievement on the tenth grade Texas standardized test. However, when dealing with the freshmen, vertical collaboration had the strongest and most positive correlation with academic success as measured by average freshman GPA. A heightened average freshman GPA means that less students will end the year off track for graduation because they are 
delinquent on credit accrual, which leads to a higher likelihood of dropping out (Neild, 2009; Mizelle \& Irvin, 2000; Smith et al., 2008; Uvaas \& McKevitt, 2013).

High schools are taking great measures to combat the struggles for students brought on by the transition from mid-level feeder schools. These measures include creating small learning communities (Balfanz, 2011; Bornsheuer et al., 2018; Cauley \& Jovanovich, 2006; Corcoran \& Silander, 2009; Dedmond et al., 2006), tutoring and mentoring programs (Fulk, 2003; Kennelly \& Monrad, 2007; Letrello \& Miles, 2003; Mizelle, 2005; Parrish et al., 2011; Smith, et al., 2008), early risk identification and intervention (Balfanz, 2011; Balfanz et al., 2007), and a summer transition program or freshman preparatory course (Hazel et al., 2014; Horwitz \& Snipes, 2008; Kennelly \& Monrad, 2007; Parrish et al., 2011; Smith, et al., 2008; Vera et al., 2016). These are usually the focus of collaboration between mid-level feeder and high schools. The more reform practices implemented by schools that specifically target the freshman transition results in greater student learning (Morgan \& Hertzog, 1998; Neild, 2009). The recommendation this study implies is that districts should increase the vertical collaboration between high schools and their feeder schools and incorporate focus on the curriculum, instruction, and assessment components to see if that will result in further academic achievement in different areas. While principals perceive the IPC components to be strong within their buildings, the academic disconnect with their mid-level feeder schools still remain.

\section{Limitations}

The most glaring limitation to this study is the low response rate. This can be attributed to many factors. In the spring of 2020, the Covid-19 pandemic hit the United States, which forced schools to face unforeseen challenges. This carried over into the 2020-2021 school year where high school principals' focus was adapting their school structures and protocols to conduct 
school in the safest manner possible. The researcher received numerous emails where the sender apologized for their inability to participate in the study because of the unprecedented situation they were facing at their school due to the pandemic.

Also contributing to the low response rate was the low prioritization by principals of this topic as being one in need of focus. The lack of necessity can result from many factors. Schools operate under a district structure that has a curriculum position that is often attributed with the responsibility of ensuring learning continuity between building levels. Districts operate with an understanding that the state curriculum is aligned accordingly so that all content is covered. In addition, many high schools are at different points of implementing programs to ease the transition for incoming freshmen and may have seen some positive results or the results are pending. These programs often overlook the role IPC can play in the disruption, and therefore, do not incorporate the necessary vertical collaboration. Finally, very little research exists examining the specific dynamics of the relationship between mid-level feeder school teachers and high school teachers when pertaining to academics. With this being uncharted in research it is unknown to principals and therefore not seen as a priority. Further research could expose a need for their future participation.

Another limitation to this study is that it is a singular cross-sectional study as opposed to a longitudinal one. Due to the Covid-19 pandemic, the 2019-2020 End-of-Course (EOC) exams were cancelled by DESE and many school districts altered their grading scale to compensate. To respond, this study had participants pull student data from the 2018-2019 school year and required only principals who have been in their school's administrative cabinet for more than a year. Another reason is that collaborative programs tend to have better student achievement results the longer they are sustained (Jacobson, 2010). In a longitudinal study, the trends of 
student achievement growth as programs progress could be examined. Schools that recently implemented freshman transition interventions may not have seen the results yet and that could alter the outcome of the analysis of the collaborative efforts relationship to student achievement.

The participants' subjective interpretation of the definition of intentional collaboration is another limitation. While it is defined by the researcher, principals may define it differently based on the context of their setting and staff. Also, collaboration may occur outside of professional development or common planning time that was not counted by the principal in this study yet may influence student success. In the reverse effect, principals may embellish the amount of collaboration or IPC level to obtain a more socially desirable impression of their school and their work. A further limitation on this study is the researcher's positional bias that school districts are not addressing the academic continuity between mid-level feeder school teachers and high school teachers, which was addressed throughout every portion of the study.

\section{Future Research}

The idea of vertical collaboration to increase IPC across the transition to high school is a relatively new idea in educational research. One purpose of this study was to start the discussion on this focus. Therefore, further research is warranted as this study overall was inconclusive to the role vertical collaboration plays in building IPC between high school and feeder school teachers as well as its influence on student achievement.

One recommendation is to do a longitudinal study that involves teachers across the transition. The perception of principals may be very different from those that work directly with the students. This could be done quantitatively with the same survey or a similarly adapted one or qualitatively to give the study more depth and address what schools are doing to achieve IPC between buildings. One way to make it longitudinal is to find a school implementing a vertical 
collaboration plan between levels, collect student achievement data over a few years and compare it to previous data. Another possibility is to find a high achieving school that deploys a collaboration program that has shown good results and do a case study on them. This may notify other districts that there is value in this topic's pursuit. You could make it a multi-case comparative study with a school or schools that do not collaborate vertically throughout the district. Regardless of the study, the continued academic disconnect between middle school and high school warrants further research. 


\section{References}

Akiba, M. (2012). Professional learning activities in context: A statewide survey of middle school mathematics teachers. Education Policy Analysis Archives, 20(14). doi:10.14507/epaa.v20n14.2012

Akos, Patrick., \& Galassi, J. P. (2004). Middle and high school transitions as viewed by students, parents, and teachers. Professional School Counseling, 7(4), 212-221.

Allensworth, E. M., \& Easton, J. Q. (2007). What matters for staying on-track and graduating in Chicago public high schools: A close look at course grades, failures, and attendance in the freshman year. Research Report. Consortium on Chicago School Research. Retrieved from https://files.eric.ed.gov/fulltext/ED498350.pdf

Alspaugh, J. W. (1998). Achievement loss associated with the transition to middle school and high school. Journal of Educational Research, 92(1), 20.

Anderman, E. M., \& Midgley, C. (2004). Changes in self-reported academic cheating across the transition from middle school to high school. Contemporary Educational Psychology, 29(4), 499-517.

Balfanz, R. (2011). Back on track to graduate. Educational Leadership, 68(7), 54-58.

Balfanz, R., Herzog, L., \& Mac Iver, D. J. (2007). Preventing student disengagement and keeping students on the graduation path in urban middle-grades schools: Early identification and effective interventions. Educational Psychologist, 42(4), 223-235. doi:10.1080/00461520701621079

Benner, A. D. (2011). The transition to high school: current knowledge, future directions. Educational Psychology Review, 23(3), 299. doi:10.1007/s10648-011-9152-0 
Benner, A. D., Boyle, A., \& Bakhtiari, F. (2017). Understanding students' transtion to high school: Demographic variation and the role of supportive relationships. Journal of Youth \& Adolescence, 46(10), 2129-2142. https://doi-org. /10.1007/s10964-017-0716-2

Benner, A. D. \& Graham, S. (2009), The transition to high school as a developmental process among multiethnic urban youth. Child Development, 80, 356-376. doi:10.1111/j.14678624.2009.01265.x

Benner, A. D., \& Wang, Y. (2014). Demographic marginalization, social integration, and adolescents' educational success. Journal of Youth \& Adolescence, 43(10), 1611-1627. doi:10.1007/s10964-014-0151-6

Bergman, D., Calzada, L., LaPointe, N., Lee, A., \& Sullivan, L. (1998). Vertical alignment and collaboration (Doctoral dissertation, Texas A\&M University, Corpus Christi/Kingsville). Retrieved from https://files.eric.ed.gov/fulltext/ED421472.pdf

Berry, B., Daughtrey, A., \& Wieder, A. (2009, December). Collaboration: Closing the effective teaching gap. Center for Teaching Quality.

Bornsheuer, J. N., Polonyi, M. A., Andrews, M., Fore, B., \& Onwuegbuzie, A. J. (2018). The relationship between ninth-grade retention and on-time graduation in a Southeast Texas high school. The Journal of At-Risk Issues, 16(2), 9.

Brown, L. C. \& Seeley, C. L. (2010). Transitions from middle school to high school: crossing the bridge. Mathematics Teaching in the Middle School, 15(6), 354-358.

Butts, M. J., \& Cruzeiro, P. A. (2005). Student perceptions of factors leading to an effective transition from eight to nine grade. American Secondary Education, 34(1), 70. 
Casillas, A., Robbins, S., Allen, J., Kuo, Y.-L., Hanson, M. A., \& Schmeiser, C. (2012). Predicting early academic failure in high school from prior academic achievement, psychosocial characteristics, and behavior. Journal of Educational Psychology, 104(2), 407-420. doi:10.1037/a0027180

Cauley, K. M., \& Jovanovich, D. (2006). Developing an effective transition program for students entering middle school or high school. Clearing House, 80(1), 15-25. doi:10.3200/TCHS.80.1.15-25

Chapman, C., Laird, J., Ifill, N., \& Kewal Ramani, A. (2011). Trends in High School Dropout and Completion Rates in the United States: 1972-2009. Compendium Report. NCES 2012-006. National Center for Education Statistics. Retrieved from http://nces.ed.gov/pubsearch.

Chapman, M. V., \& Sawyer, J. S. (2001). Bridging the gap for students at risk of school failure: A social work-initiated middle to high school transition program. Children \& Schools, 23(4), 235. doi:10.1093/cs/23.4.235

Cohen, J. S., \& Smerdon, B. A. (2009). Tightening the dropout tourniquet: Easing the transition from middle to high school. Preventing School Failure, 53(3), 177. doi:10.3200/PSFL.53.3.177-184

Cooney, S., \& Bottoms, G. (2003). Middle grades to high school: Mending a weak link. (Research Brief No. 143). Department of Education, Southern Regional Education Board. Retrieved from https://www.sreb.org/programs/hstw/publications/briefs/Middle_Grades_To_HS.pdf 
Cooper, R., \& Liou, D. D. (2007). The structure and culture of information pathways: Rethinking opportunity to learn in urban high schools during the ninth-grade transition. High School Journal, 91(1), 43. doi:10.1353/hsj.2007.0020

Corcoran, T., \& Silander, M. (2009). Instruction in high schools: The evidence and the challenge. The Future of Children, 19(1), 157-183. doi:10.1353/foc.0.0026

Crawford, S. T. (2011). Increasing instructional capacity through vertical teaming. Retrieved from https://rb.gy/vqi6wl

Creswell, J. W. (2014). Research design: Qualitative, quantitative, and mixed methods approaches (4th ed.). Thousand Oaks, CA: Sage.

Dedmond, R., Brown, R. D., \& LaFauci, J. M. (2006). Freshman transition programs: Long-term and comprehensive. Principal's Research Review, 1(4), 1-8.

DeLamar, S., \& Brown, C. G. (2016). Supporting transition of at-risk students through a freshman orientation model. Journal of At-Risk Issues, 19(2), 32-39.

Donohoo, J., \& Katz, S. (2019). What drives collective efficacy? Effective teams that believe they can make a difference create the conditions to get better in four key ways. Educational Leadership, 76(9), 24.

Dorman, B. (2012). The supported teen: Transitioning to high school. Leadership, 41(5), 22-23. Egodawatte, G., McDougall, D., \& Stoilescu, D. (2011). The effects of teacher collaboration in Grade 9 Applied Mathematics. Educational Research for Policy and Practice, 10(3), 189-209. doi:10.1007/s10671-011-9104-y

Ellerbrock, C. R., \& Kiefer, S. M. (2014). Supporting young adolescents' middle-to-high-school transition by creating a ninth-grade community of care: Implications for middle grades educators. Middle School Journal, 45(3), 3-10. doi:10.1080/00940771.2014.11461886 
Emmett, J., \& McGee, D. (2012). A farewell to freshmen. Clearing House, 85(2), 74-79. doi:10.1080/00098655.2011.619592

Field, A. (2018). Discovering statistics using IBM SPSS statistics (5th ed.). Thousand Oaks, CA: Sage.

Fink, A. (2017). How to conduct surveys: A step-by-step guide (6th ed). Thousand Oaks, CA: Sage.

Fulk, B. M. (2003). Concerns about ninth-grade students' poor academic performance: One school's action plan. American Secondary Education, 31(2), 8-26

Gajda, R., \& Koliba, C. J. (2008). Evaluating and improving the quality of teacher collaboration: A field-tested framework for secondary school leaders. NASSP Bulletin, 92(2), 133-153. doi:10.1177/0192636508320990

Galla, B. M., Tsukayama, E., Park, D., Yu, A., \& Duckworth, A. L. (2020). The mindful adolescent: Developmental changes in nonreactivity to inner experiences and its association with emotional well-being. Developmental Psychology, 56(2), 350-363. doi:10.1037/dev0000877

Gilmer, P. J. (2010). Vertical teaming: K-12 teachers engaged in scientific research in rural settings. The Rural Educator, 31(3), 1-6.

Goddard, R., Goddard, Y., Sook Kim, E., \& Miller, R. (2015). A theoretical and empirical analysis of the roles of instructional leadership, teacher collaboration, and collective efficacy beliefs in support of student learning. American Journal of Education, 121(4), 501-530. doi:10.1086/681925 
Goddard, Y., \& Kim, M. (2018). Examining connections between teacher perceptions of collaboration, differentiated instruction, and teacher efficacy. Teachers College Record, 120(01301), 1-24.

Gruenert, S. (2005). Correlations of collaborative school cultures with student achievement. NASSP Bulletin, 89(645), 43-55. doi:10.1177/019263650508964504

Hazel, C. E., Pfaff, K., Albanes, J., \& Gallagher, J. (2014). Multi-level consultation with an urban school district to promote 9th grade supports for on-time graduation. Psychology in the Schools, 51(4), 395-420. doi:10.1002/pits.21752

Henderson, C. (2000). Vertical teams yield vertical achievement in Southeastern Texas school district. Education in Practice, 9, 1-4.

Herlihy, C., \& Kennelly, L. (2007, May). State and district support for successful transitions into high school. Washington, DC: National High School Center

Horwitz, A., \& Snipes, J., (2008). Research brief: Supporting successful transitions to high school. Washington, DC: The Council of Greater City Schools

Huerta, J., J., Watt, K. M., \& Butcher, J. T. (2013). Examining Advancement Via Individual Determination (AVID) and its impact on middle school rigor and student preparedness. American Secondary Education, 41(2), 24-37.

Ismail, S. N., Kanesan, A. G., \& Muhammad, F. (2018). Teacher collaboration as a mediator for strategic leadership and teaching quality. International Journal of Instruction, 11(4), 485-498. doi:10.12973/iji.2018.11430a

Jacobson, D. (2010). Coherent instructional improvement and PLCs: Is it possible to do both? The Phi Delta Kappan, 91(6), 38-45. 
Johnston, W., \& Tsai, T. (2018). The Prevalence of Collaboration Among American Teachers: National Findings from the American Teacher Panel. (RAND Corporation Research Report RR-2217-BMGF). doi:10.7249/RR2217

Kallemeyn, L. M. (2014). School-level organizational routines for learning: Supporting data use. Journal of Educational Administration, 52(4), 529-548. doi:10.1108/JEA-02-2013-0025

Kennelly, L., \& Monrad, M. (2007). Easing the transition to high school: Research and best practices designed to support high school learning. National High School Center. Retrieved from https://files.eric.ed.gov/fulltext/ED501073.pdf

Kowal, P. H. (2002). Vertical teaming: Making connections across levels. Middle Ground, 6(1), 20-22.

Langenkamp, A. G. (2009). Academic vulnerability and resilience during the transition to high school: The role of social relationships and district context. Sociology of Education, 83(1), 1-19. https://doi.org./10.1177/0038040709356563

Lee, M., \& Louis, K. S. (2019). Mapping a strong school culture and linking it to sustainable school improvement. Teaching and Teacher Education, 81, 84-96. doi:10.1016/j.tate.2019.02.001

Letrello, T. M. \& Miles, D. D. (2003). The transition from middle school to high school: Students with and without learning disabilities share their perceptions. The Clearing House, 76(4), 212.

Lovelace, M. D., Reschly, A. L., \& Appleton, J. J. (2017). Beyond school records: the value of cognitive and affective engagement in predicting dropout and on-time graduation. Professional School Counseling, 21(1). doi:10.5330/1096-2409-21.1.70 
Madda, C. L., Halverson, R., \& Gomez, L. M. (2007). Exploring coherence as an organizational resource for carrying out reform initiatives. Teachers College Record, 109(8), 1957-1979.

Masters, J. (2005). Continuity and growth: Key considerations in educational improvement and accountability. (AIRTV Seminar Series Paper No. 141). Retrieved from http://research.acer.edu.au/cgi/viewcontent.cgi?article=1009\&context=monitoring_learni ng

McCallumore, K. M., \& Sparapani, E. F. (2010). The importance of the ninth grade on high school graduation rates and student success. Education Digest, 76(2), 60.

McIntosh, J., \& White, S. H. (2006). Building for freshman success: high schools working as professional learning communities. American Secondary Education, 34(2), 40.

McKee M. T., \& Caldarella P. (2016). Middle school predictors of high school performance: A case study of dropout risk indicators. Education, 136(4), 515-529.

McTighe, J., \& Emberger, M. (2006). Teamwork on assessments creates powerful professional development. Journal of Staff Development, 27(1), 38-44.

Means, B. (2010). Technology and education change: Focus on student learning. Journal of Research on Technology in Education, 42(3), 285-307. doi:10.1080/15391523.2010.10782552

Merriam, S. B., \& Tisdell, E. J. (2015). Qualitative research: A guide to design and implementation (4th ed.). San Francisco, CA: Jossey-Bass.

Missouri Department of Elementary and Secondary Education (DESE). (2020). Missouri comprehensive data system: Missouri school statistics. Retrieved from: https://apps.dese.mo.gov/MCDS/Home.aspx

Mizelle, N. B. (2005). Moving out of middle school. Educational Leadership, 62(7), 56-60. 
Mizelle, N. B., \& Irvin, J. L. (2000). Transition from middle school into high school. Middle School Journal, 31(5), 57-61. doi:10.1080/00940771.2000.11494654

Moolenaar, N. M., Sleegers, P. J. C., \& Daly, A. J. (2012). Teaming up: Linking collaboration networks, collective efficacy, and student achievement. Teaching and Teacher Education, 28(2), 251-262. doi:10.1016/j.tate.2011.10.001

Morgan, P. L., \& Hertzog, C. J. (2001). Designing comprehensive transitions. Principal Leadership: Middle Level Edition, 1(7), 10-16.

Neild, R. C., (2009). Falling off track during the transition to high school: What we know and what can be done. The Future of Children, 19(1), 53-76. doi:10.1353/foc.0.0020

Neild, R. C., Balfanz, R., \& Herzog, L. (2007). An early warning system. Educational Leadership, 65(2), 28.

Neild, R. C., Stoner-Eby, S., \& Furstenberg, F. (2008). Connecting entrance and departure: The transition to ninth grade and high school dropout. Education and Urban Society, 40(5), 543-569. doi:10.1177/0013124508316438

Nelson, H. K., Fairchild, M., Grossenbacher, M., \& Landers, L. (2007). Examining effective middle grades programs: Stating implications for secondary school reform. American Secondary Education, 35(2), 52-68.

Newmann, F. M., Smith, B., Allensworth, E., \& Bryk, A. S. (2001a). Instructional program coherence: What it is and why it should guide school improvement policy. Educational Evaluation and Policy Analysis, 23(4), 297-321. doi:10.3102/01623737023004297 
Newmann, F. M., Smith, B., Allensworth, E., \& Byrk, A. S. (2001b). School instructional program coherence: Benefits and challenges (Descriptive Report (141) ED 451 305). Consortium on Chicago School Research. Retrieved from https://files.eric.ed.gov/fulltext/ED451305.pdf

Ohlsson, J. (2013). Team learning: Collective reflection processes in teacher teams. Journal of Workplace Learning, 25(5), 296-309. doi:10.1108/JWL-Feb-2012-0011

Oakes, A. (2009) Middle-to-high-school transition. Retrieved from: https://files.eric.ed.gov/fulltext/ED506363.pdf

Parrish, T., Poland, L., Arellanes, M., Ernandes, J., \& Viloria, J. (2011). Making the move: Transition strategies at California schools with high graduation rates: (537252012-001) [Data set]. American Psychological Association. doi:10.1037/e537252012-001

Prenger, R., Poortman, C. L., \& Handelzalts, A. (2019). The effects of networked professional learning communities. Journal of Teacher Education, 70(5), 441-452. doi:10.1177/0022487117753574

Ronfeldt, M., Farmer, S., McQueen, K., \& Grissom, J. (2015). Teacher collaboration in instructional teams and student achievement. American Educational Research Journal, $52(3), 475-514$

Roybal, V., Thornton, B., \& Usinger, J. (2014). Effective ninth-grade transition programs can promote student success. Education, 134(4), 475-487.

Şahin, F., \& Cemaloğlu, N. (2015). In-school variables supporting and inhibiting organizational learning in secondary education institutions. Procedia - Social and Behavioral Sciences, 174, 2912-2919. doi:10.1016/j.sbspro.2015.01.1028 
Schlosser, L. (2015). Transition by design: The power of vertical teams. AMLE Magazine 2(8), 18-20. Retrieved from https://fisherpub.sjfc.edu/education_facpub/32

Schulz, K.-P., \& Geithner, S. (2010). Individual and organizational development as interplay: An activity-oriented approach. German Journal of Human Resource Management, 24(2), 130-151. doi:10.1177/239700221002400203

Seidman, I. (2013). Interviewing as qualitative research. (4th ed.). New York, NY: Teachers College Press.

Sekulich, K. M. (2019). Curriculum and assessment alignment mapping. Lutheran Education, 155(2), 33-39.

Slick, S. (2002). Teachers are enthusiastic participants in a learning community. The Clearing House: A Journal of Educational Strategies, Issues and Ideas, 75(4), 198-201. doi:10.1080/00098650209604931

Smith, J. S. (2006a). Examining the long-term impact of achievement loss during the transition to high school. Journal of Secondary Gifted Education, 17(4), 211-221.

Smith, J. S. (2006b). Similarities and differences in students' and parents' perceptions of the transition from middle school to high school. Research in Middle Level Education Online, 29(10), 1-9. doi:10.1080/19404476.2006.11462033.

Smith, T. J. (2007). Managing the transition to ninth grade in a comprehensive urban high school. (National High School Center Report). Retrieved from https://files.eric.ed.gov/fulltext/ED501072.pdf

Smith, J. S., Akos, P., Lim, S., \& Wiley, S. (2008). Student and stakeholder perceptions of the transition to high school. The High School Journal, 91(3), 32-42. doi:10.1353/hsj.2008.0003 
Somers, C. L., Owens, D., \& Piliawsky, M. (2009). A study of high school dropout prevention and at-risk ninth graders' role models and motivations for school completion. Education, 130(2), 348-356.

Sorensen, L. C. (2019). "Big data" in educational administration: An application for predicting school dropout risk. Educational Administration Quarterly, 55(3), 404-446. doi:10.1177/0013161X18799439

Squires, D. (2012). Curriculum alignment research suggests that alignment can improve student achievement. Clearing House, 85(4), 129-135. doi:10.1080/00098655.2012.657723

Statista. (n.d.). Average composite American college testing (ACT) score in the United States in 2019, by state. Retrieved from https://www.statista.com/statistics/305987/us-average-actscores-by-state/

Stosich, E. L., Bocala, C., \& Forman, M. (2018). Building coherence for instructional improvement through professional development: A design-based implementation research study. Educational Management Administration \& Leadership, 46(5), 864-880.

Suh, J., \& Seshaiyer, P. (2015). Examining teachers' understanding of the mathematical learning progression through vertical articulation during lesson study. Journal of Mathematics Teacher Education, 18(3), 207-229. doi:10.1007/s10857-014-9282-7

Uvaas, T., \& McKevitt, B. C. (2013). Improving transitions to high school: A review of current research and practice. Preventing School Failure, 57(2), 70-76. doi:10.1080/1045988X.2012.664580

Vangrieken, K., Dochy, F., Raes, E., \& Kyndt, E. (2015). Teacher collaboration: A systematic review. Educational Research Review, 15, 17-40. doi:10.1016/j.edurev.2015.04.002 
Vera, E., Shriberg, D., Alves, A., Montes de Oca, J., Reker, K., Roche, M., Salgado, M., Stegmaier, J., Viellieu, L., Karahalios, V., Knoll, M., Adams, K., Diaz, Y., \& Rau, E. (2016). Evaluating the impact of a summer dropout prevention program for incoming freshmen attending an under-resourced high school. Preventing School Failure, 60(2), 161-171. doi:10.1080/1045988X.2015.1063039

Wentzel, K. R., Tomback, R., Williams, A., \& McNeish, D. (2019). Perceptions of competence, control, and belongingness over the transition to high school: A mixed-method study. Contemporary Educational Psychology, 56, 55-66. doi:10.1016/j.cedpsych.2018.11.005

Youngs, P., Holdgreve-Resendez, R. T., \& Qian, H. (2011). The role of instructional program coherence in beginning elementary teachers' induction experiences. The Elementary School Journal, 111(3), 455-476. doi:10.1086/657655 


\section{Appendix}

Table 1

Descriptive Statistics of Participating High Schools from the 2018-2019 School Year

\begin{tabular}{lccccc}
\hline & $N$ & Minimum & Maximum & $M$ & $S D$ \\
\hline Current Enrollment & 32 & 97.00 & 1467.00 & 613.69 & 432.27 \\
Free or Reduced Lunch Rate & 32 & 9.50 & 100.00 & 49.28 & 23.37 \\
Graduation Rate & 32 & 78.23 & 100.00 & 92.43 & 5.87 \\
EOC Algebra 1 & 32 & 5.30 & 65.70 & 32.08 & 15.54 \\
EOC English II & 32 & 22.90 & 81.40 & 57.87 & 11.97 \\
EOC Biology & 32 & 15.40 & 57.50 & 37.38 & 11.79 \\
Average Freshman GPA & 26 & 2.30 & 3.50 & 3.01 & 0.33 \\
Freshman Attendance Rate & 28 & 70.00 & 97.20 & 93.29 & 5.84 \\
Dropout Rate & 32 & 0.00 & 5.40 & 1.44 & 1.76 \\
Freshman Course Failure Rate & 27 & 0.10 & 20.00 & 8.67 & 5.81 \\
Freshman OSS Rate & 27 & 0.00 & 15.70 & 4.55 & 4.53 \\
\hline
\end{tabular}


Table 2

Descriptive Statistics of Instructional Program Coherence Predictor Variables

\begin{tabular}{lccccc}
\hline & $N$ & Minimum & Maximum & $M$ & $S D$ \\
\hline Academic Climate & 32 & 2.25 & 3.88 & 3.23 & 0.40 \\
Curriculum & 32 & 1.75 & 4.00 & 3.17 & 0.46 \\
Instruction & 32 & 2.00 & 3.83 & 3.16 & 0.37 \\
Assessment & 32 & 1.75 & 4.00 & 3.09 & 0.53 \\
Vertical Collaboration & 32 & 1.20 & 3.80 & 2.70 & 0.54 \\
\hline Overall IPC & 32 & 1.79 & 3.55 & 3.07 & 0.38 \\
\hline
\end{tabular}

Note. The 4-point Likert scale measures as follows: $1=$ not at all, 2 = some or needs

improvement, $3=$ most or emerging, and $4=$ to great extent or excelling. Adapted from

"Instructional Program Coherence: What It Is and Why It Should Guide School Improvement

Policy,” by F. M. Newmann, B. Smith, E. Allensworth, and A. S. Bryk, 2001, Educational

Evaluation and Policy Analysis, 23, p. 316. Copyright 2001 by the American Educational

Research Association. 
Table 3

Pearson Correlation of IPC and Positive Student Measures from the 2018-2019 School Year

\begin{tabular}{|c|c|c|c|c|c|c|}
\hline & $\begin{array}{c}\text { Graduation } \\
\text { Rate } \\
(N=32) \\
\end{array}$ & $\begin{array}{c}\text { EOC } \\
\text { Algebra } 1 \\
(N=32) \\
\end{array}$ & $\begin{array}{c}\text { EOC } \\
\text { English } 2 \\
(N=32) \\
\end{array}$ & $\begin{array}{c}\text { EOC } \\
\text { Biology } \\
(N=32) \\
\end{array}$ & $\begin{array}{c}\text { Freshmen } \\
\text { Attendance } \\
(N=28)\end{array}$ & $\begin{array}{c}\text { Freshman } \\
\text { GPA } \\
(N=26) \\
\end{array}$ \\
\hline \multicolumn{7}{|c|}{ Instruction } \\
\hline $\begin{array}{c}\text { Pearson } \\
\text { Correlation }\end{array}$ & $.378^{*}$ & .279 & .051 & .071 & .014 & .109 \\
\hline Sig. (2-tailed) & .033 & .122 & .781 & .700 & .942 & .598 \\
\hline \multicolumn{7}{|c|}{ Curriculum } \\
\hline $\begin{array}{c}\text { Pearson } \\
\text { Correlation }\end{array}$ & $.384^{*}$ & .238 & .078 & .125 & .051 & .080 \\
\hline Sig. (2-tailed) & .030 & .190 & .673 & .494 & .795 & .698 \\
\hline \multicolumn{7}{|c|}{ Assessment } \\
\hline $\begin{array}{c}\text { Pearson } \\
\text { Correlation }\end{array}$ & .337 & .230 & -.063 & -.111 & -.122 & -.102 \\
\hline Sig. (2-tailed) & .059 & .205 & .733 & .546 & .538 & 619 \\
\hline \multicolumn{7}{|c|}{ Academic Climate } \\
\hline $\begin{array}{c}\text { Pearson } \\
\text { Correlation }\end{array}$ & .309 & $.435^{*}$ & .171 & .059 & .039 & .014 \\
\hline Sig. (2-tailed) & .085 & .013 & .351 & .747 & .842 & .947 \\
\hline \multicolumn{7}{|c|}{ Vertical Collaboration } \\
\hline $\begin{array}{c}\text { Pearson } \\
\text { Correlation }\end{array}$ & .049 & .160 & -.042 & .095 & .095 & $.542^{* *}$ \\
\hline Sig. (2-tailed) & .788 & .381 & .820 & .604 & .629 & .004 \\
\hline \multicolumn{7}{|c|}{ Overall IPC } \\
\hline $\begin{array}{c}\text { Pearson } \\
\text { Correlation }\end{array}$ & .341 & .314 & .035 & .053 & .015 & .175 \\
\hline Sig. (2-tailed) & .056 & .080 & .850 & .775 & .938 & .392 \\
\hline
\end{tabular}

*** Correlation is significant at the 0.01 level (2-tailed).

* Correlation is significant at the 0.05 level (2-tailed). 
Table 4

Linear Regression of Overall IPC on Positive Student Outcomes when Controlling for Socioeconomic Status

\begin{tabular}{|c|c|c|c|c|c|}
\hline Dependent Variable & $R$ & $R^{2}$ & Adjusted $R^{2}$ & $R^{2}$ Change & Significance \\
\hline \multirow[t]{2}{*}{ Graduation Rate } & $.428^{\mathrm{a}}$ & .183 & .156 & .183 & .015 \\
\hline & $.526^{\mathrm{b}}$ & .277 & .227 & .094 & .062 \\
\hline \multirow[t]{2}{*}{ EOC Algebra 1} & $.302^{\mathrm{a}}$ & .091 & .061 & .091 & .093 \\
\hline & $.419^{b}$ & .175 & .119 & .084 & .096 \\
\hline \multirow[t]{2}{*}{ EOC English 2} & $.655^{\mathrm{a}}$ & .429 & .410 & .429 & .000 \\
\hline & $.655^{\mathrm{b}}$ & .430 & .390 & .000 & .889 \\
\hline \multirow[t]{2}{*}{ EOC Biology } & $.526^{\mathrm{a}}$ & .276 & .252 & .276 & .002 \\
\hline & $.526^{\mathrm{b}}$ & .276 & .226 & .000 & .956 \\
\hline \multirow[t]{2}{*}{ Average Freshman GPA } & $.445^{\mathrm{a}}$ & .198 & .165 & .198 & .023 \\
\hline & $.463^{\mathrm{b}}$ & .214 & .146 & .016 & .504 \\
\hline \multirow[t]{2}{*}{ Freshman Attendance } & $.522^{\mathrm{a}}$ & .273 & .245 & .273 & .004 \\
\hline & $.524^{\mathrm{b}}$ & .274 & .216 & .002 & .818 \\
\hline
\end{tabular}

a. Predictors: (Constant), Percentage of students on free or reduced lunch

b. Predictors: (Constant), Percentage of students on free or reduced lunch, Overall IPC 
Table 5

Pearson Correlation of IPC and Negative Student Measures from the 2018-2019 School Year

\begin{tabular}{|c|c|c|c|}
\hline & $\begin{array}{l}\text { Dropout Rate } \\
\qquad(N=32)\end{array}$ & $\begin{array}{c}\text { Freshmen Course } \\
\text { Failure Rate } \\
(N=27) \\
\end{array}$ & $\begin{array}{l}\text { Freshmen OSS Rate } \\
\qquad(N=27)\end{array}$ \\
\hline \multicolumn{4}{|c|}{ Instruction } \\
\hline Pearson Correlation & -.320 & .066 & .134 \\
\hline Sig. (2-tailed) & .074 & .743 & .505 \\
\hline \multicolumn{4}{|c|}{ Curriculum } \\
\hline Pearson Correlation & -.312 & .017 & .241 \\
\hline Sig. (2-tailed) & .082 & .932 & .226 \\
\hline \multicolumn{4}{|c|}{ Assessment } \\
\hline Pearson Correlation & -.258 & .224 & .110 \\
\hline Sig. (2-tailed) & .153 & .261 & .585 \\
\hline \multicolumn{4}{|c|}{ Academic Climate } \\
\hline Pearson Correlation & $-.351^{*}$ & .106 & .245 \\
\hline Sig. (2-tailed) & .049 & .600 & .219 \\
\hline \multicolumn{4}{|c|}{ Vertical Collaboration } \\
\hline Pearson Correlation & -.003 & -.025 & .062 \\
\hline Sig. (2-tailed) & .985 & .900 & .758 \\
\hline \multicolumn{4}{|c|}{ Overall IPC } \\
\hline Pearson Correlation & -.286 & .110 & .187 \\
\hline Sig. (2-tailed) & .113 & .584 & .349 \\
\hline
\end{tabular}

*** Correlation is significant at the 0.01 level (2-tailed).

* Correlation is significant at the 0.05 level (2-tailed). 
Table 6

Linear Regression of Overall IPC on Negative Student Outcomes when Controlling for Socioeconomic Status

\begin{tabular}{lccccc}
\hline \multicolumn{1}{c}{ Dependent Variable } & $R$ & $R^{2}$ & Adjusted $R^{2}$ & $R^{2}$ Change & Significance \\
\hline Dropout Rate & $.423^{\mathrm{a}}$ & .179 & .152 & .179 & .016 \\
& $.492^{\mathrm{b}}$ & .243 & .190 & .063 & .131 \\
\hline $\begin{array}{l}\text { Freshman Course Failure } \\
\text { Rate }\end{array}$ & $.282^{\mathrm{a}}$ & .080 & .043 & .080 & .154 \\
& $.314^{\mathrm{b}}$ & .099 & .024 & .019 & .481 \\
\hline Freshman OSS Rate & $.339^{\mathrm{a}}$ & .115 & .079 & .115 & .084 \\
& $.408^{\mathrm{b}}$ & .167 & .097 & .052 & .234 \\
\hline
\end{tabular}

a. Predictors: (Constant), Percentage of students on free or reduced lunch

b. Predictors: (Constant), Percentage of students on free or reduced lunch, Overall IPC 
SECTION SIX: SCHOLARLY PRACTITIONER REFLECTION 
Do you remember your high school yearbook quote? If you do, it is usually because it is unique or silly. Maybe you remember it because it reminds you of someone you shared your high school experience with or a particular event you attended. I remember mine, but not for any of those reasons. I remember mine because of how relevant it resonates in my personal and professional life today. My quote was stolen from the great Vince Lombardi who once stated, "Those who invest the most are the last to surrender." Throughout the dissertation process, I kept referring to this quote. Of course, the investment of time and resources apply on the surface, but to me this was so much more. Investing in this dissertation will have a lasting effect on me as a leader and a scholar, an effect that I plan to utilize for as long as I can. The doctorate program in its entirety has been a journey of self-discovery that made me constantly reflect and change who I am as a person, an educator, and a leader. The dissertation continued that reflective journey.

\section{Dissertation Influence on Being an Educational Leader}

The dissertation was the culmination of the hard work and learning that took place throughout the doctorate program. A level of independence was introduced in the dissertation that required me to grow into the educational leader I hoped to become. Upon reflection, I determined that the dissertation process reaffirmed the vital role of relationships and empathy in leadership, illustrated the importance of self-care, and forced me to evaluate and address benefits and pitfalls of my own ambition. The following is an outline of how the dissertation process specifically influenced me as an educational leader.

The aspect that stands out the most in my experience with the dissertation was how much my impression of leadership has changed over the course of the doctorate journey. Ironically, it was in searching for new knowledge in my study that made me reaffirm that while valuable, I 
overvalued expertise. It also illuminated my best transformation through the doctorate of how I value relationships in leadership. When I took the Gallup Strengths Test at the beginning of the program, I was somewhat disturbed at what it revealed to me. I was shocked to find out that some of my strengths were notoriety driven and seemed self-centered. What was worse is that after self-examination, they were extremely accurate and void of any relationship strengths which I always used to pride myself in having. Fortunately, the doctorate was meant to be transformative, and I have improved the most in that area.

The one area of relationship building that I can pinpoint as being reinforced the most was the use of empathy. This was illustrated due to the unique situation imposed upon us during this time due to Covid-19. It is human nature for us to tend to live in our own little bubbles. Trying to complete a dissertation during the pandemic magnified this for me. I had found a topic, that due to my position as a freshman teacher, I was very passionate about and felt a real need to pursue. It was exciting to be the first to delve into a topic that had never been specifically researched before and the thought of how it could eventually help students like the ones I teach was so rewarding. Thus, I was disappointed with the extremely low participation rate I received. The lack of response from high school principals around the state, ones I aspired to possibly join one day, felt personal at times. It seemed to diminish the importance of the topic I believed could really contribute to advance high school education. At times it made me question everything I was trying to do, and if I was really meant to be a researcher, or for that matter a leader in education.

Under normal circumstances, a low response rate might imply that there is not a need for to focus on building IPC between teachers in high schools and their feeder schools. That still may be. I would be satisfied if that were the conclusion drawn through valid research. However, 
I had to remind myself this was not the case with my study. Covid-19 forced these principals into unprecedented situations that required their utmost attention. They had to do what great leaders do, prioritize what they could focus on in a time of need. Most were balancing the health and learning of their students, the well-being of their families and communities, and navigating through unknown ever-changing terrain of which they had little control. In doing my research, I stepped out of my little bubble and empathized with them. I found that the work they were doing in this time was nothing short of amazing. Being a teacher facing similar challenges, I understood why so many did not participate. They simply could not. For some to take the time to complete a less than convenient survey was admirable. Others even took the time out to show me support, whether they participated or not. I am grateful to have witnessed such selfless leadership from some of these principals and hope to emulate that moving forward.

Being a researcher during this time was frustrating. Honestly, I am a little ashamed to admit it changed my focus from making a difference with my research to just getting it finished. I do not feel shame in this anymore, as I know the situation was unique and that I can conduct further research in the future that will make a difference for incoming freshmen. More importantly, I learned a lot about handling the obstacles as an educational leader, and at times, unforeseen problems will require tough sacrifices. Completing this dissertation during such turbulent times allowed me to learn the importance of perseverance during adversity. It is not cliché that goals worth achieving do not come easy. This adversity has made me much stronger as a person, and I feel will benefit me greatly as an educational leader.

Witnessing the great educators persevere around me during this process illustrated another important aspect of leadership I have neglected in the past, physical and mental self-care. A common theme among these leaders was they maintained a healthy balance in their lives. 
Most maintained the physical fitness that allowed for them to perform effectively under stressful circumstances. More impressive was how they managed the psychological strain that adversity can put on a person. Even before Covid-19, I faced personal hardships that derailed my progress on numerous occasions. The dissertation process can be extremely stressful and has conquered many people in the past, but I always scoffed at the idea that it would happen to me. During the beginning of the process that laughable idea became closer to a reality. Then the pandemic hit.

What I found in dealing with my own mental and physical health was imperative for me to learn moving forward as an educational leader. Great leaders often draw inspiration and get help from others to survive and thrive. While almost succumbing to my own adversity, I witnessed those around me conquering situations far more challenging or dire than mine. Not only were those leaders able to maintain their own well-being, they were empowering others to do the same. It was truly motivating to see. Personally, some close to me put the events of their own lives, some tragic, on hold to help get me back on track. I had reached some of the lowest points of my life at the worst possible timing while trying to complete a doctorate. Through them I was able to change my perspective of getting help as a weakness to being one of the most courageous things someone can do. Without their example or assistance, I still maintain that it probably would not have come to fruition.

Some may feel worrying about you own well-being may come off as being self-centered. The dissertation process has made me argue that it is the exact opposite. Leaders in education tend to have an innate desire to be of service to others and pride themselves in doing so. I believe the most ethical educational leaders unconsciously use this as their driving motivation for every action or decision. However, the desire to help only becomes an ability when one has the internal means to follow through. One cannot give if their cup is empty. Great educational 
leaders prioritize self-care and self-preservation to help those around them. I had to come to tough realizations in order to do the same in hopes to complete research that will someday benefit others.

The idea of helping others leads me to the final major lesson that this dissertation has taught me which is to continuously examine my own ambition. Two of my strengths were restorative and responsibility (Gallup, 2016). This combination leads to ambition to look for and fix problems, but also to take on too much. When deciding on a study that fit my topic, I was extremely ambitious with what I was capable of accomplishing. My goal was to expose the problem I perceived as academic incoherence between mid-level and high school teachers and then fix it with one study. It was a noble idea, yet an ignorant one as well.

Another strength of mine that came into play during this time was that of self-assurance. Clifton, Anderson, and Schreiner (2006) stated that one aspect of the self-assurance is:

When you look at the world, you know that your perspective is unique and distinct. And because no one sees exactly what you see, you know that no one can make your decisions for you. No one can tell you what to think. They can guide. They can suggest. But you alone have the authority to form conclusions, make decisions, and act. (p. 3)

With how I have changed over the course of the doctorate, I now consider this a negative as I feel I am much stronger when I accept the input of others. Yet at the beginning of the dissertation process this characteristic of my self-assurance once again imposed itself and made the process both frustrating and disorienting. In my mind I felt I knew what the best study was and rarely took the suggestions of others positively.

The challenge brought about by the dissertation also made me question my capabilities, which was difficult to accept. I could not conquer this topic alone. I did not yet have the tools or 
knowledge to conduct a study to bring about change. Although the worst realization was that what I was experiencing as a practitioner created such a strong bias that this was an issue in education that needed to be addressed, but maybe this was not the case. I could not accept that the research could potentially disagree with my way of thinking. My ambition and selfassurance blinded me to the harsh realities I was facing. To persevere, I needed to put to practice different aspects of leadership I had learned from the doctorate program: reframe my perspective, agree to receive help from others, admit my biases and account for them, and accept that the best outcome may not be the anticipated or preferred one.

Ambition is not always a negative trait. Theoharis (2010) introduced an idea of educational leaders being passionate visionaries. While his article was related to social justice, characteristics of passionate visionaries relate to much more and are ones I want to emulate. They are so committed, enthusiastic, and caring that their passion is embedded in the fiber of their character (Theoharis, 2010). Passionate visionaries are relentless in their pursuit for progress in education and change people's self-centered beliefs to help others (Theoharis, 2010). I feel the optimism and self-confidence leaders possess that allows for them to create lasting change in education would not be possible without their ambition to undertake such a task.

When used responsibly, ambition unlocks many qualities that allows educational leaders to be successful such as taking calculated risks and inspiring hope in others. I feel I share that same ambition. During the dissertation process I was once told, "first become a doctor, then change the world." While the title of doctor is beneficial in many ways, the benefit I covet the most is accessibility to help create change. Moving education forward is a passion of mine that I am determined to pursue. My ambition to complete the dissertation allowed me to do so and 
produced in me the same self-confidence passionate visionaries possess. On a more concrete level, it provided me with another avenue to create positive change, through scholarship.

\section{Dissertation Influence on Being a Scholar}

As influential as the dissertation process was on me as an educational leader, it was arguably more influential on me as a scholar. One thing I have learned is that while being a leader and scholar can be mutually exclusive, they can also work harmoniously to maximize a person's impact in education. When reflecting on the effect the dissertation had on me as a scholar, there are two areas it has influenced me the most. They are as follows: (a) how academia has unlocked an entire world of possibilities to move education forward, and (b) the lessons I learned that I will apply to all the future research I plan to pursue.

Being a passionate visionary is an integral part of who I want to be as an educational leader. Certain aspects of the current education system in this country is stagnant and outdated. Before the doctorate, I always had the interest to but to pursue solutions to the problems facing education but did not feel I possessed the capability. I also felt that a status or position was required to achieve those difficult goals. Academic research provides the avenue to take on those tasks regardless of title. What better way to be a passionate visionary in education than to contribute new knowledge that will move the field forward. The doctorate classwork exposed to me the elements of the academic world. The dissertation process unlocked my ability and passion to be a part of that world. If solutions exist that can help students, staffs, and communities, who wouldn't want to pursue them? My goal is to not only one day be published, but to know that like my impact as a teacher, my impact as a researcher has benefited others.

The dissertation process also taught me about the access academic writing can bring to an educator. I often thought about how many teachers, professors, counselors, and administrators 
out there are doing innovative work that is overlooked because it does not have the audience or credence it deserves. The rigorous process of completing peer reviewed research automatically results in the growth of credibility and the likelihood its content is applied in a broader environment. Unbeknownst to me at the time, when I was reading the academic work of others, I was becoming an expert on the transition to high school. I came across so many concepts that I believed in because of the source and applied it without hesitation in my career to help the current and future students of our high school. It also led me to look to academic research to improve my practice in other facets. I now read every educational article with the same question in the back of my mind, "Is there any way I can take this idea further?" It is an exciting thought to not only continue research in the academic arena, but also to have the confidence in my abilities to do so. This can all be attributed to the dissertation process.

Early in my career I never would have imagined pursuing a doctorate or contributing to research. Now, I can hardly imagine not continuing research moving forward. The journey I have made as a scholar has been transformational. Before the doctorate, I cannot remember ever doing any academic reading or writing. I did not even know what a peer reviewed journal article was. My first paper I wrote in the program was harshly panned by one of the most brilliant professors I have ever encountered. He called me a storyteller and not an academic writer. As harsh as that seemed at the time, looking back I am unbelievably thankful he gave me that advice in the manner he did. The professors in the program are so admirable and I want to emulate them so badly that I used that criticism as motivation and learned from it. I was so self-conscious when it came to my abilities in this new community. But if I wanted to be one of those doctors, I had to adapt. Fortunately, my parents instilled in me a work ethic that I used to compensate. I would read articles multiple times to fully understand the content. All of this contributed to the 
dissertation capstone that was looming and hopefully acceptance into a world I so badly wanted to join.

While I learned so much about academic reading and writing, the dissertation brought about lessons that I will carry with me in all future research. Although when unharnessed it sometimes worked against me, one thing I found beneficial overall is that I am my own harshest critic. This allowed me to unsympathetically examine my own shortcomings as a researcher and academic writer and accept constructive criticism. My improvement in writing has been immense but moving forward I must be attentive to precision. I tend to compensate for perceived knowledge inadequacy by being unnecessarily voluminous in my writing, by being both too wordy and including needless information. When doing academic reading, there was a glaring disparity in the precision in their work compared to mine.

Another lesson that I learned was to have a plan and a system and stick to it. Throughout the process I was always looking to streamline the way I was working, sometimes through tricks and tips I would find. This often led to me expending inordinate amounts of time and energy changing to methods that were counterproductive and disorganized. It made the duration of the dissertation much longer than necessary. Of course, one should always look to improve the way they research, but their motivation should not be to avoid the hard work it requires. With that as my motivation, I also learned another hard lesson. While one should not be afraid to ask for help, they should not over-rely on others for efficiency purposes. When finding my population to disseminate my survey, I relied on a filtration system in a DESE directory recommended to me by one of their employees. It was not until I sent out paper surveys that I realized this directory's filtration system was inaccurate and was also not updated. This led to me doing hours of extra work to rectify my error of blindly believing the DESE employee. 
The themes from the lessons I learned led to one conclusion that will be at the forefront of my mindset with academic research. The work is hard and unforeseen obstacles will occur that will test my will as a researcher. To alleviate this as much as possible, I must be more intentional with preparation and more meticulous with my work. Pushing too fast to save time is a gamble that rarely pays off as you waste more time re-doing work that failed to meet academic standards. I plan on doing a better job of foreshadowing possible outcomes of my decisions before I make them. This should allow for me to focus more attention on the content and on areas where I can improve rather than wasting time on corrections. Towards the end of my dissertation, all my previous mistakes made me intentionally prioritize a different mindset that had me questioning the validity of every aspect of my study. To reach the aspirations I want to in academic research, I need to raise the standard of work I produce. To do so requires I continuously learn from my previous work on this dissertation.

\section{Conclusion}

Unlike many others, this dissertation was not only a culminating project for me to obtain a doctorate. Upon entrance of the program, that was my impression. That dissipated over time but vanished completely when actually completing it. Completing the dissertation has given me the knowledge, skills, and confidence to pursue the thing in education I am most passionate about, moving the field forward in hopes of helping others live better lives. Because of that this dissertation was not a final project, but a springboard to the future. Most importantly, this doctorate was an investment in myself and others. 


\section{References}

Akiba, M. (2012). Professional learning activities in context: A statewide survey of middle school mathematics teachers. Education Policy Analysis Archives, 20(14). doi:10.14507/epaa.v20n14.2012

Akos, Patrick., \& Galassi, J. P. (2004). Middle and high school transitions as viewed by students, parents, and teachers. Professional School Counseling, 7(4), 212-221.

Allensworth, E. M., \& Easton, J. Q. (2007). What matters for staying on-track and graduating in Chicago public high schools: A close look at course grades, failures, and attendance in the freshman year. Research Report. Consortium on Chicago School Research. Retrieved from https://files.eric.ed.gov/fulltext/ED498350.pdf

Alspaugh, J. W. (1998). Achievement loss associated with the transition to middle school and high school. Journal of Educational Research, 92(1), 20.

Anderman, E. M., \& Midgley, C. (2004). Changes in self-reported academic cheating across the transition from middle school to high school. Contemporary Educational Psychology, 29(4), 499-517.

Anderson, R. C., Graham, M., Kennedy, P., Nelson, N., Stoolmiller, M., Baker, S. K., \& Fien, H. (2019). Student agency at the crux: Mitigating disengagement in middle and high school. Contemporary Educational Psychology, 56, 205-217.

doi:10.1016/j.cedpsych.2018.12.005 
Anderson, R., Styron, R.A., Roberson, T., \& Rachal, J. (2005) The journals that administrators read. Educational leadership. Retrieved from http://www.ascd.org/publications/educationalleadership/summer05/vol62/num09/Special-Topic@-The-Journals-That-AdministratorsRead.aspx

Arensman, B., van Waegeningh, C., \& van Wessel, M. (2018). Twinning "practices of change” with "theory of change": Room for emergence in advocacy evaluation. American Journal of Evaluation, 39(2), 221-236.

Balfanz, R. (2011). Back on track to graduate. Educational Leadership, 68(7), 54-58.

Balfanz, R., Herzog, L., \& Mac Iver, D. J. (2007). Preventing student disengagement and keeping students on the graduation path in urban middle-grades schools: Early identification and effective interventions. Educational Psychologist, 42(4), 223-235. doi:10.1080/00461520701621079

Baydu, M. M., Kaplan, O., \& Bayar, A. (2013). Facing the influence of poverty on graduation rates in public high schools. Procedia - Social and Behavioral Sciences, 84, 233-237. doi:10.1016/j.sbspro.2013.06.541

Beckham, J., Klaymeier Willis, B., \& Weeks, K. M. (2020). Responsibilities duties decisionmaking and legal basis for local school board powers. Retrieved from https://education.stateuniversity.com/pages/2391/School-Boards.html

Belton School District \#124. (2020). The academies of Belton. Retrieved from https://www.beltonschools.org/domain/384

Benner, A. D. (2011). The transition to high school: current knowledge, future directions. Educational Psychology Review, 23(3), 299. doi:10.1007/s10648-011-9152-0 
Benner, A. D., Boyle, A., \& Bakhtiari, F. (2017). Understanding students' transtion to high school: Demographic variation and the role of supportive relationships. Journal of Youth \& Adolescence, 46(10), 2129-2142. https://doi-org. /10.1007/s10964-017-0716-2

Benner, A. D. \& Graham, S. (2009), The transition to high school as a developmental process among multiethnic urban youth. Child Development, 80, 356-376. doi:10.1111/j.14678624.2009.01265.x

Benner, A. D., \& Wang, Y. (2014). Demographic marginalization, social integration, and adolescents' educational success. Journal of Youth \& Adolescence, 43(10), 1611-1627. doi:10.1007/s10964-014-0151-6

Bergman, D., Calzada, L., LaPointe, N., Lee, A., \& Sullivan, L. (1998). Vertical alignment and collaboration (Doctoral dissertation, Texas A\&M University, Corpus Christi/Kingsville). Retrieved from https://files.eric.ed.gov/fulltext/ED421472.pdf

Berry, B., Daughtrey, A., \& Wieder, A. (2009, December). Collaboration: Closing the effective teaching gap. Center for Teaching Quality.

Bertrand, L., Roberts, R. A., \& Buchanan, R. (2006). Striving for success: Teacher perspectives of a vertical team initiative. National forum of teacher education journal-electronic $16(3), 1-10$.

Biermann, B.R. (2010). A descriptive study of public high school freshmen transition programs in the Kansas City metropolitan area (Doctoral dissertation, University of Kansas, Lawrence, KS). Retrieved from https://kuscholarworks.ku.edu/bitstream/handle/1808/6748/Biermann_ku_0099D_10953_ DATA_1.pdf?isAllowed=y\&sequence $=1$ 
Birkhead, S., Suh, J., Gerasimova, D., \& Seshaiyer, P. (2017). Improving knowledge of algebraic learning progressions through professional learning in collaborative vertical teams. Professional Development, 8, 415-422.

Bjerk, D. (2012). Re-examining the impact of dropping out on criminal and labor outcomes in early adulthood. Economics of Education Review, 31(1), 110-122. doi:10.1016/j.econedurev.2011.09.003

Bolman, L. G., \& Deal T. E. (2010). Reframing the path to school leadership: A guide for teachers and principals (2nd ed., pp. 83-94). Thousand Oaks, CA: Corwin.

Bolman, L. G., \& Deal T. E. (2013). Reframing organizations: Artistry, choice, and leadership. San Francisco, CA: Jossey-Bass

Bornsheuer, J. N., Polonyi, M. A., Andrews, M., Fore, B., \& Onwuegbuzie, A. J. (2018). The relationship between ninth-grade retention and on-time graduation in a Southeast Texas high school. The Journal of At-Risk Issues, 16(2), 9.

Brake, A. (2020). Right from the start: Critical classroom practices for building teacher-student trust in the first 10 weeks of ninth grade. The Urban Review, 52(2), 277-298. doi:10.1007/s11256-019-00528-z

Brown, L. C. \& Seeley, C. L. (2010). Transitions from middle school to high school: crossing the bridge. Mathematics Teaching in the Middle School, 15(6), 354-358.

Butts, M. J., \& Cruzeiro, P. A. (2005). Student perceptions of factors leading to an effective transition from eight to nine grade. American Secondary Education, 34(1), 70.

Campbell, C. (2015). High school dropouts after they exit school: Challenges and directions for sociological research. Sociology Compass, 9(7), 619-629. doi:10.1111/soc4.12279 
Campbell, C., \& Levin, B. (2009). Using data to support educational improvement. Educational Assessment, Evaluation and Accountability, 21(1), 47-65. doi:10.1007/s11092-008-9063$\mathrm{x}$

Casillas, A., Robbins, S., Allen, J., Kuo, Y.-L., Hanson, M. A., \& Schmeiser, C. (2012). Predicting early academic failure in high school from prior academic achievement, psychosocial characteristics, and behavior. Journal of Educational Psychology, 104(2), 407-420. doi:10.1037/a0027180

Cauley, K. M., \& Jovanovich, D. (2006). Developing an effective transition program for students entering middle school or high school. Clearing House, 80(1), 15-25. doi:10.3200/TCHS.80.1.15-25

Cavendish, W. (2013). Student perceptions of school efforts to facilitate student involvement, school commitment, self-determination, and high school graduation. Social Psychology of Education, 16(2), 257-275. doi:10.1007/s11218-013-9212-z

Chapman, C., Laird, J., Ifill, N., \& Kewal Ramani, A. (2011). Trends in High School Dropout and Completion Rates in the United States: 1972-2009. Compendium Report. NCES 2012-006. National Center for Education Statistics. Retrieved from http://nces.ed.gov/pubsearch.

Chapman, M. V., \& Sawyer, J. S. (2001). Bridging the gap for students at risk of school failure: A social work-initiated middle to high school transition program. Children \& Schools, 23(4), 235. doi:10.1093/cs/23.4.235

Chatalalsingh, C., \& Reeves, S. (2014). Leading team learning: What makes interprofessional teams learn to work well? Journal of Interprofessional Care, 28(6), 513-518. 
Clayton High School. (2020). Ninth-grade and new student transition. Retrieved from https://www.claytonschools.net/Page/16774

Clifton D. O., Anderson E. C., \& Schreiner, L. A. (2006). StrengthsQuest: discover and develop your strengths in academics, career and beyond [Adobe Digital Edition Version]. Retrieved from https://www.strengthsquest.com/208718/strengthsquest.aspx

Cohen, D. K., \& Neufeld, B. (1981). The failure of high schools and the progress of education. Daedalus, 110(3), 69-89.

Cohen, J. S., \& Smerdon, B. A. (2009). Tightening the dropout tourniquet: Easing the transition from middle to high school. Preventing School Failure, 53(3), 177. doi:10.3200/PSFL.53.3.177-184

Cooney, S., \& Bottoms, G. (2003). Middle grades to high school: Mending a weak link. (Research Brief No. 143). Department of Education, Southern Regional Education Board. Retrieved from https://www.sreb.org/programs/hstw/publications/briefs/Middle_Grades_To_HS.pdf

Cooper, R., \& Liou, D. D. (2007). The structure and culture of information pathways: Rethinking opportunity to learn in urban high schools during the ninth-grade transition. High School Journal, 91(1), 43. doi:10.1353/hsj.2007.0020

Corcoran, T., \& Silander, M. (2009). Instruction in high schools: The evidence and the challenge. The Future of Children, 19(1), 157-183. doi:10.1353/foc.0.0026

Crawford, S. T. (2011). Increasing instructional capacity through vertical teaming. Retrieved from https://rb.gy/vqi6wl

Creswell, J. W. (2014). Research design: Qualitative, quantitative, and mixed methods approaches (4th ed.). Thousand Oaks, CA: Sage. 
Crossan, M. M., Lane, H. W., \& White, R. E. (1999). An Organizational learning framework: From intuition to institution. Academy of Management Review, 24(3), 522-537. doi:10.5465/AMR.1999.2202135

Datnow, A., \& Park, V. (2014). Data-driven leadership. San Francisco, CA: Jossey-Bass.

Datnow, A., Park, V., \& Kennedy-Lewis, B. (2013). Affordances and constraints in the context of teacher collaboration for the purpose of data use. Journal of Educational Administration, 51(3), 341-362. doi:10.1108/09578231311311500

Davis, H. A., Chang, M.-L., Andrzejewski, C. E., \& Poirier, R. R. (2014). Examining relational engagement across the transition to high schools in three US high schools reformed to improve relationship quality. Learning Environments Research, 17(2), 263-286. doi:10.1007/s10984-013-9148-4

Davis-Kean, P. E., Huesmann, L. R., Jager, J., Collins, W. A., Bates, J. E., \& Lansford, J. E. (2008). Changes in the relation of self-efficacy beliefs and behaviors across development. Child Development, 79(5), 1257-1269. doi:10.1111/j.1467-8624.2008.01187.x de Wit, L., van Straten, A., Lamers, F., Cuijpers, P., \& Penninx, B. (2011). Are sedentary television watching and computer use behaviors associated with anxiety and depressive disorders? Psychiatry Research, 186(2-3), 239-243. doi:10.1016/j.psychres.2010.07.00

Dedmond, R., Brown, R. D., \& LaFauci, J. M. (2006). Freshman transition programs: Long-term and comprehensive. Principal's Research Review, 1(4), 1-8.

DeLamar, S., \& Brown, C. G. (2016). Supporting transition of at-risk students through a freshman orientation model. Journal of At-Risk Issues, 19(2), 32-39. 
Donohoo, J., \& Katz, S. (2019). What drives collective efficacy? Effective teams that believe they can make a difference create the conditions to get better in four key ways. Educational Leadership, 76(9), 24.

Dorman, B. (2012). The supported teen: Transitioning to high school. Leadership, 41(5), 22-23.

Dupéré, V., Dion, E., Leventhal, T., Archambault, I., Crosnoe, R., \& Janosz, M. (2018). High school dropout in proximal context: The triggering role of stressful life events. Child Development, 89(2), e107-e122. doi:10.1111/cdev.12792

Eccles, J. S., \& Roeser, R. W. (2011). Schools as developmental contexts during adolescence. Journal of Research on Adolescence, 21(1), 225-241. doi:10.1111/j.15327795.2010.00725.x

Egodawatte, G., McDougall, D., \& Stoilescu, D. (2011). The effects of teacher collaboration in Grade 9 Applied Mathematics. Educational Research for Policy and Practice, 10(3), 189-209. doi:10.1007/s10671-011-9104-y

Ellerbrock, C. R., \& Kiefer, S. M. (2014). Supporting young adolescents' middle-to-high-school transition by creating a ninth-grade community of care: Implications for middle grades educators. Middle School Journal, 45(3), 3-10. doi:10.1080/00940771.2014.11461886

Emmett, J., \& McGee, D. (2012). A farewell to freshmen. Clearing House, 85(2), 74-79. doi:10.1080/00098655.2011.619592

Felmlee, D., McMillan, C., Inara Rodis, P., \& Osgood, D. W. (2018). Falling behind: Lingering costs of the high school transition for youth friendships and grades. Sociology of Education, 91(2), 159-182. doi:10.1177/0038040718762136

Field, A. (2018). Discovering statistics using IBM SPSS statistics (5th ed.). Thousand Oaks, CA: Sage. 
Fink, A. (2017). How to conduct surveys: A step-by-step guide (6th ed). Thousand Oaks, CA: Sage.

Finn Jr, C. E. (2006). Obstacles on the route from high school to college. Chronicle of Higher Education, 52(27), 40-42.

Fort Zumwalt East High School. (2020). Freshman transition day - class of 2023. Retrieved from https://ehs.fz.k12.mo.us/news/archived_news/freshman_transition_day_-_class_of_2023

Fulk, B. M. (2003). Concerns about ninth-grade students' poor academic performance: One school's action plan. American Secondary Education, 31(2), 8-26

Gajda, R., \& Koliba, C. J. (2008). Evaluating and improving the quality of teacher collaboration: A field-tested framework for secondary school leaders. NASSP Bulletin, 92(2), 133-153. doi:10.1177/0192636508320990

Galla, B. M., Tsukayama, E., Park, D., Yu, A., \& Duckworth, A. L. (2020). The mindful adolescent: Developmental changes in nonreactivity to inner experiences and its association with emotional well-being. Developmental Psychology, 56(2), 350-363. doi:10.1037/dev0000877

Gallup, Inc. (2016). Insights Report. Washington, D.C.: The Gallup Organization.

Ganeson, K., \& Ehrich, L. C. (2009). Transition into high school: A phenomenological study. Educational Philosophy \& Theory, 41(1), 60-78. doi:10.1111/j.1469-5812.2008.00476.x

Gentle-Genitty, C. (2009). Best practice program for low-income African American students transitioning from middle to high school. Children \& Schools, 31(2), 109-117. doi:10.1093/cs/31.2.109

Gilmer, P. J. (2010). Vertical teaming: K-12 teachers engaged in scientific research in rural settings. The Rural Educator, 31(3), 1-6. 
Goddard, R., Goddard, Y., Sook Kim, E., \& Miller, R. (2015). A theoretical and empirical analysis of the roles of instructional leadership, teacher collaboration, and collective efficacy beliefs in support of student learning. American Journal of Education, 121(4), 501-530. doi:10.1086/681925

Goddard, Y., \& Kim, M. (2018). Examining connections between teacher perceptions of collaboration, differentiated instruction, and teacher efficacy. Teachers College Record, 120(01301), 1-24.

Goldin, C. (1998). America's graduation from high school: The evolution and spread of secondary schooling in the twentieth century. The Journal of Economic History, 58(2), 345-374. doi:10.1017/S0022050700020544

Goldin, C., \& Katz, L. F. (1997). Why the United States led in education: Lessons from secondary school expansion, 1910 to 1940 (No. w6144). Cambridge, MA: National Bureau of Economic Research.

Gruenert, S. (2005). Correlations of collaborative school cultures with student achievement. NASSP Bulletin, 89(645), 43-55. doi:10.1177/019263650508964504

Hadderman, M. (1988). State vs. local control of schools (No. ED291164; ERIC Digest Series Number 24). ERIC Clearinghouse on Educational Management.

Hazel, C. E., Pfaff, K., Albanes, J., \& Gallagher, J. (2014). Multi-level consultation with an urban school district to promote 9th grade supports for on-time graduation. Psychology in the Schools, 51(4), 395-420. doi:10.1002/pits.21752

Head, B. W., \& Alford, J. (2015). Wicked problems: Implications for public policy and management. Administration \& Society, 47(6), 711-739. 
Heifetz, R. A., \& Laurie, D. L (2011). The work of leadership. In Harvard Business Review (Ed.), HBR's 10 Must Reads: On Leadership (pp. 57-78). Brighton, MA: Harvard Business Press.

Henderson, C. (2000). Vertical teams yield vertical achievement in Southeastern Texas school district. Education in Practice, 9, 1-4.

Herlihy, C., \& Kennelly, L. (2007, May). State and district support for successful transitions into high school. Washington, DC: National High School Center

Hickman, G. P., Bartholomew, M., Mathwig, J., \& Heinrich, R. S. (2008). Differential developmental pathways of high school dropouts and graduates. Journal of Educational Research, 102(1), 3-14. https://doi-org/10.3200/JOER.102.1.3-14

Horwitz, A., \& Snipes, J., (2008). Research brief: Supporting successful transitions to high school. Washington, DC: The Council of Greater City Schools

Huerta, J., J., Watt, K. M., \& Butcher, J. T. (2013). Examining Advancement Via Individual Determination (AVID) and its impact on middle school rigor and student preparedness. American Secondary Education, 41(2), 24-37.

Ismail, S. N., Kanesan, A. G., \& Muhammad, F. (2018). Teacher collaboration as a mediator for strategic leadership and teaching quality. International Journal of Instruction, 11(4), 485-498. doi:10.12973/iji.2018.11430a

Independence School District. (2020). Freshman academy. Retrieved from http://sites.isdschools.org/program_of_studies_19-20/freshmen-academy

Jackson, C. K., \& Bruegmann, E. (2009). Teaching students and teaching each other: The importance of peer learning for teachers. American Economic Journal: Applied Economics, 1(4), 85-108. doi:10.1257/app.1.4.85 
Jacobson, D. (2010). Coherent instructional improvement and PLCs: Is it possible to do both? The Phi Delta Kappan, 91(6), 38-45.

Johnson, V. L., Simon, P., \& Mun, E.-Y. (2014). A peer-led high school transition program increases graduation rates among Latino males. The Journal of Educational Research, 107(3), 186-196. doi:10.1080/00220671.2013.788991

Johnston, W., \& Tsai, T. (2018). The Prevalence of Collaboration Among American Teachers: National Findings from the American Teacher Panel. (RAND Corporation Research Report RR-2217-BMGF). doi:10.7249/RR2217

Kallemeyn, L. M. (2014). School-level organizational routines for learning: Supporting data use. Journal of Educational Administration, 52(4), 529-548. doi:10.1108/JEA-02-2013-0025

Kassissieh, J., \& Oxley, D. (2008). From comprehensive high schools to small learning communities: accomplishments and challenges. FORUM, 50(2), 199-206. doi:10.2304/forum.2008.50.2.199

Kennelly, L., \& Monrad, M. (2007). Easing the transition to high school: Research and best practices designed to support high school learning. National High School Center. Retrieved from https://files.eric.ed.gov/fulltext/ED501073.pdf

Kowal, P. H. (2002). Vertical teaming: Making connections across levels. Middle Ground, 6(1), 20-22.

Langenkamp, A. G. (2009). Academic vulnerability and resilience during the transition to high school: The role of social relationships and district context. Sociology of Education, 83(1), 1-19. https://doi.org./10.1177/0038040709356563 
Lee, M., \& Louis, K. S. (2019). Mapping a strong school culture and linking it to sustainable school improvement. Teaching and Teacher Education, 81, 84-96. doi:10.1016/j.tate.2019.02.001

Lee, J. C., Zhang, Z., \& Yin, H. (2011). A multilevel analysis of the impact of a professional learning community, faculty trust in colleagues and collective efficacy on teacher commitment to students. Teaching and Teacher Education, 27(5), 820-830. doi:10.1016/j.tate.2011.01.006

Letrello, T. M. \& Miles, D. D. (2003). The transition from middle school to high school: Students with and without learning disabilities share their perceptions. The Clearing House, 76(4), 212.

Levi, D. (2017). Group dynamics for teams (5th ed.) Thousand Oaks, CA: Sage Publications. Levin, H., Belfield, C., Muennig, P., \& Rouse, C. (2007). The costs and benefits of an excellent education for all of America's children. Retrieved from https://www.researchgate.net/publication/216790813_The_Costs_and_Benefits_of_an_E xcellent_Education_for_America\%27s_Children

Levine, T. H., \& Marcus, A. S. (2007). Closing the achievement gap through teacher collaboration: Facilitating multiple trajectories of teacher learning. Journal of Advanced Academics, 19(1), 116-138. doi:10.4219/jaa-2007-707

Lieberman, A. (2000). Networks as learning communities: Shaping the future of teacher development. Journal of Teacher Education, 51(3), 221-227. doi:10.1177/0022487100051003010

Lindbergh High School. (2020a). Link Crew. Retrieved from https://go.lindberghschools.ws/domain/1587 
Louis, K. S., \& Murphy, J. (2017). Trust, caring and organizational learning: The leader's role. Journal of Educational Administration, 55(1), 103-126. doi:10.1108/JEA-07-2016-0077

Lovelace, M. D., Reschly, A. L., \& Appleton, J. J. (2017). Beyond school records: the value of cognitive and affective engagement in predicting dropout and on-time graduation. Professional School Counseling, 21(1). doi:10.5330/1096-2409-21.1.70

Lunenburg, F. C. (2012). Organizational structure: Mintzberg's framework. International Journal of Scholarly, Academic, and Intellectual Diversity, 14(1), 1-8.

Mac Iver, M. A., Epstein, J. L., Sheldon, S. B., \& Fonseca, E. (2015). Engaging families to support students' transition to high school: Evidence from the field. High School Journal, 99(1), 27-45. doi:10.1353/hsj.2015.0016

Madda, C. L., Halverson, R., \& Gomez, L. M. (2007). Exploring coherence as an organizational resource for carrying out reform initiatives. Teachers College Record, 109(8), 1957-1979.

Manning, K. (2013). Organizational theory in higher education. New York, NY: Routledge.

Martin, A. J. (2011). Courage in the classroom: Exploring a new framework predicting academic performance and engagement. School Psychology Quarterly, 26(2), 145-160. doi: $10.1037 / \mathrm{a} 0023020$

Masters, J. (2005). Continuity and growth: Key considerations in educational improvement and accountability. (AIRTV Seminar Series Paper No. 141). Retrieved from http://research.acer.edu.au/cgi/viewcontent.cgi?article=1009\&context=monitoring_learni ng 
Mathis, L. M. (2004). Concurrent teacher and leadership professional development in Algebra 1: Shared instructional leadership and instructional program cohesion (Doctoral dissertation, The University of Texas at Austin). Retrieved from https://repositories.lib.utexas.edu/bitstream/handle/2152/1367/mathis113335.pdf?sequenc $\mathrm{e}=2$ \&isAllowed $=\mathrm{y}$

Maynard, B. R., Salas-Wright, C. P., \& Vaughn, M. G. (2015). High school dropouts in emerging adulthood: Substance use, Mental health problems, and crime. Community Mental Health Journal, 51(3), 289-299. doi:10.1007/s10597-014-9760-5

McCallumore, K. M., \& Sparapani, E. F. (2010). The importance of the ninth grade on high school graduation rates and student success. Education Digest, 76(2), 60.

McIntosh, K., Brigid Flannery, K., Sugai, G., Braun, D. H., \& Cochrane, K. L. (2008). Relationships between academics and problem behavior in the transition from middle school to high school. Journal of Positive Behavior Interventions, 10(4), 243-255. doi:10.1177/1098300708318961

McIntosh, J., \& White, S. H. (2006). Building for freshman success: high schools working as professional learning communities. American Secondary Education, 34(2), 40.

McKee M. T., \& Caldarella P. (2016). Middle school predictors of high school performance: A case study of dropout risk indicators. Education, 136(4), 515-529.

McTighe, J., \& Emberger, M. (2006). Teamwork on assessments creates powerful professional development. Journal of Staff Development, 27(1), 38-44.

Means, B. (2010). Technology and education change: Focus on student learning. Journal of Research on Technology in Education, 42(3), 285-307. doi:10.1080/15391523.2010.10782552 
Merriam, S. B., \& Tisdell, E. J. (2015). Qualitative research: A guide to design and implementation (4th ed.). San Francisco, CA: Jossey-Bass.

Mintzberg, H. (2005). The five basic parts of the organization. In J. M. Shafritz, J. S. Ott, \& Y. S. Jang (Eds.), Classics of organizational theory (6th ed., pp. 112-124). New York, NY: Routledge,

Mintzberg, H., Quinn, J. B., \& Voyer, J. (1995). The strategy process: Collegiate edition. Upper Saddle River, NJ: Prentice-Hall.

Missouri Consultants for Education. (2010). PR 2520 Promotion and Retention. Retrieved from https://www.moconed.com/

Missouri Department of Elementary and Secondary Education (DESE). (2020a). Missouri comprehensive data system: Missouri school statistics. Retrieved from: https://apps.dese.mo.gov/MCDS/Home.aspx

Missouri Department of Elementary and Secondary Education (DESE). (2020b). Overview. Retrieved from: https://dese.mo.gov/state-board-education/overview

Missouri Department of Elementary and Secondary Education (DESE). (2020c). School improvement initiatives. Retrieved from: https://dese.mo.gov/quality-schools/schoolimprovement-initiatives

Mizelle, N. B. (2005). Moving out of middle school. Educational Leadership, 62(7), 56-60.

Mizelle, N. B., \& Irvin, J. L. (2000). Transition from middle school into high school. Middle School Journal, 31(5), 57-61. doi:10.1080/00940771.2000.11494654

Moolenaar, N. M., Sleegers, P. J. C., \& Daly, A. J. (2012). Teaming up: Linking collaboration networks, collective efficacy, and student achievement. Teaching and Teacher Education, 28(2), 251-262. doi:10.1016/j.tate.2011.10.001 
Moore, J. L., Way, J. D., Casillas, A., Burrus, J., Allen, J., \& Hanson, M. A. (2016). Effects of psychosocial characteristics of middle school students on high school grades and on-time graduation. European Journal of Psychological Assessment, 32(1), 75-83. doi:10.1027/1015-5759/a000334

Morgan, P. L., \& Hertzog, C. J. (2001). Designing comprehensive transitions. Principal Leadership: Middle Level Edition, 1(7), 10-16.

National Center for Education Statistics. (2018). Trends in high school dropout and completion rates in the United States. Retrieved from https://nces.ed.gov/programs/dropout/intro.asp

National Center for Education Statistics. (2020). Trends in high school dropout and completion rates in the United States. Retrieved from https://nces.ed.gov/programs/dropout/intro.asp

Neild, R. C., (2009). Falling off track during the transition to high school: What we know and what can be done. The Future of Children, 19(1), 53-76. doi:10.1353/foc.0.0020

Neild, R. C., Balfanz, R., \& Herzog, L. (2007). An early warning system. Educational Leadership, 65(2), 28.

Neild, R. C., Stoner-Eby, S., \& Furstenberg, F. (2008). Connecting entrance and departure: The transition to ninth grade and high school dropout. Education and Urban Society, 40(5), 543-569. doi:10.1177/0013124508316438

Nelson, H. K., Fairchild, M., Grossenbacher, M., \& Landers, L. (2007). Examining effective middle grades programs: Stating implications for secondary school reform. American Secondary Education, 35(2), 52-68.

Newman, B. M., Newman, P. R., Griffen, S., O’Connor, K., \& Spas, J. (2007). The relationship of social support to depressive symptoms during the transition to high school. Adolescence, 42(167), 441-459. 
Newmann, F. M., Smith, B., Allensworth, E., \& Bryk, A. S. (2001a). Instructional program coherence: What it is and why it should guide school improvement policy. Educational Evaluation and Policy Analysis, 23(4), 297-321. doi:10.3102/01623737023004297

Newmann, F. M., Smith, B., Allensworth, E., \& Byrk, A. S. (2001b). School instructional program coherence: Benefits and challenges (Descriptive Report (141) ED 451 305). Consortium on Chicago School Research. Retrieved from https://files.eric.ed.gov/fulltext/ED451305.pdf

Nicholson, S. A., \& Bond, N. (2003). Collaborative reflection and professional community building: an analysis of preservice teachers' use of an electronic discussion board. Journal of Technology and Teacher Education, 11(2), 259-279.

Northouse, P. G. (2016). Leadership: Theory and practice. Thousand Oaks, CA: Sage. Office of Vocational and Adult Education (OVAE). (2003). The road to reform of American high school (ED-99-CO-0163). U.S. Department of Education. Retrieved from https://www2.ed.gov/about/offices/list/ovae/pi/hsinit/papers/history.pdf

Ohlsson, J. (2013). Team learning: Collective reflection processes in teacher teams. Journal of Workplace Learning, 25(5), 296-309. doi:10.1108/JWL-Feb-2012-0011

Oakes, A. (2009) Middle-to-high-school transition. Retrieved from: https://files.eric.ed.gov/fulltext/ED506363.pdf

Oakes, A., \& Waite, W. (2009, May). Middle-to-high-school transition: Practical strategies to consider (Learning Point Associates Newsletter). The Center for Comprehensive School Reform and Improvement. Retrieved from https://files.eric.ed.gov/fulltext/ED506363.pdf 
Parrish, T., Poland, L., Arellanes, M., Ernandes, J., \& Viloria, J. (2011). Making the move: Transition strategies at California schools with high graduation rates: (537252012-001) [Data set]. American Psychological Association. doi:10.1037/e537252012-001

Pharris-Ciurej, N., Hirschman, C., \& Willhoft, J. (2012). The 9th grade shock and the high school dropout crisis. Social Science Research, 41(3), 709-730. doi:10.1016/j.ssresearch.2011.11.014

Prenger, R., Poortman, C. L., \& Handelzalts, A. (2019). The effects of networked professional learning communities. Journal of Teacher Education, 70(5), 441-452. doi: $10.1177 / 0022487117753574$

Reents, J. N. (2002). Isolating 9th graders. School Administrator, 59(3), 14-19.

Ronfeldt, M., Farmer, S., McQueen, K., \& Grissom, J. (2015). Teacher collaboration in instructional teams and student achievement. American Educational Research Journal, $52(3), 475-514$.

Rosen, J. A., Warkentien, S., \& Rotermund, S. (2019). Stopping out versus dropping out: The role of educational resilience in explaining on-time completion of high school. American Journal of Education, 125(2), 259-287. doi:10.1086/701248

Roybal, V., Thornton, B., \& Usinger, J. (2014). Effective ninth-grade transition programs can promote student success. Education, 134(4), 475-487.

Rumberger, R. W. (2013). Poverty and high school dropouts. American Psychological Association, 34(10).

Sage Publishing. (2020). NASSP bulletin. Retrieved from https://us.sagepub.com/enus/nam/journal/nassp-bulletin\#description 
Saint John Vianney High School. (2020). Link Crew. Retrieved from https://www.vianney.com/link-crew.html

Şahin, F., \& Cemaloğlu, N. (2015). In-school variables supporting and inhibiting organizational learning in secondary education institutions. Procedia - Social and Behavioral Sciences, 174, 2912-2919. doi:10.1016/j.sbspro.2015.01.1028

Schein, E. H. (2005). Defining organizational culture. In Shafritz J., Ott J., \& Yang, Y. (Eds.) Classics of organization theory, (6th ed., pp. 360-367). Belmont, CA: Wadsworth.

Schlosser, L. (2015). Transition by design: The power of vertical teams. AMLE Magazine 2(8), 18-20. Retrieved from https://fisherpub.sjfc.edu/education_facpub/32

Schmitt, A., Raisch, S., \& Volberda, H. W. (2018). Strategic renewal: Past research, theoretical tensions and future challenges. International Journal of Management Reviews, 20(1), 8198. doi:10.1111/ijmr.12117

Schulz, K.-P., \& Geithner, S. (2010). Individual and organizational development as interplay: An activity-oriented approach. German Journal of Human Resource Management, 24(2), 130-151. doi:10.1177/239700221002400203

Sedalia School District 200. (2020). Smith-Cotton Freshman Academy. Retrieved from https://www.sedalia200.org/Page/999

Seidman, I. (2013). Interviewing as qualitative research. (4th ed.). New York, NY: Teachers College Press.

Sekulich, K. M. (2019). Curriculum and assessment alignment mapping. Lutheran Education, 155(2), 33-39. 
Silverthorn, N., Dubois, D. L., \& Crombie, G. (2005). Self-perceptions of ability and achievement across the high school transition: Investigation of a state - trait model. The Journal of Experimental Education, 73(3), 191-218. doi:10.3200/JEXE.73.3.191-218

Slavin, R. E., Lake, C., \& Groff, C. (2009). Effective programs in middle and high school mathematics: A best-evidence synthesis. Review of Educational Research, 79(2), 839911. doi: $10.3102 / 0034654308330968$

Slick, S. (2002). Teachers are enthusiastic participants in a learning community. The Clearing House: A Journal of Educational Strategies, Issues and Ideas, 75(4), 198-201. doi:10.1080/00098650209604931

Smith, J. S. (2006a). Examining the long-term impact of achievement loss during the transition to high school. Journal of Secondary Gifted Education, 17(4), 211-221.

Smith, J. S. (2006b). Similarities and differences in students' and parents' perceptions of the transition from middle school to high school. Research in Middle Level Education Online, 29(10), 1-9. doi:10.1080/19404476.2006.11462033.

Smith, T. J. (2007). Managing the transition to ninth grade in a comprehensive urban high school. (National High School Center Report). Retrieved from https://files.eric.ed.gov/fulltext/ED501072.pdf

Smith, J. S., Akos, P., Lim, S., \& Wiley, S. (2008). Student and stakeholder perceptions of the transition to high school. The High School Journal, 91(3), 32-42. doi:10.1353/hsj.2008.0003

Somers, C. L., Owens, D., \& Piliawsky, M. (2009). A study of high school dropout prevention and at-risk ninth graders' role models and motivations for school completion. Education, 130(2), 348-356. 
Sorensen, L. C. (2019). "Big data" in educational administration: An application for predicting school dropout risk. Educational Administration Quarterly, 55(3), 404-446. doi:10.1177/0013161X18799439

Springfield Public Schools. (n.d.). SPS to launch freshman pilot course this fall at GHS and HHS. Retrieved from: https://www.sps.org/site/default.aspx?PageType=3\&DomainID=4\&ModuleInstanceID=1 305\&ViewID=6446EE88-D30C-497E-93163F8874B3E108\&RenderLoc $=0 \&$ FlexDataID $=3607 \&$ PageID $=1$

Squires, D. (2012). Curriculum alignment research suggests that alignment can improve student achievement. Clearing House, 85(4), 129-135. doi:10.1080/00098655.2012.657723

Statista. (n.d.). Average composite American college testing (ACT) score in the United States in 2019, by state. Retrieved from https://www.statista.com/statistics/305987/us-average-actscores-by-state/

Stosich, E. L., Bocala, C., \& Forman, M. (2018). Building coherence for instructional improvement through professional development: A design-based implementation research study. Educational Management Administration \& Leadership, 46(5), 864-880.

Suh, J., \& Seshaiyer, P. (2015). Examining teachers' understanding of the mathematical learning progression through vertical articulation during lesson study. Journal of Mathematics Teacher Education, 18(3), 207-229. doi:10.1007/s10857-014-9282-7

Suldo, S. M., \& Shaunessy-Dedrick, E. (2013). Changes in stress and psychological adjustment during the transition to high school among freshmen in an accelerated curriculum. Journal of Advanced Academics, 24(3), 195-218. doi:10.1177/1932202X13496090 
Suldo, S. M., Shaunessy, E., Thalji, A., Michalowski, J., \& Shaffer, E. (2009). Sources of stress for students in high school college preparatory and general education programs: Group differences and associations with adjustment. Adolescence, 176, 925.

Sutton, A., Langenkamp, A. G., Muller, C., \& Schiller, K. S. (2018). Who gets ahead and who falls behind during the transition to high school? Academic performance at the intersection of race/ethnicity and gender. Social Problems, 65(2), 154-173. doi:10.1093/socpro/spx044

Taylor \& Francis Online. (2020). The clearing house: A journal of educational strategies, issues and ideas. Retrieved from https://www.tandfonline.com/action/journalInformation?show=aimsScope\&journalCode $=\operatorname{vtch} 20$

Timberland High School. (2020). Link Crew. Retrieved from https://www.wentzville.k12.mo.us/Page/7120

Thattai, D. (2017). A History of Public Education in the United States Editorial Summary. Retrieved from https://www.researchgate.net/publication/321179948_A_History_of_Public_Education_i n_the_United_States_Editorial_Summary

Thayer, A. J., Cook, C. R., Fiat, A. E., Bartlett-Chase, M. N., \& Kember, J. M. (2018). Wise feedback as a timely intervention for at-risk students transitioning into high school. School Psychology Review, 47(3), 275-290. doi:10.17105/SPR-2017-0021.V47-3 Theoharis, G. (2008). Woven in Deeply: Identity and Leadership of Urban Social Justice Principals. Education and Urban Society, 41(1), 3-25. https://doi.org/10.1177/0013124508321372 
University Council for Educational Administration (UCEA). (2020). Journal of research on leadership education. Retrieved from https://journals.sagepub.com/home/jrl

Useem, E., Nei, R. C., \& Morrison, W. (2001). Philadelphia's talent development high schools: Second-year results, 2000-0 (Report - Research (143) ED 467 132). Philadelphia Education Fund. Retrieved from https://files.eric.ed.gov/fulltext/ED467132.pdf

Uvaas, T., \& McKevitt, B. C. (2013). Improving transitions to high school: A review of current research and practice. Preventing School Failure, 57(2), 70-76. doi:10.1080/1045988X.2012.664580

Vangrieken, K., Dochy, F., Raes, E., \& Kyndt, E. (2015). Teacher collaboration: A systematic review. Educational Research Review, 15, 17-40. doi:10.1016/j.edurev.2015.04.002

Vera, E., Shriberg, D., Alves, A., Montes de Oca, J., Reker, K., Roche, M., Salgado, M., Stegmaier, J., Viellieu, L., Karahalios, V., Knoll, M., Adams, K., Diaz, Y., \& Rau, E. (2016). Evaluating the impact of a summer dropout prevention program for incoming freshmen attending an under-resourced high school. Preventing School Failure, 60(2), 161-171. doi:10.1080/1045988X.2015.1063039

Western, B., \& Pettit, B. (2002). Beyond crime and punishment: Prisons and inequality. Contexts, 1(3), 37-43. doi:10.1525/ctx.2002.1.3.37

Wentzel, K. R., Tomback, R., Williams, A., \& McNeish, D. (2019). Perceptions of competence, control, and belongingness over the transition to high school: A mixed-method study. Contemporary Educational Psychology, 56, 55-66. doi:10.1016/j.cedpsych.2018.11.005

Youngman, M. B. (1986). Editorial review. In M. B.Youngman (Ed.), Mid-schooling transfer: Problems and proposals (pp. 137-140). Philadelphia, PA: NFER-Nelson. 
Youngs, P., Holdgreve-Resendez, R. T., \& Qian, H. (2011). The role of instructional program coherence in beginning elementary teachers' induction experiences. The Elementary School Journal, 111(3), 455-476. doi:10.1086/657655

Zettelmeyer, F. (2015). A Leader's Guide to Data Analytics. Kellogg Insight. Retrieved from https://insight.kellogg.northwestern.edu/article/a-leaders-guide-to-data-analytics

Zettelmeyer, F., \& Bolling, M. (2014). Big data doesn't make decisions, leaders do. [Report Kellogg Initiatives: Markets and Customers]. Kellogg School of Management, Nortwestern University. Retrieved from https://insight.kellogg.northwestern.edu/article/aleaders-guide-to-data-analytics 
Appendix

\section{Updated Survey}

\section{Informed Consent}

Greetings,

My name is Kyle Thompson and I am a doctoral candidate at the University of Missouri in cooperation with Missouri State University. I am conducting a research study to examine the relationship between Instructional Program Coherence of high school and feeder school teachers with student achievement from the high school principal perspective. The transition to high school can cause a unique disruption that affects student achievement and consequently students' ability to graduate. School districts are allocating significant effort and resources in response, to ease this transition for students. Your participation in this study will be beneficial in determining if and how teacher collaboration and coherence can further ease the academic transition for students making the transition.

As a part of this study, I ask that you take part in a brief three-section survey consisting of 35 items (29 multiple choice and 6 numerical). The survey should take no more than 30 minutes to complete. The first section will consist of three items to see if you qualify and consent to participate in the study. If you do not, you will be redirected to exit the survey. It is important to note that due to Covid-19, this study has been adjusted to seek data from the 2018-2019 school year. The questions and participation have been adjusted accordingly.

Your participation in this study is voluntary and you can withdraw at any time without penalty. You will not be penalized for skipping questions, refusal to respond, or withdrawal from the study. Your participation in this study will also be confidential and all email addresses and data will be kept securely and only seen by the researcher. The risk of this study is minor. By completing the survey, you acknowledge that your participation in the study is voluntary, you are 18 years of age, and you are aware that you may choose to terminate your participation in the study at any time and for any reason.

If you have any questions, please contact Kyle Thompson at (715)-340-5651 or thompsonkj24@gmail.com. You can also contact the supervising advisor for this project: Dr. Kim Finch, (417)-836-5192, KimFinch@MissouriState.edu. If you want to talk privately about your rights or any issues related to your participation in this study, you can contact the University of Missouri Research Participant Advocacy by calling 888-280-5002 (a free call) or emailing MUResearchRPA@ missouri.edu. Thank you for your time. I appreciate your participation. 
Sincerely,

Kyle J. Thompson

University of Missouri/Missouri-State University

Educational Leadership and Policy Analysis

*** Only the survey question portions on the following pages need to be mailed back in the self-addressed stamped envelope. You can keep or discard this letter. 
Survey Questions - Return this portion in the self-addressed stamped envelope. In case the envelope is lost, return to the following address:

\section{Kyle J. Thompson}

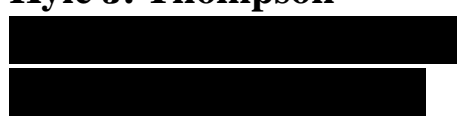

Survey \#

\section{Section 1: Qualifying Questions}

1. How many years have you held an administrative position (principal or assistant principal) at your current high school?

\section{Section 2: Freshmen Data}

2. Due to Covid-19, this study requires freshmen data from the $2018-2019$ school year. If there is a record keeper or staff member within the district that can better provide the freshmen data from the 2018-2019 school year, please provide their name and contact information below and you may skip to the Section 3 of the survey. If you have access to these records, please complete the rest of this section.

Name:

Title:

Email:

Phone:

3. Approximately how many of your students currently qualify as freshmen by credit accumulation requirements?

4. To the nearest tenth of a percent, what was your freshman course failure rate in the 20182019 school year?

5. To the nearest tenth of a percent, what was your freshman course completion rate for the 2018-2019 school year?

6. What was your average freshman grade point average for the 2018-2019 school year?

7. To the nearest tenth of a percent, what was your freshman attendance rate in the 20182019 school year?

8. To the nearest tenth of a percent, what was the rate of freshmen who received at least one out-of-school suspension in the 2018-2019 school year? 
Section 3: Instructional Program Coherence - For each of the following topic areas, please circle the number of the phrase that best captures the activity of teachers in your building.

9. Teachers within disciplines purposely link their curriculum to learning goals.

1 Completely Absent $=$ Teachers within disciplines do not connect their curriculum to these goals.

2 Slightly Present $=$ Some teachers within disciplines connect some of their curriculum to these goals.

3 Mostly Present $=$ All teachers within disciplines connect some of their curriculum to these goals.

4 Completely Present $=$ Teachers within disciplines connect their entire curriculum to these goals.

\section{Teachers within disciplines use common instructional strategies.}

1 Completely Absent $=$ Teachers within disciplines do not use common instructional strategies.

2 Slightly Present $=$ Some teachers within disciplines use some common instructional strategies.

3 Mostly Present $=$ All teachers within disciplines use some common instructional strategies.

4 Completely Present $=$ All teachers within disciplines use many common instructional strategies.

\section{Teachers within disciplines use common assessment strategies.}

1 Completely Absent $=$ Teachers within disciplines do not use common assessment strategies.

2 Slightly Present $=$ Some teachers within disciplines use some common assessment strategies.

3 Mostly Present $=$ All teachers within disciplines use some common assessment strategies.

4 Completely Present $=$ All teachers within disciplines use many common assessment strategies. 
12. Teachers coordinate curriculum to avoid repetition and to offer students new and more complex aspects of subject matter as they move from grade to grade.

1 Completely Absent $=$ Teachers do not coordinate curriculum across the grades .

2 Slightly Absent $=$ Some teachers coordinate some curriculum across the grades.

3 Mostly Present $=$ All teachers coordinate some curriculum across the grades.

4 Completely Present $=$ All teachers coordinate all curriculum across the grades .

13. Teachers coordinate assessments to avoid repetition and to offer students new and more complex aspects of subject matter as they move from grade to grade.

1 Completely Absent $=$ Teachers do not coordinate assessments across the grades .

2 Completely Absent $=$ Some teachers coordinate some assessments across the grades .

3 Mostly Present $=$ All teachers coordinate some assessments across the grades .

4 Completely Present $=$ All teachers coordinate many assessments across the grades .

\section{School-sponsored support programs, such as remedial instruction, field trips,} tutoring, and parent supports, are linked to the curriculum, instruction, and assessments of the school program.

$1=$ Few to no support programs are linked to the curriculum, instruction, and assessments of the school program.

2 = Some support programs are linked to the curriculum, instruction, and assessments of the school program.

$3=$ Most support programs are linked to the curriculum, instruction, and assessments of the school program.

$4=$ Almost all support programs are linked to the curriculum, instruction, and assessments of the school program. 


\section{Professional development for staff supports the implementation of common curriculum.}

$1=$ Professional development for staff does not support the implementation of common curriculum.

$2=$ Professional development for staff only weakly or sporadically supports the implementation of common curriculum.

$3=$ Professional development for staff regularly supports the implementation of common curriculum.

$4=$ Professional development for staff extensively supports the implementation of common curriculum.

\section{Professional development for staff supports the implementation of common instructional strategies.}

$1=$ Professional development for staff does not support the implementation of common instructional strategies.

$2=$ Professional development for staff only weakly or sporadically supports the implementation of common instructional strategies.

$3=$ Professional development for staff regularly supports the implementation of common instructional strategies.

$4=$ Professional development for staff extensively supports the implementation of common instructional strategies.

\section{Professional development for staff supports the implementation of common assessments.}

$1=$ Professional development for staff does not support the implementation of common assessments.

$2=$ Professional development for staff only weakly or sporadically supports the implementation of common assessments.

$3=$ Professional development for staff regularly supports the implementation of common assessments.

$4=$ Professional development for staff extensively supports the implementation of common assessments. 


\section{Professional development programs are sustained over time.}

$1=$ No professional development programs (or almost none) are sustained over time.

$2=$ Some professional development programs are sustained over time.

3 = Many professional development programs are sustained over time.

$4=$ Almost all professional development programs are sustained over time.

\section{The school strategically accepts and refuses programs and initiatives in a manner that supports staff focus, program continuity, and ongoing improvement.}

$1=$ The school has many programs, and staff energy and attention are very fragmented. Thus, the programs do not support staff focus, program continuity, or ongoing improvement.

$2=$ The school has many programs, but some attempts are made to focus staff energies and attention to support program continuity and ongoing improvement.

$3=$ The school is somewhat strategic in how it accepts and refuses programs so that they may support staff focus, program continuity, and ongoing improvement.

$4=$ The school is very strategic in how it accepts and refuses programs so that they may support staff focus, program continuity, and ongoing improvement.

\section{School improvement planning and assessment directly address the school's progress in providing a common, coordinated, and sustained school program.}

$1=$ School improvement planning and assessment generate long, disconnected lists of goals and assessments.

$2=$ Some school improvement planning and assessment addresses the school's progress in providing a common, coordinated, and sustained school program.

$3=$ Much school improvement planning and assessment addresses the school's progress in providing a common, coordinated, and sustained school program.

$4=$ A great deal of school improvement planning and assessment addresses the school's progress in providing a common, coordinated, and sustained school program. 
21. Curriculum remains reasonably stable over time and thus provides teachers with sustained opportunities to learn how to teach it well.

$1=$ Curriculum is very unstable and frequently changes. Teachers have no sustained opportunity to learn how to teach it well.

$2=$ Curriculum is only partially stable over time. Teachers have sustained opportunities to learn how to teach some of it well.

$3=$ Curriculum is fairly stable over time. Teachers have sustained opportunities to learn how to teach much of it well.

$4=$ Curriculum is very stable over time. Teachers have sustained opportunities to learn how to teach most of it well.

\section{Assessment remains reasonably stable over time and thus provides teachers with} sustained opportunities to teach students how to succeed on them.

$1=$ Assessment is very unstable and frequently changes. Teachers have no sustained opportunity to teach students how to succeed on them.

$2=$ Assessment is only partially stable over time. Teachers have sustained opportunities to teach students how to succeed on some of them.

$3=$ Assessment is fairly stable over time. Teachers have sustained opportunities to teach students how to succeed on many of them.

$4=$ Assessment is very stable over time. Teachers have sustained opportunities to teach students how to succeed on most of them.

\section{Instruction remains reasonably stable over time and thus provides teachers with sustained opportunities to focus and improve their practices.}

$1=$ Instruction is very unstable and frequently changes. Teachers have no sustained opportunity to focus and improve their practices.

$2=$ Instruction is only partially stable over time. Teachers have sustained opportunities to focus and improve their practices.

$3=$ Instruction is fairly stable over time. Teachers have sustained opportunities to focus and improve their practices.

$4=$ Instruction is very stable over time. Teachers have sustained opportunities to focus and improve their practices. 


\section{Teaching assignments remain stable over time.}

$1=$ Teaching assignments are very unstable and frequently change.

$2=$ Some teaching assignments are stable while others frequently change.

3 = Many teaching assignments are stable over time.

$4=$ Most teaching assignments remain stable over time.

\section{Key program leaders or leadership positions remain stable over time.}

$1=$ Key program leaders or leadership positions are very unstable over time and frequently change.

$2=$ Some key program leaders or leadership positions are stable while others frequently change.

3 = Many key program leaders or leadership positions are stable over time.

$4=$ Most key program leaders or leadership positions are stable over time and rarely change.

\section{Teachers implementation in classroom practices are consistent throughout the school and adhere to a common set of guidelines.}

$1=$ Teachers do not coordinate technology practices and rarely adhere to technology guidelines.

$2=$ Teachers do not coordinate technology practices but do adhere to technology guidelines.

$3=$ Some teachers coordinate technology practices and adhere to technology guidelines.

$4=$ All teachers coordinate practices vertically and horizontally and universally adhere to technology guidelines. 
27. Teachers coordinate consistent classroom rules, expectations, protocols, and policies that are appropriate to the grade level.

$1=$ Teachers do not coordinate classroom rules, expectations, protocols, and policies.

$2=$ Teachers coordinate some classroom rules, expectations, protocols, and policies that are appropriate to grade levels.

$3=$ Grade level teachers or teachers within a discipline coordinate consistent classroom rules, expectations, protocols, and policies that are appropriate to the grade level.

4 = Appropriate classroom rules, expectations, protocols, and policies are coordinated universally throughout the school, both vertically and horizontally, and between disciplines.

28. Teachers maintain high and appropriate academic and classroom standards for their students that properly prepare them for success in future courses.

$1=$ There is little or no coordination of academic and classroom standards between grade levels.

$2=$ Some teachers coordinate academic and classroom standards between grade levels but only some prepare their students properly.

$3=$ All teachers within a discipline coordinate academic and classroom standards between grade levels and most prepare their students properly.

$4=$ All teachers within a discipline coordinate academic and classroom standards between grade levels and continuously communicate results to strive to achieve proper preparation for all students.

\section{Teachers within a discipline collaborate to collect, disseminate, and analyze student data to inform teaching practices.}

$1=$ There is little or no data usage collaborated on by teachers.

$2=$ Teachers within a discipline collaborate to collect and disseminate data, but do not use it to inform teaching practices.

3 = All teachers within a discipline collect, disseminate, analyze data but only some independently use it to inform teaching practices.

$4=$ All teachers within a discipline collect, disseminate, analyze data and most or all collaborate to use that information to improve teaching practices. 
30. Technology and training in technology is accessible to teachers and results in technological practices that increase engaging instruction.

$1=$ Technology or technology training is not accessible enough for teachers to utilize in their work.

$2=$ Only some teachers actively participate in technology training and utilize it to increase engaging instruction.

$3=$ Most teachers actively participate in technology training and utilize it to increase engaging instruction.

$4=$ All teachers actively participate in technology training and utilize it to increase engaging instruction. They also share expertise to help others improve technology-based instruction.

\section{Technology and training in technology is accessible to teachers and results in technological practices that increase efficiency and effectiveness of collaboration between teachers of different grade levels.}

$1=$ Technology or technology training is not accessible enough for teachers to utilize in their collaboration.

$2=$ Only some teachers actively participate in technology training and utilize it to increase efficiency and effectiveness of their collaboration.

$3=$ Most teachers actively participate in technology training and utilize it to increase efficiency and effectiveness of their collaboration.

$4=$ All teachers actively participate in technology training and utilize it to increase efficiency and effectiveness of their collaboration. They also share expertise and help others improve their collaboration through technology use. 
32. Teachers within a discipline collaborate vertically to collect, disseminate, and analyze student data to inform implementation of proper interventions for at-risk students.

$1=$ There is little or no data usage collaborated on by teachers.

$2=$ Teachers within a discipline collaborate to collect and disseminate data, but do not use it to implement proper interventions for at-risk students.

3 = All teachers within a discipline collect, disseminate, analyze data but only some independently use it to implement proper interventions for at-risk students.

$4=$ All teachers within a discipline collect, disseminate, analyze data and most or all collaborate to use that information to implement proper interventions for at-risk students.

\section{High school and mid-level feeder school teachers participate in formal collaboration} to communicate and align the in-class expectations and rigor of classes surrounding the transition to high school.

$1=$ There is little or no formal collaboration between mid-level feeder school and high school teachers.

$2=$ Mid-level feeder school and high school teachers occasionally collaborate to communicate and align the in-class expectations and rigor of classes surrounding the transition to high school.

$3=$ Mid-level feeder school and high school teachers often collaborate to communicate and align the in-class expectations and rigor of classes surrounding the transition to high school.

$4=$ Mid-level feeder school and high school teachers consistently collaborate to communicate and align the in-class expectations and rigor of classes surrounding the transition to high school and use student achievement data to guide change in preparation. 
34. Opportunities have been created within the school structure for consistent vertical collaboration between high school teachers.

$1=$ There are few or no built-in opportunities for vertical collaboration to occur.

$2=$ Teachers have designated opportunities for formal vertical collaboration during school hours monthly throughout the school year.

$3=$ Teachers have designated opportunities for vertical collaboration during school hours multiple times a month throughout the school year.

$4=$ Schedules contain common planning time for teachers of different grade levels within the same discipline or designated opportunities for vertical collaboration occur at a minimum of once a week.

\section{The culture at our high school promotes consistent effective vertical collaboration between teachers.}

$1=$ Our culture is not currently conducive to consistent vertical collaboration between teachers.

$2=$ Some vertical collaboration occurs, but we are taking the initiative to make our culture more conducive for effective vertical collaboration to take place consistently between teachers.

$3=$ Effective vertical collaboration occurs often between our high school teachers and is engrained into our building's culture.

4 = Effective vertical collaboration is engrained into our school's culture and occurs consistently between teachers within the building, but also between our high school teachers and teachers from the mid-level feeder schools to a comfortable degree.

Thank you for completing this survey! Your participation is greatly appreciated. 


\section{VITA}

Kyle James Thompson was born in Stevens Point, Wisconsin. He graduated from TriCounty High School in Plainfield, Wisconsin in 2001. Upon graduation, he attended the University of Wisconsin-Whitewater in Whitewater, Wisconsin in 2006 with a bachelor's degree in Secondary Mathematics Education and a Coaching minor. He went on to teach secondary mathematics spanning grades 7 through 12 for multiple school districts in the central Wisconsin area from the 2006 to 2015. During that span graduated with a master's degree in Technology Education from Marian University in Fon du lac, Wisconsin in 2011. His final teaching position in Wisconsin was for Oshkosh West High School in Oshkosh, Wisconsin before he moved to Missouri in 2015.

In the fall of 2015, Kyle obtained a position as a secondary mathematics educator at Glendale High School in Springfield, MO. The focus for his last two positions has been on the freshman grade level. He helped implement and has been a teaching member the Freshman Academy at Glendale High School since its inception in 2018. He enrolled at the University of Missouri in 2017 as a part of the Missouri State cohort in a cooperative statewide program. He graduated from the University of Missouri with a doctoral degree in Educational Leadership and Policy Analysis in May 2021. 